\title{
In-situ investigation of vapor-deposited glasses of toluene and ethylbenzene via alternating current chip-nanocalorimetry
}

\author{
Dissertation \\ zur \\ Erlangung des akademischen Grades \\ doctor rerum naturalium (Dr. rer. nat.) \\ der Mathematisch-Naturwissenschaftlichen Fakultät \\ der Universität Rostock

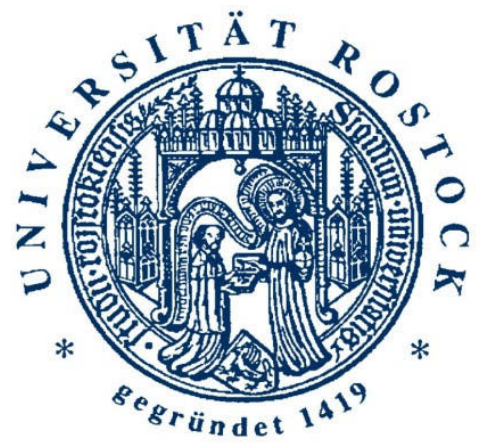 \\ vorgelegt von \\ Dipl.-Phys. Mathias Ahrenberg \\ aus Roggentin \\ geb. am 22.11.1980 in Schwerin
}

Rostock, Juli 2013

urn: nbn: de :gbv:28-diss2013-0123-4 


\section{Gutachter:}

1. Gutachter:

Prof. Dr. Christoph Schick

Institut für Physik, Universität Rostock

2. Gutachter

Prof. Dr. Mark Ediger

Department of Chemistry, University of Wisconsin

3. Gutachter

Prof. Dr. Mario Beiner

Institut für Chemie, Universität Halle

Datum der Einreichung: $\quad$ 26.02.2013

Datum der Verteidigung: $\quad$ 29.05.2013 


\section{Table of Contents}

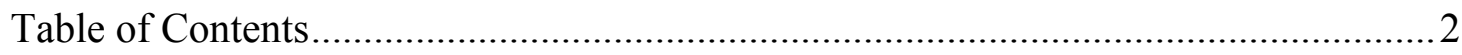

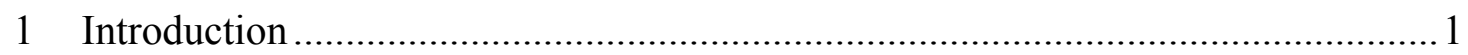

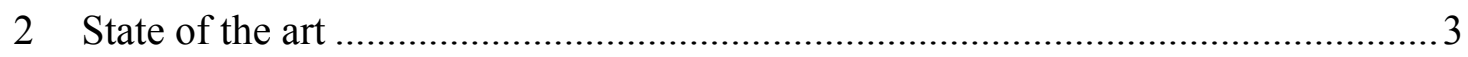

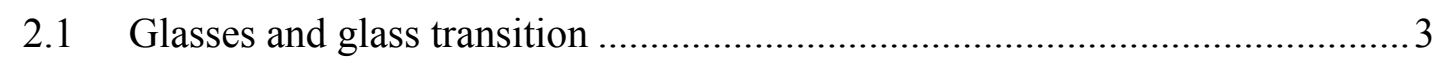

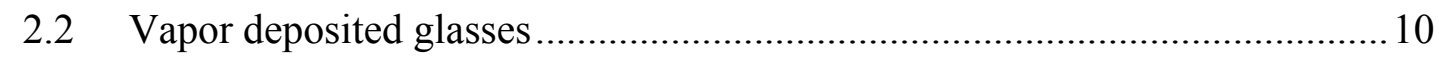

2.2.1 Two different observations of the stability of vapor-deposited glasses ....10

2.2.2 Possible mechanism of stable glass formation .................................... 13

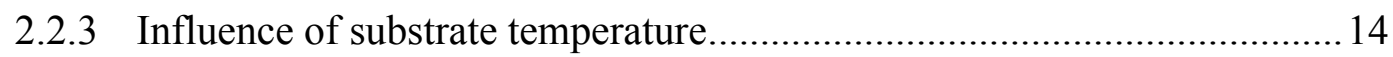

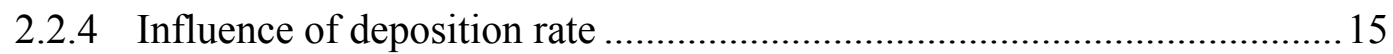

2.2.5 Transformation of a stable glass to the super cooled liquid .....................16

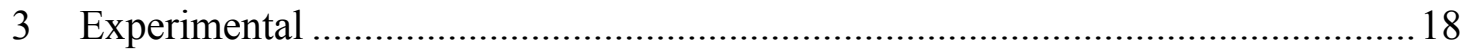

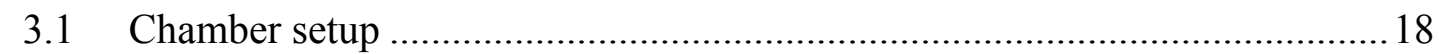

3.1.1 Requirements for in-situ AC calorimetry of vapor-deposited samples ..... 18

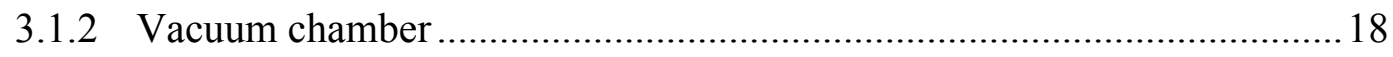

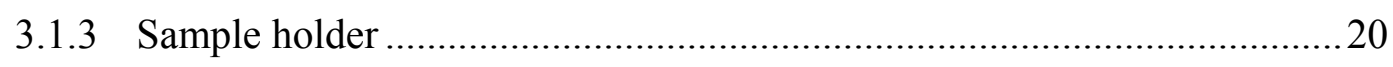

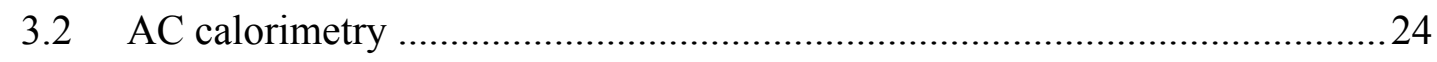

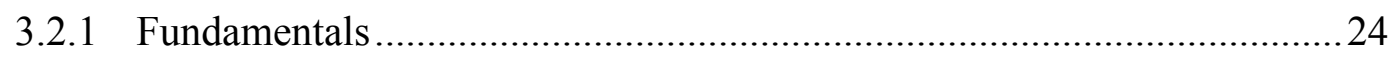

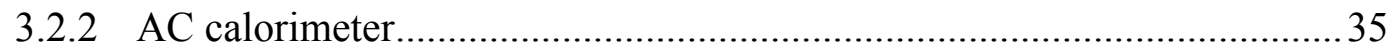

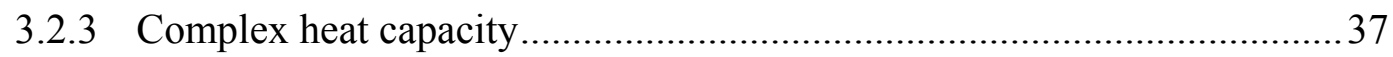

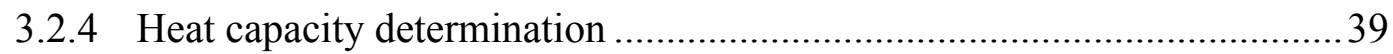

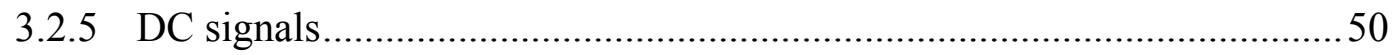

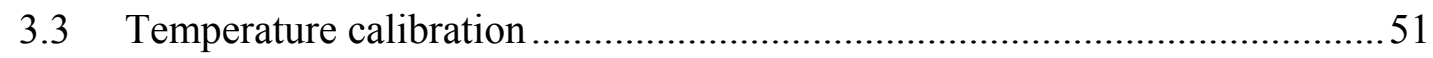

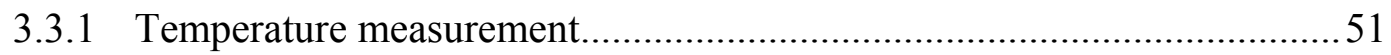

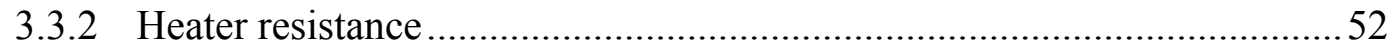

3.3.3 Temperature calibration of the heater resistance ...................................54

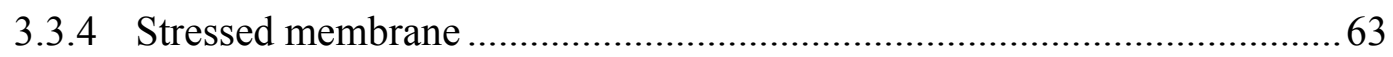

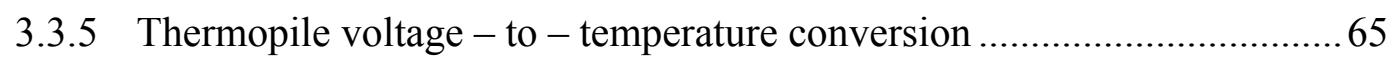




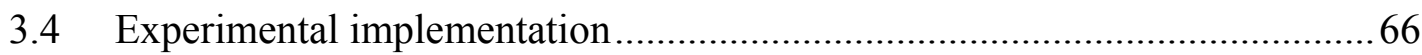

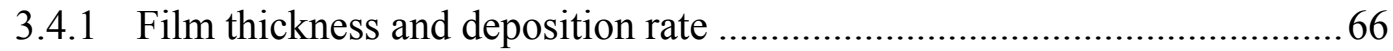

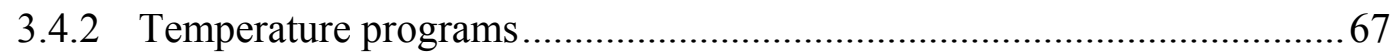

4 In-situ measurements of vapor-deposited toluene and ethylbenzene .....................69

4.1 Detection limit for heat capacity and a possible mobile layer ......................69

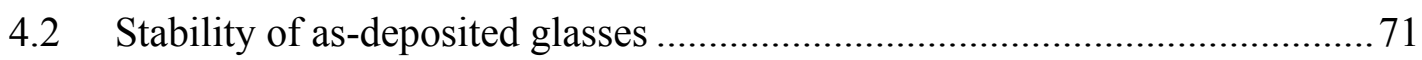

4.3 Stability depending on substrate temperature .............................................. 75

4.4 Stability depending on deposition rate .................................................. 77

4.5 Transformation time dependence on temperature and film thickness ............ 79

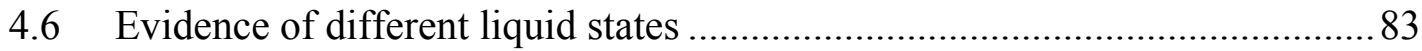

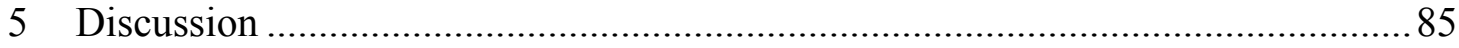

5.1 Influence of substrate temperature and deposition rate on heat capacity ....... 85

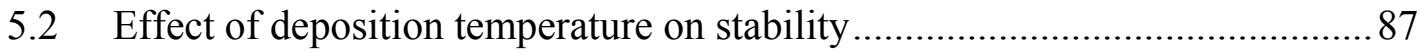

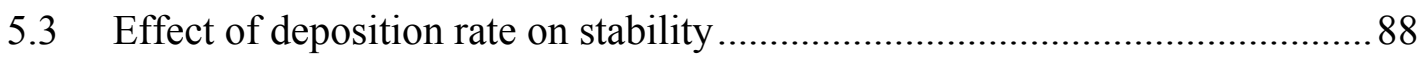

5.4 Transformation time vs. structural relaxation time................................... 90

5.5 Influence of deposition temperature on the liquid state.............................. 91

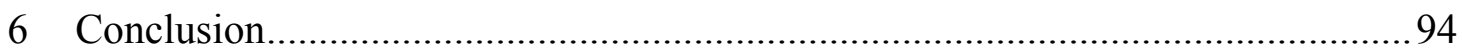

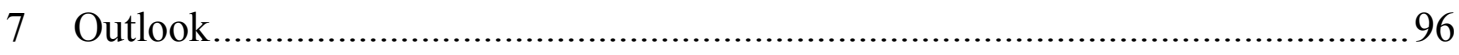

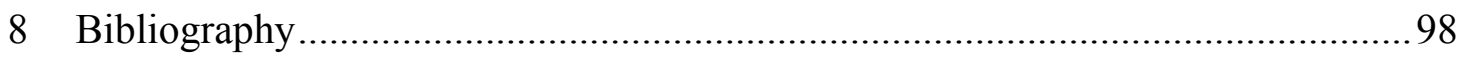

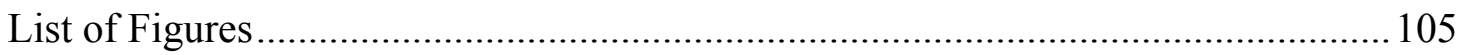

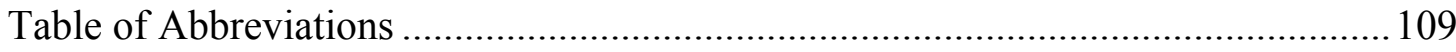

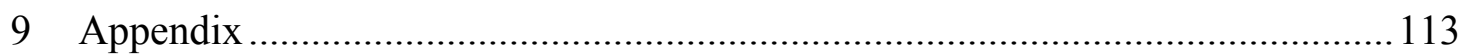


Glasses have been produced and used by mankind for thousands of years but understanding the main principles behind glass formation and structure is still an issue of present investigations. Since ancient times $\mathrm{SiO}_{2}$ was used for making glasses; nowadays the variety of glass-forming materials is wide-spread. Glasses can be obtained from organic, inorganic, polymeric, colloidal and even metallic components [1-3]. A glass is commonly formed by cooling a liquid from the melt into the supercooled liquid state (SCL). Further cooling causes the molecular motions in the melt to slow down until the amorphous structure "freezes-in" yielding a solid. Thus glasses are amorphous solids. The temperature of the freezing-in of a glass former is denoted as the glass transition temperature $T_{g}$ [4]. Thermodynamically equilibrium is lost at $T_{g}$ since molecular rearrangements becomes so slow that equilibration during further cooling is impossible. This implies the glass transition temperature is a function of the cooling rate (see Figure 1).

The non-equilibrium glass represents a thermodynamically unstable state. These systems seek equilibrium and consequently glasses continuously relax until equilibrium is reached. Isothermal relaxation is denoted as aging, but even a few ten Kelvin below $T_{g}$ full equilibration will never be reached on the experimental timescale. However, partly equilibrated glasses represent glassy states of higher thermodynamic stability (Figure 1) showing lower enthalpy and denser packing. Instead of aging, glasses with similar thermodynamic stability can be obtained by sufficiently slow cooling. This implies glassy states produced whether by slow cooling or aging are thermodynamically indistinguishable. If glasses are re-heated across the glass transition the frozen-in structure becomes a liquid again.

It has recently been established that high stability glasses can be prepared by physical vapor deposition and some important properties of these materials have been investigated. Glasses produced by physical vapor deposition with substrate temperatures near $0.85 \quad T_{g}$ exhibit extraordinary properties including low enthalpy, low heat capacity, high kinetic stability, and high density [5-10]. While these enhanced features correspond qualitatively to properties expected for an aged glass, estimates indicate that an ordinary glass would need to be aged for one thousand to one million years in order to quantitatively attain the properties of these vapor-deposited systems. 
As vapor deposition produces thin films, a particularly useful approach for characterization is $\mathrm{AC}$ nanocalorimetry, a nanoscale calorimetry technique that can measure the heat capacity of films as thin as a few nanometers [11-13].

Here we characterize vapor-deposited glasses of toluene and ethylbenzene using an in situ differential AC nanocalorimetry device [13]. This technique is advantageous since it requires only small sample mass, enables the determination of $T_{g, d y n}$ in a broad frequency range, measures $T_{g, d y n}$ in the supercooled liquid state, and allows quasi-isothermal experiments. The high sensitivity of this device enables the measurement of very small differences in the heat capacities of vapordeposited and liquid-cooled glasses. Technical and experimental issues connected with the device are discussed in section 3 of this work. Toluene and ethylbenzene were chosen for vapordeposition because of the simplicity of their molecular structures. We study the properties of the as-deposited glasses as a function of substrate temperature and deposition rate (section 4). Temperature scanning experiments were performed to access the heat capacity and the onset temperature for the transition of the stable glass towards the supercooled liquid for as-deposited glasses, in comparison to glasses of the same substance prepared by cooling the liquid at a slow rate. The heat capacity provides information about the packing of the system and the onset temperature directly reports on the kinetic stability. The interrelationship between these two quantities over a wide range of deposition temperatures and rates will be examined for the first time. AC nanocalorimetry was also utilized for isothermal experiments, where the transformation of the as-deposited glass to the supercooled liquid was observed. The transformation time will be compared to the structural relaxation time to more precisely quantify the kinetic stability of the vapor-deposited glasses.

The aim of this work is to apply the highly sensitive in-situ AC nanocalorimetry to stable glasses of simple organic glass formers to learn about the general mechanism of stable glass formation. The link between the recently observed vapor-deposited stable glasses to the vapordeposited unstable glasses observed a few decades ago is still unknown and data in between has not yet been measured. Access to a broad deposition temperature range makes it possible to follow the evolution of the stability of vapor-deposited glasses from high stability to poor stability for the very first time. This work closes the gap and the data allows consistent explanations of the observations reported in the different works. 


\section{State of the art}

\subsection{Glasses and glass transition}

Figure 1 describes the freezing-in of glass forming materials at the glass transition. In the framework of thermodynamics this is the transition from a metastable (undercooled liquid) to a non-equilibrium (glass) state. A characteristic behavior of volume $V$, or Enthalpy $H$, or entropy $S$ is observed at the glass transition. If a constant cooling rate $\beta$ at constant pressure is used, as an example, the temperature coefficient of the enthalpy, the heat capacity $C_{p}$, undergoes a smooth step-like change at the glass transition. In Figure 1 it can be seen that in the non-equilibrium state (the glass) the slope of the enthalpy $H$ differs from the slope in the metastable state (SCL). Since the heat capacity $C_{p}$ at constant pressure is

$$
C_{p}=\left(\frac{\partial H}{\partial T}\right)_{p}
$$

a weaker temperature dependence yields to a smaller heat capacity and vice versa. Consequently, for the glass transition region, shown in Figure 1, where the enthalpy curve shows a smooth change in its temperature dependence the heat capacity exhibits a step. Other temperature coefficients like expansivity $\alpha$ and compressibility $\kappa$ show similar behavior.

The glass transition is a kinetic event caused by the dramatic slowing down of the molecular motions in the vicinity of the glass transition at decreasing temperature. A quantity that gives direct access to the molecular mobility of a liquid is the viscosity. The viscosity of a liquid is decreasing during cooling $[14,15]$. Viscosity changes many orders of magnitude when cooling from the melt to the glass transition. By means of the bulk viscosity the glass transition temperature $T_{g}$ is defined as the temperature at which the viscosity obtains a value of $10^{12} \mathrm{~Pa} \mathrm{~s}$ [16]. This value corresponds to a temperature where the structural relaxation time $\tau_{\alpha}$, which is the time needed for molecules to recover equilibrium, exceeds $100 \mathrm{~s}$. It is reasonable that the temperature at which the SCL falls out of equilibrium is dependent on the cooling rate $[17,18]$ since e.g. slower cooling increases the time available for molecular rearrangements and thus enables molecular motion to retain equilibrium down to lower temperature. An experimental cooling rate of about $10 \mathrm{~K} \mathrm{~min}^{-1}$ yields approximately the same value of $T_{g}$ as defined using the viscosity. The cooling rate dependence of the glass transition temperature is shown for the enthalpy and heat capacity of a common glass former in Figure 1. The green line in the enthalpytemperature plot represents the line for the liquid. By cooling with rate $\beta$ the system leaves the equilibrium line at $T_{g}$. A slower cooling rate $\beta$ ' causes the system to leave the equilibrium at a 
temperature $T_{g}{ }^{\prime}<T_{g}$ since longer time is available for molecular rearrangement. As a rule of thumb, changing the cooling rate by one order of magnitude will change the glass transition temperature by $3 \mathrm{~K}$ for fragile glass formers and $5 \mathrm{~K}$ for strong glass formers.
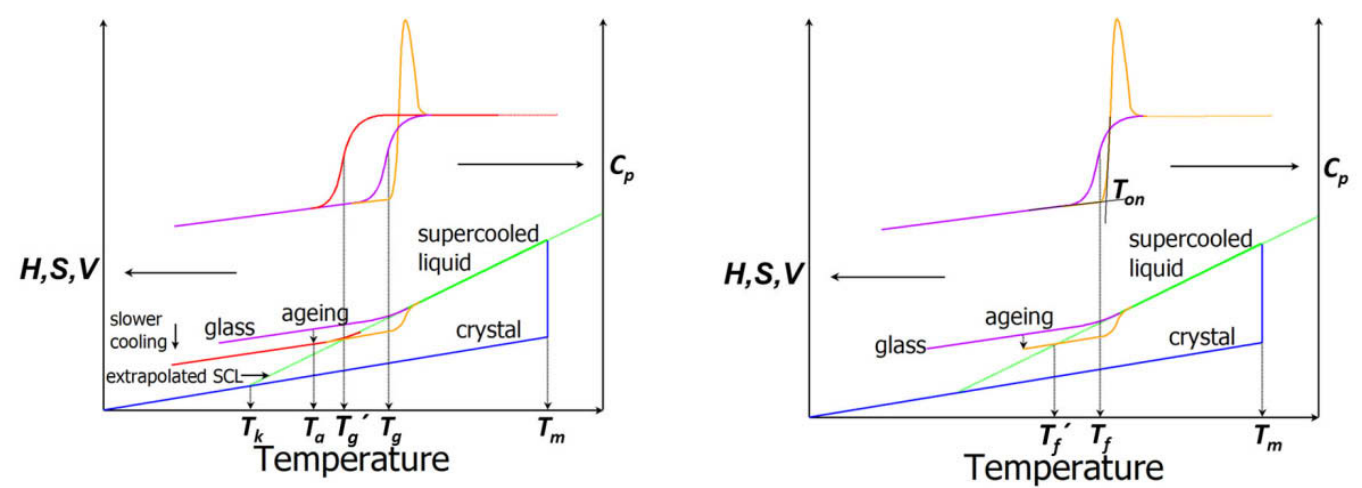

Figure 1: Schematic enthalpy over temperature plot for a glass forming material. Left: The blue line describes the equilibrium path when the material crystallizes with the sharp $1^{\text {st }}$ order phase transition at the melting temperature $T_{m}$. If crystallization can be avoided during cooling the liquid follows the supercooled liquid line (green) until it falls out of equilibrium at $T_{g}$, which depends on the cooling rate. Slower cooling yields a lower glass transition temperature, e.g. $T_{g}{ }^{\prime} . T_{k}$ is the Kauzmann temperature at which the curves for the supercooled liquid and the crystal hypothetically cross at very slow cooling. The non-equilibrium states of a slow and a fast cooled glass are shown as red and purple curves, respectively. The yellow curve represents the heating of the aged or slowly cooled glass at a heating rate which equals the cooling rate of the fast cooling. The upper panel of each plot shows the corresponding heat capacity curves. Right: The onset temperature $T_{\text {on }}$ is defined as the intersection of the tangents of the glassy heat capacity and the leading edge of the glass transition step. The fictive temperature $T_{f}^{\prime}$ of an aged glass is a measure of the thermodynamic stability and is defined as the intersection temperature of the enthalpy of the glass with the enthalpy of the extrapolated SCL. $T_{f}$ equals $T_{g}$ if aging can be avoided.

The difference in volume between the glasses obtained from cooling rates $\beta$ and $\beta$, originates mainly from a decreased free volume. Thus slower cooling yields glasses of smaller volume, lower enthalpy and lower entropy. Consequently the enthalpy $H$ (as a measure of the total energy of the thermodynamic system) of the glass obtained from the slower cooling rate $\beta$ ' attains a lower value than the glass obtained from faster cooling $\beta$ if compared at a temperature below $T_{g}{ }^{\prime}$ which means it is thermodynamically more stable (since it is closer to the equilibrium line). The progress towards lower enthalpy glasses is denoted as physical aging [19, 20]. Besides slow 
cooling, isothermal aging below $T_{g}$ (i.e. at the aging temperature $T_{a}$, see Figure 1) provides another way of yielding glasses with lower enthalpy. Glasses produced either by slow cooling or isothermal aging cannot be distinguished in the measured material properties of the subsequent heating curves.

Physical aging starts if relaxation towards the equilibrium state begins, i.e. in the nonequilibrium state. This means glasses commonly partly equilibrate below $T_{g}$ during cooling and re-heating. Thus the thermodynamic stability of the re-heated glass near $T_{g}$ is higher than for the freshly cooled glass and the thermodynamic state of the re-heated glass corresponds to a glass obtained by slower cooling than really used for freezing-in.

During re-heating of an annealed glass the frozen-in and relaxed cooperative motions of the glass "melt" at higher temperature as compared to the freezing-in process which leads to "overheating" of the glass and results in a steep increase in the enthalpy and consequently an enthalpy relaxation peak in the heat capacity (see Figure 1) that has its origin in an enhanced relaxation speed due to the delayed onset of the glass transition [18]. This is a consequence of the higher kinetic stability of the slowly cooled glass and is quantified by the higher onset temperature $T_{o n}$. Due to the higher $T_{o n}$ of the re-heated glass its enthalpy crosses the extrapolated equilibrium line on the way to heating to $T_{o n}$. The temperature of intersection is denoted as the fictive temperature $T_{f}$ which is a measure of the thermodynamic stability of the glass [20,21].

After the frozen-in structure is "molten" $H$ again follows the equilibrium line of the SCL (if no crystallization occurs) and molecular mobility increases during further heating. At some point molecular rearrangement becomes fast enough to follow applied periodic perturbations like in $\mathrm{AC}$ calorimetry or dielectric spectroscopy. The latter probes, using an applied oscillating electric field, fluctuations of the molecular dipoles and thus relaxational dynamics. In dielectric experiments, at constant sample temperature above $T_{g}$, the frequency $\omega$ of the applied electric field is varied. The frequencies used span a wide range from e.g. $10^{-4} \mathrm{~Hz}$ up to $10^{12} \mathrm{~Hz}$. When the frequency is too high, the dipoles are too slow to contribute to the response. Lower frequencies enable contributions of the fluctuations to the response and an increase in the real part of the measured complex dielectric permittivity is observed (Figure 2). This is accompanied by a peak in the imaginary part of the permittivity. The peak-frequency together with the sample temperature denotes a tuple defining the so-called dynamic glass transition temperature $T_{g, d y n}$. This means $T_{g, d y n}$ is a function of the frequency of the perturbation. For each temperature $T>T_{g}$ a frequency $\omega$ exists where a liquid exhibits a peak in the imaginary part of the permittivity $\left(T_{g, d y n}\right)$ and vice versa. 


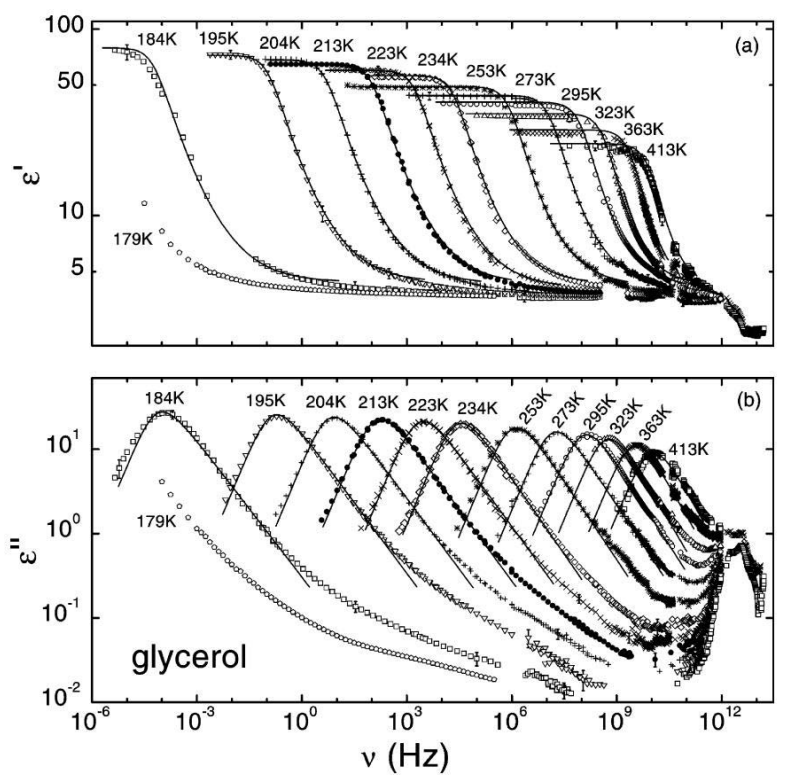

Figure 2: Frequency dependence of the real and the imaginary parts of the dielectric permittivity in glycerol $\left(T_{g}=185 \mathrm{~K}\right)$ at various temperatures. The sub- $T_{g}$ curve at $179 \mathrm{~K}$ was measured in thermal equilibrium after five weeks aging time. The lines are fits. Pictures taken from [22].

If the response of the molecular rearrangement to a small periodic temperature perturbation is measured as a function of oven temperature one observes a step-like change in the real part of the response similar to the change in heat capacity for the freezing-in process. The response to periodic temperature oscillations is denoted as the reversing heat capacity or modulus of complex heat capacity. The step in the reversing heat capacity is the dynamic glass transition (see Figure 3). The dynamic glass transition is commonly independent on the thermal history of the material since complete relaxation in the vicinity of $T_{g, d y n}$ occurs on short timescales. Again $T_{g, d y n}$ depends on the used frequency of thermal oscillation.

It is important to distinguish between the glass transition temperature $T_{g}$ and the dynamic glass transition temperature $T_{g, d y n}$ since the former describes an equilibrium-to-non-equilibrium transition, while the latter occurs in the thermodynamic equilibrium. The molecules of a probed sample stay immobile on the timescale of the oscillation for temperatures in the vicinity of the glass transition temperature $T_{g}$ as determined by adiabatic calorimetry or DSC. Only in the metastable thermodynamic equilibrium above $T_{g}$ the molecular rearrangement can follow the oscillation which is measured by a step in the real part of the susceptibility $c^{\prime}$ and a peak in the imaginary part $c^{\prime \prime}$ (see Figure 3). The peak denotes the temperature $T_{g, d y n}$ of the dynamic glass transition. Depending on the frequency used for the dynamic experiment the dynamic glass transition temperature $T_{g, d y n}$ is shifted to higher temperatures compared to $T_{g}$. All dynamic 
methods like dielectric spectroscopy, AC calorimetry and the $3 \omega$-method reveal the dynamic glass transition in the supercooled liquid.

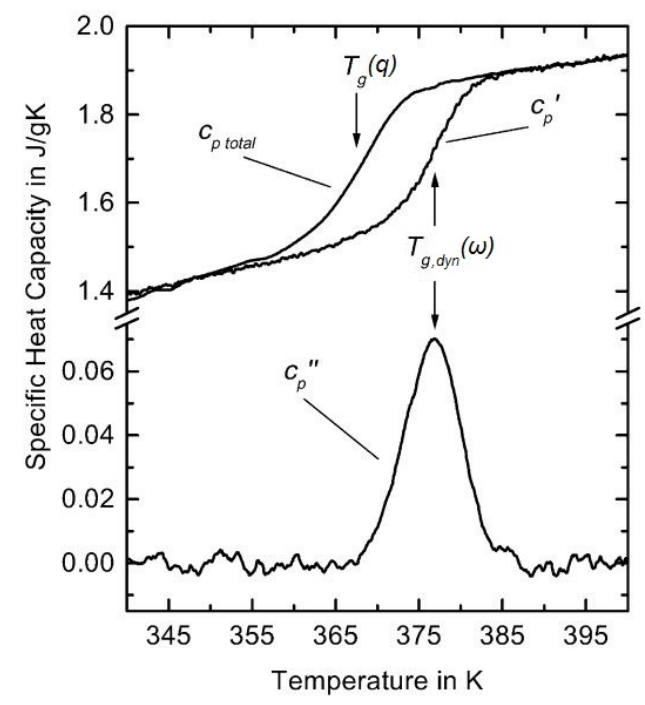

Figure 3: Total specific heat capacity $\left(c_{p, \text { total }}\right)$, real $\left(c_{p}\right)$ and imaginary part $\left(c_{p}{ }^{\prime \prime}\right)$ of the complex specific heat capacity of polystyrene determined from temperature modulated DSC (TMDSC). Picture taken from [23] and modified.

Re-heating a highly relaxed glass yields a high $T_{\text {on }}$ of the "structure melting" which can be above $T_{g}$ of the non-relaxed glass i.e. determined from the freezing-in. If very small frequencies of the temperature oscillation for the measurement of the complex specific heat capacity are used $T_{g, d y n}$ gets close to $T_{g}$. However, response from the temperature oscillation can be obtained only above $T_{o n}$. Thus the complex heat capacity is affected by the higher onset [24] (Figure 4). The characteristic enhanced relaxation towards equilibrium as seen in the total heat capacity during reheating is observed in the modulus of the complex heat capacity as well. The phase peak is similarly shifted with increasing $T_{o n}$. 


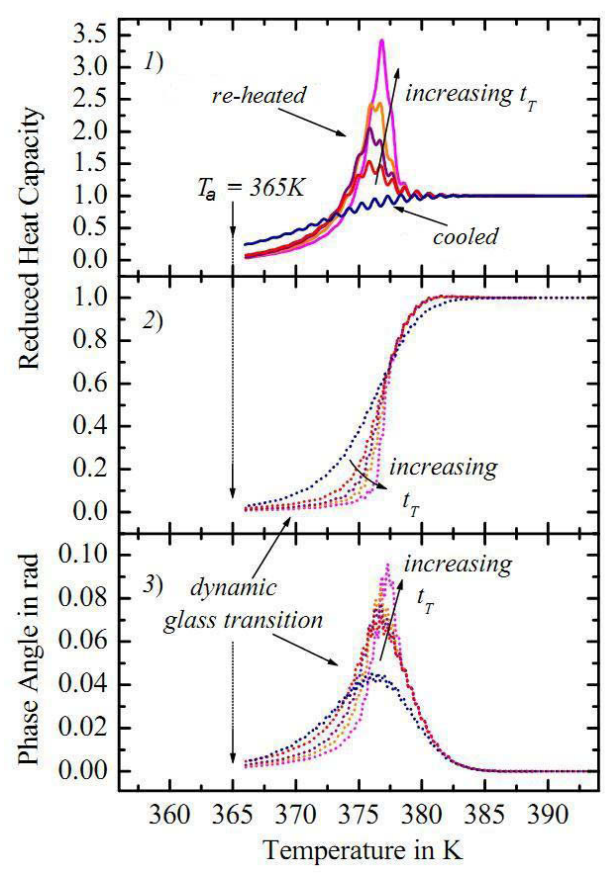

Figure 4: Influence of aging on complex heat capacity. 1) total heat capacity of annealed and re-heated glasses. 2) modulus of the complex heat capacity. 3) phase of the complex heat capacity. The glasses were annealed at $T_{a}$ for $0 \mathrm{~h}, 1 \mathrm{~h}, 2 \mathrm{~h}, 4 \mathrm{~h}$. Picture taken from [24].

Aged glasses always influence the shape of the glass transition step and can exhibit a higher glass transition temperature. Depending on the kinetic stability and the used frequency this can also be true for the dynamic glass transition step. However, e.g. AC calorimetry can shift $T_{g, d y n}$ to exceed $T_{o n}$ by making use of higher frequencies. Through this $T_{g, d y n}$ can be adjusted to avoid any influence of the higher $T_{o n}$ or to have both temperatures close to another, as is the case in this work.

The structural relaxation time $\tau_{\alpha}$ becomes smaller and smaller with increasing temperature and allows for measuring the response to the applied oscillating perturbation for even higher frequencies $\omega_{\alpha}=1 / \tau_{\alpha}$. Thus the loss peak shifts to higher frequencies for higher temperatures. This gives a functional relation between the structural relaxation time and the dynamic glass transition temperature. This functional relation is material specific and can be distinguished regarding the shape of the function. The function of the logarithm of the relaxation time versus reciprocal temperature is well curved for fragile glasses whereas for strong glasses the function is moderately curved (Figure 5) [25]. In other words: strong glasses show Arrhenius-like behavior 


$$
\tau_{\alpha}(T)=\tau_{0} \exp \left(\frac{E}{k_{B} T}\right)
$$

and fragile glasses Vogel-Fulcher-Tammann (VFT-) behavior [14, 15, 26]

$$
\tau_{\alpha}(T)=\tau_{0} \exp \left(\frac{D T_{0}}{T-T_{0}}\right)
$$

$E$ is the apparent activation energy, $k_{B}$ is the Boltzmann constant, $D$ and $T_{0}$ are material dependent parameters. $T_{0}$ is often denoted as the Vogel-temperature, which is the temperature where the structural relaxation time gets infinitely long. However, the physical meaning of $T_{0}$ is still unclear. Often $D$ and $T_{0}$ are lumped together into a parameter $B$.

Glass formers are distinguished with regards to their steepness index or fragility $m$. The fragility $m$ is defined as the derivative of the logarithm of the structural relaxation time over $T_{g} / T$ as the temperature approaches $T_{g}$

$$
m=\left.\frac{d \log \left(\tau_{\alpha}\right)}{d\left(T_{g} / T\right)}\right|_{T=T_{g}}
$$

and varies from a few tens (strong glasses, e.g. $m$ (betaine phosphate(60):betaine phosphite $(40))=14.2$ [27]) to above 200 (fragile glasses, e.g. $m$ (polyetherimide) $=214$ [28]) Figure 5 shows the shape of the VFTs for glasses of different fragilities in a so-called Angell plot.

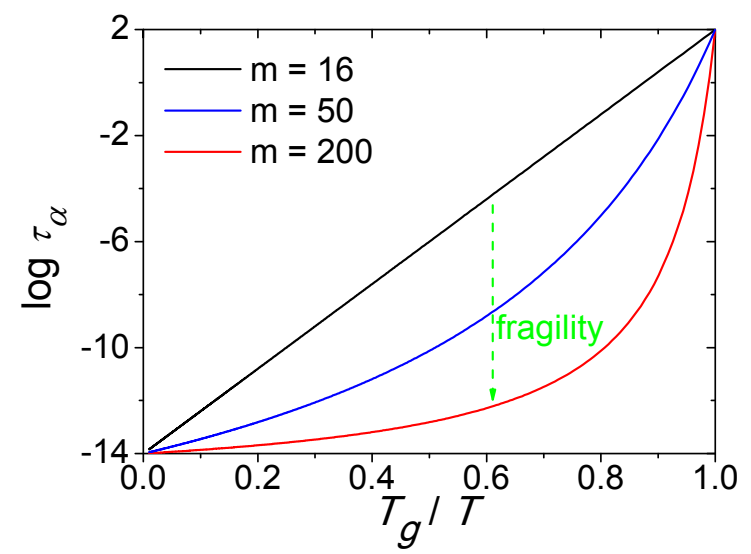

Figure 5: Angell plot of the VFTs of glass forming materials for three different fragilities that can be determined from measurements of the frequency dependent structural relaxation time $\tau_{\alpha}$ by (4). An almost straight line represents a strong glass former showing approximately Arrhenius-behavior and a curved line a fragile glass former showing VFT-behavior. 
Calorimetry in general can measure the heat capacity or the enthalpy of a material and this has provided many important insights about glasses and supercooled liquids over the course of the last century. Low temperature anomalies in the heat capacity of glasses have provided important insights into fundamental distinctions between amorphous and crystalline systems [29]. Calorimetry is commonly used to measure the increase in the heat capacity that occurs at the glass transition temperature $T_{g}$; it has been suggested the heat capacity increase $\left(\Delta C_{p}\right)$ is correlated with the kinetic fragility of the glass former, at least for a subset of materials [30, 31]. Calorimetry also provides a means of investigating the Kauzmann entropy crisis and its potential resolutions [3234]. More generally, calorimetry provides critical information about the potential energy landscape, which controls the thermodynamics and dynamics of supercooled liquids and glasses $[16,35-37]$.

In this work $T_{g, d y n}$ is solely measured by means of AC nanocalorimetry. With this technique temperature oscillations at frequencies that are commonly orders of magnitude higher than the frequency of $10^{-2} \mathrm{~Hz}$ typically used in temperature-modulated differential scanning calorimetry (TMDSC) are applied [38]. Depending on the frequency used for the AC calorimetric experiment, the dynamic glass transition $T_{g, d y n}$ is shifted compared to $T_{g}$ as determined by DSC at $10 \mathrm{~K} / \mathrm{min}$. As a rule of thumb, $T_{g}$ equals $T_{g, d y n}$ at about $10^{-3} \mathrm{~Hz}$ (or a structural relaxation time $\tau_{\alpha}$ of about $100 \mathrm{~s})$.

\subsection{Vapor deposited glasses}

In addition to slow cooling from the melt, glasses can be produced by vapor deposition [39]. In a typical physical vapor deposition (PVD) process deposition onto the substrate can be realized by different techniques e.g. sputter deposition, electron beam deposition or evaporative deposition.

\subsubsection{Two different observations of the stability of vapor-deposited glasses}

Early works reported vapor-deposited glasses exhibit higher enthalpy than common liquid-cooled glasses and thus are located higher in the energy landscape. High enthalpy glasses were first successfully produced via PVD in the group of Suga [39]. Even though PVD and chemical vapor deposition (CVD) were used before to produce samples for calorimetric measurements no glass transition was observed. Sugisaki et al. used an adiabatic calorimeter that needed a large amount of deposited material ( $1 \mathrm{~g}-2 \mathrm{~g}$ of methanol) which takes about 10 to 20 
hours of deposition at high deposition rates. The temperature of the sample cell was kept between $94 \mathrm{~K}$ and $97 \mathrm{~K}$ which is about 0.91 to 0.94 of the glass transition temperature $T_{g}$ of methanol $(103 \mathrm{~K})$. Two samples were deposited at different deposition rates, sample 1 at $0.101 \mathrm{~g}$ /hour and sample 2 at $0.366 \mathrm{~g} /$ hour. Sugisaki et al. found that only sample 1 is fully amorphous whereas sample 2 contains only $37.4 \%$ amorphous methanol. This is explained by the heat of condensation raising the temperature of the sample cell to above the crystallization temperature. In a later work by Haida et al. [40] during the production of vapor deposited samples of chloroform and propene the sample cell was kept at liquid hydrogen temperature. A more detailed discussion of low temperature vapor deposited glasses of butyronitrile $\left(T_{g}=97 \mathrm{~K}\right)$ measured with an adiabatic calorimeter can be found in [41]. Here the depositions were performed at two different temperatures 0.69 and 0.41 of the glass transition temperature. The deposition rates were kept at about $10 \mathrm{mg} /$ hour. The authors found the same glass transition temperatures for both samples. An important result, however, is that they found the configurational enthalpy of the sample deposited at $0.41 T_{g}$ reached a value of more than $1.3 \mathrm{~kJ} \mathrm{~mol}^{-1}$ for the lowest temperature which is more than $40 \%$ of the enthalpy of crystallization at $110 \mathrm{~K}$ (see Figure 6). For a normal glass cooled from the liquid they found a configurational enthalpy of less than $0.1 \mathrm{~kJ} \mathrm{~mol}^{-1}$. The configurational enthalpy of the sample deposited at $0.69 T_{g}$ lies in between. That indicates the lower the substrate temperature during deposition the higher the configurational enthalpy. The authors also measured spontaneous heat evolutions at low temperatures well below the glass transition temperature. These spontaneous heat evolutions correspond with the change in configurational enthalpy. This mechanism was explained as a low temperature relaxation process which must be different from that of liquid-cooled samples. Since this relaxation took place at a state of very high configurational enthalpy it seems that the molecules deposited on a cold substrate are arrested in their arrangement specified by the deposition temperature and the molecules have weaker molecular interaction among themselves than in the liquid-cooled sample.

As an explanation the authors proposed to understand the spontaneous heat evolution as a formation of cluster structures during deposition. The large cluster rearrangement needs large energy of activation and thus the size of the clusters formed during deposition must strongly depend on the deposition temperature. The size of the clusters represents the range of order. If the temperature is raised, the activation of a larger unit of molecular arrangement results in a further development of short-range order. However, this indicates that liquid-cooled and vapor-deposited samples would have a similar structure at the glass transition region consisting of clusters of a size characterized by the temperature. The authors stated: "the low temperature enthalpy relaxation of the vapor-deposited sample is well interpreted as due to a less-developed cluster structure, 
and the structure of vapor-deposited and liquid-cooled samples should be clearly distinguished by the average sizes of the clusters" [41].

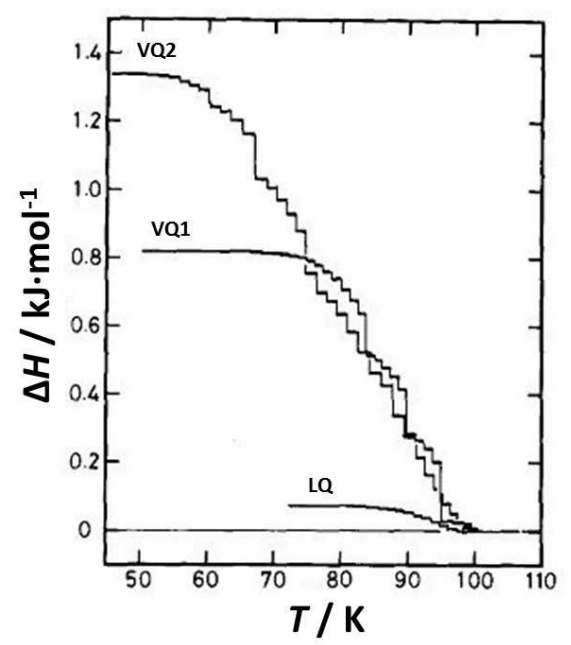

Figure 6: The configurational enthalpy of vapor-deposited (vapor-quenched VQ) and liquid quenched (LQ) samples of butyronitrile. The vapor-deposited samples with a mass of about $1 \mathrm{~g}$ were made with a deposition rate of about $10 \mathrm{mg} \mathrm{h}^{-1}$. The substrate temperature during deposition was held between $66 \mathrm{~K}-68 \mathrm{~K}$ (VQ 1) and $40 \mathrm{~K}$ (VQ 2). The $T_{g}$ of butyronitrile is about $97 \mathrm{~K}$. Thus the samples were deposited at $0.69 T_{g}$ and $0.41 T_{g}$ respectively. Picture taken from [41].

A later study of 1-pentane [42] yielded comparable qualitative results. Here the substrate temperature during deposition was held at temperatures between $38 \mathrm{~K}$ and $47 \mathrm{~K}$ which is about $0.54 T_{g}$ and $0.67 T_{g}$. To produce the needed sample mass of about $1 \mathrm{~g}$ took 50 hours. The film thickness inside the calorimetric cell was estimated to be $100 \mu \mathrm{m}$ which corresponds to a deposition rate of about $0.6 \mathrm{~nm} \mathrm{~s}^{-1}$.

Since in the adiabatic experiments in the group of Suga the sample mass had to be sufficiently high (about $1 \mathrm{~g}$ ) the problem of heat evolution during the deposition process forced the experimenters to stick to sufficiently low deposition temperatures and thus the common view of low temperature vapor-deposited glasses at this time was somewhat transferred to vapordeposited glasses in general.

Recently PVD was found to produce glasses with unusual stability. Since the needed sample mass used for the recent measurements was relatively small and thus heat input due to heat of condensation was low and compensable the problem of heat evolution during the deposition process did not arise. This allowed deposition temperatures close to $T_{g}$ which yields stable glasses. A first indication of the unusual stability of some vapor-deposited glasses was the observation of 
Mapes et al. that the effective diffusion coefficient of vapor-deposited o-terphenyl depended upon the deposition temperature [43]. Later high stability of vapor-deposited glasses of tris(naphthylbenzene) was directly observed in neutron reflectivity measurements by Swallen et $a l$. [8]. Afterwards other substances were shown to form stable glasses by vapor-deposition using different techniques, e.g. indomethacin (DSC [5], WAXS [44], AC-nanocalorimetry [45] and ellipsometry [10]), toluene and ethylbenzene (fast scanning [46] and adiabatic calorimetry [7], light interference [6]), propylbenzene and isopropylbenzene (light interference [47]), and nifedipine, felodipine and phenobarbital (DSC [48]).

Stable glasses exhibit a low fictive temperature $T_{f}$ (high thermodynamic stability) as determined from DSC measurements and a high onset temperature $T_{\text {on }}$ of the glass transition which can be attributed to a higher kinetic stability of the glass. The molecules are stuck in their immobile as-deposited glassy state until a certain temperature (above $T_{g}$ ) is reached allowing the molecules to become "unjammed" and relax towards their SCL state. For thin films it is assumed this takes place via a surface-initiated growth front that propagates with constant velocity [49].

Since these first observations, various techniques yielded various properties of vapordeposited glasses to be extraordinary. For deposition temperatures in the vicinity of $0.85 T_{g}$ vapordeposited glasses exhibit low enthalpy [5, 7, 34, 46, 50], the heat capacity of the glass drops by about 4\% [50-53], high density (1.3\% denser for TNB) [10], high mechanical moduli (up to 15\% increased) $[54,55]$ and they can resist water uptake [44]. 2D WAXS measurements by Dawson et al. revealed stable glasses are anisotropic [56].

\subsubsection{Possible mechanism of stable glass formation}

What is the mechanism leading to stable glass formation? One explanation is based on an enhanced molecular mobility at the surface layer or more precisely at the sample-vacuuminterface. Evidences of an existing mobile surface layer of glassy polymer films can be found in numerous publications [57-61]. As estimated by Swallen et al. [8] at $0.85 T_{g}$ the surface layer for TNB exhibits a surface diffusion coefficient as commonly found near $T_{g}$ in the bulk.

During a slow deposition process at about $0.85 T_{g}$ it is assumed the surface molecules show such a sufficiently high mobility that they are able to find a lower energy configuration before they get buried by the next layer of molecules and join the bulk. The time for configurational sampling is hereby in the order of several structural relaxation times $\tau_{\alpha}$. A higher deposition rate limits the time for configurational sampling which yields the glass to be less stable compared to a slowly deposited glass. The stability of such glasses is also influenced by the 
substrate temperature. Lowering the substrate temperature lowers the mobility of the surface molecules which again limits the time for configurational sampling. If the time for configurational sampling is still long enough the molecules are able to equilibrate. Below a certain deposition temperature, that is about $0.85 T_{g}$ for IMC and TNB, the mobility of the surface layer becomes so slow that further equilibration of the molecules is not possible. Lower substrate temperatures thus do not allow the surface molecules to equilibrate before they join the bulk and the vapor-deposited glass becomes less stable as compared to glasses produced at $0.85 T_{g}$.

Some investigations of the sub- $T_{g}$ surface dynamics of polymers gave evidence of a mobile surface layer penetrating several $\mathrm{nm}$ deep into the sample [57, 62-65]. Recently Paeng et $a l$. developed an optical method for studying molecular mobility of thin polymer films by dyereorientation [66]. For supported thin films of polystyrene, poly(methyl methacrylate) and poly(2-vinyl pyridine) [67] the mobile layer thickness decreased slowly with temperature and was still nonzero at $0.9 T_{g}$. This temperature dependent mobile layer thickness was consistent with what Swallen [8] found for TNB.

\subsubsection{Influence of substrate temperature}

Kearns et al. did several depositions of IMC at different substrate temperatures below the glass transition temperature (where $\tau_{\alpha}=100 \mathrm{~s}$ ) into a DSC aluminum pan and determined the fictive temperature [5] via DSC measurements (see Figure 7). They found the fictive temperature, as a parameter characterizing the thermodynamic stability of the vapor deposited glasses, to decrease with decreasing substrate temperature starting from almost $T_{g}$ until a minimum at $0.85 T_{g}$ is reached showing a $T_{f}$ as much as $40 \mathrm{~K}$ lower compared to glasses prepared by cooling a liquid. Further decrease of the substrate temperature yields an increasing $T_{f}$ since the structural relaxation time of the mobile surface layer became too long to let the molecules arrange into better configurations in the mobile layer before they get buried by the next molecules coming from the gas phase and forming the new surface of the sample.

The kinetic stability of an as-deposited sample is characterized by the onset temperature $T_{\text {on }}$ of the enthalpy relaxation peak and was determined for IMC from DSC curves (see Figure 7). The enthalpy relaxation peak shifts to higher temperatures if approaching a substrate temperature of $0.85 T_{g}$ from above and starts to drop on the temperature axis if going to even lower substrate temperatures. Following this behavior $T_{o n}$ is increasing and decreasing as well. $T_{o n}$ is interpreted as follows: As long as the sample remains in the as-deposited stable state molecular packing is efficient and thus thermal energy can only contribute to small vibrational motions of the molecules and heat capacity remains low. Only when the stable glass transforms to the 
supercooled liquid above $T_{\text {on }}$ additional modes are excited. At $T_{o n}$, the molecules start to move cooperatively, and an increase in heat capacity is observed as a consequence of configurational reorganization.
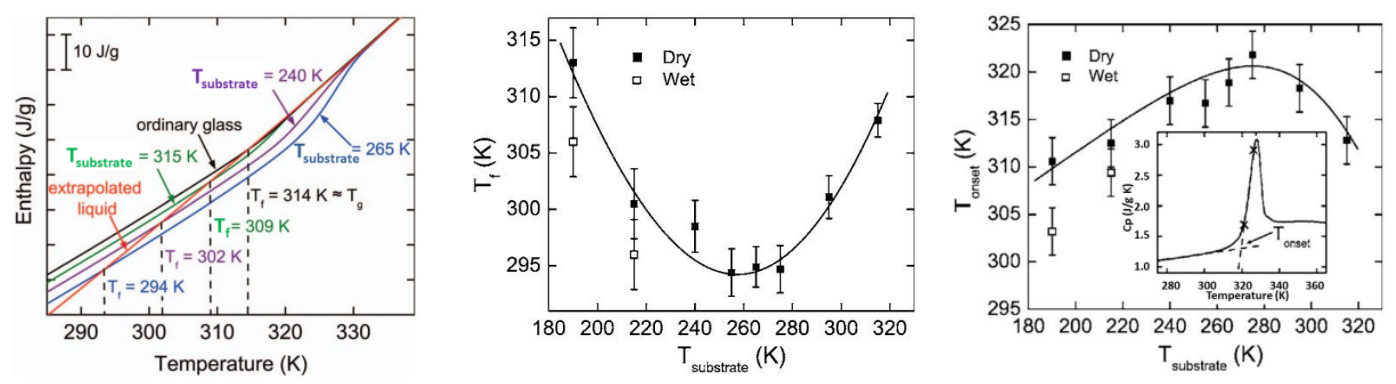

Figure 7: Left: Enthalpy curves of IMC for the extrapolated liquid, the ordinary glass and the as-deposited glasses for different substrate temperatures $T_{\text {substrate }}$. The deposition rate was held constant for all depositions at about $(15 \pm 3) \mathrm{nm} \mathrm{s}^{-1}$. The shown fictive temperatures $T_{f}$ for IMC are determined from the intersection of the glassy enthalpy with the extrapolated liquid enthalpy. Middle: The determined $T_{f}$, plotted as a function of $T_{\text {substrate, }}$ for dry and wet samples. The solid line is a guide to the eye. Right: Kinetic stability as indicated by the onset temperatures $T_{\text {on }}$ for samples of IMC deposited at various $T_{\text {substrate. }}$ The solid line is a guide to the eye. The inset shows the definition of the onset temperature $T_{\text {on }}$ from the heat capacity curve as indicated by the intersection of the dashed lines. Pictures taken from [5].

Compared to commonly aged glasses the enthalpy relaxation peak of as-deposited glasses appears more pronounced [34]. Due to the low fictive temperature and the late onset of the glass transition, subsequent molecular rearrangement is enhanced and gives a sharp and high enthalpy overshoot which is shifted to higher temperatures compared to liquid-cooled glasses.

\subsubsection{Influence of deposition rate}

Substrate temperature is not the only factor controlling the stability of as-deposited glasses. The formation of stable glasses is always an interplay between substrate temperature and deposition rate. The deposition rate limits the time surface molecules have for configurational sampling before they are buried and join the bulk. Thus low deposition rates give the molecules more time for configurational sampling.

Kearns et al. investigated the deposition rate dependence on the enthalpic stability of glasses of IMC and TNB by DSC measurements [34]. Holding the substrate temperature at about 
$0.84 T_{g}$ the deposition rate was changed by 2 orders of magnitude. (see Figure 8 ) They found a decrease of about $10 \mathrm{~K}$ in the fictive temperature for vapor-deposited glasses of IMC and TNB. Accordingly kinetic stability increased with lower deposition rate.
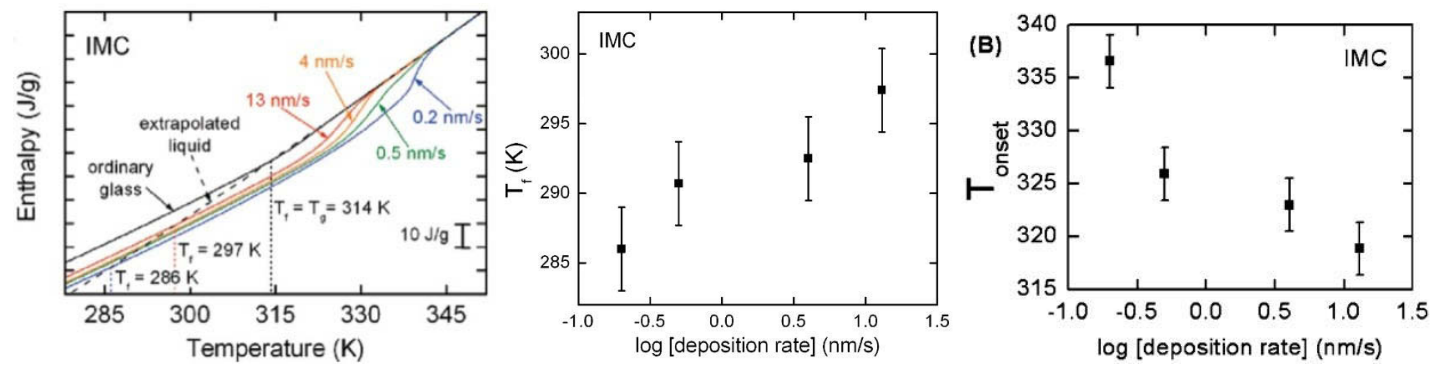

Figure 8: Left: Enthalpy curves of IMC for the extrapolated liquid, the ordinary glass and the as-deposited glasses for different deposition rates. The substrate temperature $T_{\text {substrate }}$ was held constant for all deposited samples at about $0.84 T_{g}$. The shown fictive temperatures $T_{f}$ for IMC are determined from the intersection of the enthalpy with the extrapolated liquid enthalpy. Middle: The determined $T_{f}$, plotted as a function of deposition rate. Right: Kinetic stability as indicated by the onset temperatures $T_{\text {on }}$ for samples of IMC deposited at various deposition rates. Pictures taken from [34].

In recent work by León-Gutierrez et al. no indication of a deposition rate dependent stability of vapor-deposited glasses of toluene and ethylbenzene was found by fast scanning calorimetry [46]. In their work the experimenters were limited to deposition rates between 0.001 $\mathrm{nm} \mathrm{s}^{-1}$ and $0.1 \mathrm{~nm} \mathrm{~s}^{-1}$.

\subsubsection{Transformation of a stable glass to the super cooled liquid}

The transformation of a stable glass to the supercooled liquid (SCL) can be followed by different methods. Swallen et al. $[49,68]$ observed the isothermal transformation of layered films of TNB and IMC using secondary ion mass spectroscopy (SIMS) above $T_{g}$. (see Figure 9 left) They have shown that the isothermal transformation of stable glasses to the SCL proceeds via a surface-initiated growth front that propagates linearly in time. Also a growth front progressing from the glass/substrate interface was observed in some cases. Swallen et al. proposed [49] that growth fronts arise as a result of kinetic facilitation [69-71] and the particular initial conditions of the experiments. Kinetic facilitation expresses the idea that mobility is locally required to create mobility in an otherwise jammed system. For thin films, surfaces and interfaces can initiate the transformation with little or no induction period. For the free surface Swallen et al. supposed that 
this occurs because of the high mobility of the surface layer. This gives rise to a planar growth front.

AC calorimetry offers another possibility to follow the transformation of the stable glass to the SCL via reversing heat capacity measurement if the onset temperature of the transformation is close to or above the dynamic glass transition temperature for the used frequency of temperature oscillation. The high sensitivity of differential AC chip-nanocalorimetry allows the detection of very small differences in glassy heat capacity. Since small samples are needed the deposition process is considerably shortened compared to adiabatic calorimetry. This allows high throughput. Kearns et al. first did ex-situ differential AC-calorimetric experiments on vapordeposited IMC and varied the film thickness (Figure 9 right). The results were consistent with the picture of a linearly propagating growth front transforming the stable glass into the SCL.
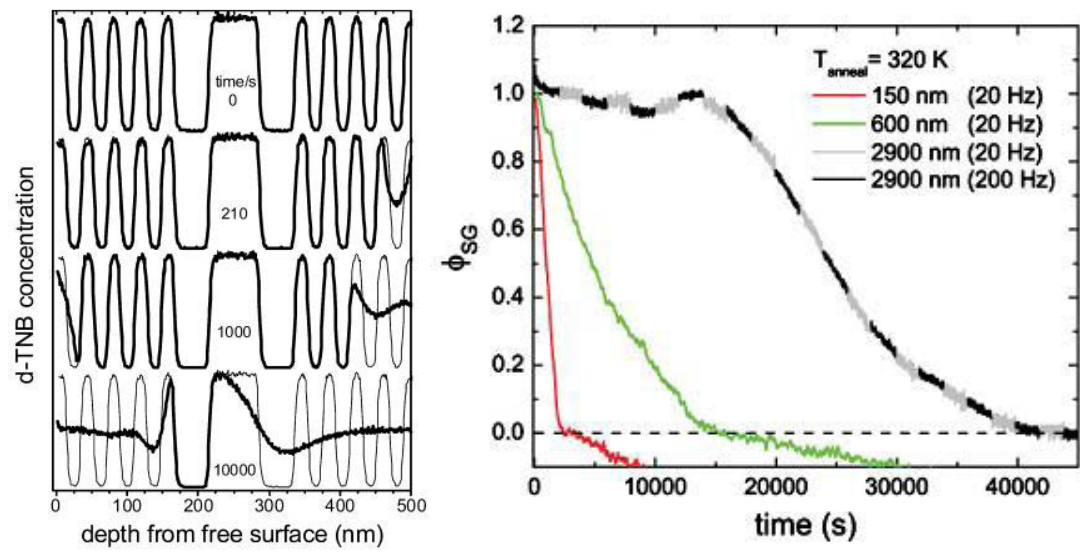

Figure 9: Left: Inhomogeneous evolution of $d-T N B / h-T N B$ concentration profiles for a stable glass during annealing at $352 \mathrm{~K}$ for the times indicated. These samples were deposited at $295 \mathrm{~K}$ at $0.2 \mathrm{~nm} \mathrm{~s}^{-1}$. Growth fronts are progressing from the free surface and the glass/substrate interface. Right: Isothermal transformation of stable glasses of IMC for different film thicknesses. The fraction of the sample that is a stable glass $\Phi_{S G}$ as a function of time. For all three experiments the deposition parameters were equal. Pictures taken from [68] (left) and [51] (right).

To gain an even higher sensitivity and bypass experimental difficulties we use in-situ AC chip-nanocalorimetry for stable glass investigation. The materials of choice are toluene and ethylbenzene since they are of simple molecular structure and easy to handle. The following chapter describes the used experimental setup enabling in-situ measurements of vapor-deposited thin films of low $T_{g}$ compounds by differential AC chip-nanocalorimetry. 


\section{Experimental}

\subsection{Chamber setup}

\subsubsection{Requirements for in-situ AC calorimetry of vapor-deposited samples}

The aim of the present study is in-situ investigation of vapor-deposited stable glasses by differential AC chip-nanocalorimetry. Therefore deposition and calorimetric measurement have to be able to be performed inside an ultra-high vacuum (UHV) chamber equipped for PVD. To allow high throughput and re-use of the sensors highly volatile small molecular glass formers, which will vaporize at room temperature, are favored. To produce a vapor-deposited sample onto a substrate requires a pressure inside the deposition chamber lower than the vapor pressure of the material to be deposited and a substrate temperature that is held below the evaporation/sublimation temperature of the material to be deposited or, as for the purpose of this work, even below $T_{g}$. Regarding the materials under investigation here this requires a sensor holder, a copper block, containing the chip calorimeters, which can be cooled to cryogenic temperatures and heated. In the following chapters the chamber setup, the experimental technique, temperature calibration as well as some experimental issues are discussed.

\subsubsection{Vacuum chamber}

The vacuum system consists of a CF100 double-cross chamber evacuated by a turbo molecular pump from Pfeiffer Vacuum GmbH in series with a Trivac scroll pump from Oerlikon Leybold Vacuum GmbH reaching a base pressure of about $5 \cdot 10^{-7} \mathrm{~Pa}$ (Figure 10). The pressure is monitored by a two-stage full range gauge from Pfeiffer Vacuum GmbH. The gauge is switched off during deposition and measurements on very thin films to prevent damage of the films [72]. A fine leak valve from MDC Vacuum Products LLC is attached to the chamber for flooding with dry nitrogen or any other gas, if needed. The top flange was specially manufactured by Vacom GmbH including a Sub-D 25 electrical feedthrough to connect the sensors, a 10 pin electrical feedthrough for temperature control and deposition control as well as a CF100-40 reduction to attach cooling pipes and the quartz crystal microbalance (QCM).

In order to achieve deposition rates from $100 \mathrm{~nm} \mathrm{~s}^{-1}$ down to $0.01 \mathrm{~nm} \mathrm{~s}^{-1}$ a sample reservoir is connected to the chamber via an ultra-fine leak valve in series with a bi-stable electromagnetic angle valve, both from MDC Vacuum Products LLC (see Figure 11). The sample 
reservoir of $10 \mathrm{~cm}^{3}$ and a leading tube can be heated if necessary. The $4 \mathrm{~mm}$ inner diameter tube leads the sample material into the chamber for deposition on the sample sensor and the QCM. The end of the tube is about $5 \mathrm{~cm}$ away from the sensor and the QCM. The molecules of the material under investigation arrive at the sensor directly from the source tube and also indirectly after colliding with the warm solid surfaces in the chamber. Thus molecules with a variety of deposition angles contribute to the film. But this angle range is limited to approx. $60^{\circ}$ by recessing the sensor into a $9 \mathrm{~mm}$ deep hole with a diameter of $9 \mathrm{~mm}$. Molecules colliding with the inner walls of the hole stick to these walls since the walls are at a temperature where the sticking coefficient is about 1 and thus cannot contribute to the film.

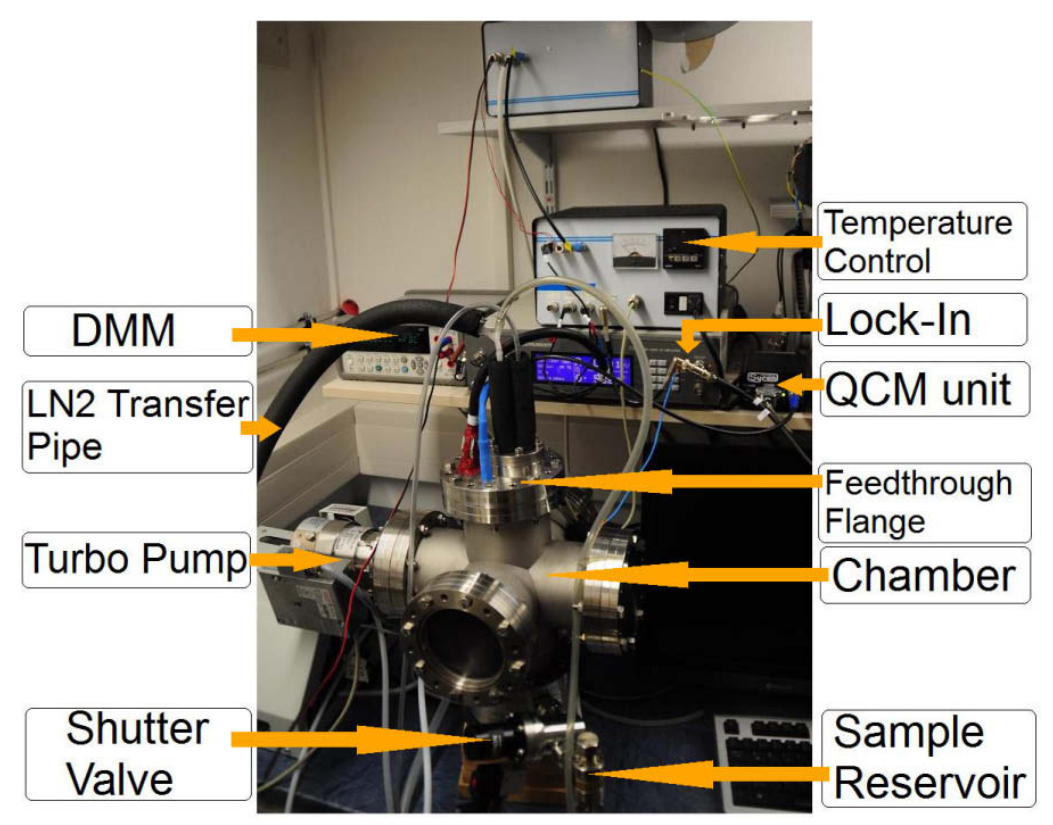

Figure 10: Photograph of the peripheral chamber setup.

A bi-stable electro-magnetic angle valve from MDC Vacuum Products LLC, located between the fine leak valve and the leading tube, allows the deposition to be immediately started or stopped without adjusting the leak valve. This electro-pneumatic shutter valve can be addressed via software (LabView) to close and open, automatically. This feature yields highly reproducible deposition rates. The deposition rate is monitored with a QCM utilizing an AT-cut quartz crystal located in the very center of the temperature controlled copper block next to the sample sensor. At the deposition temperature, the AT-cut crystal is far below the normal working temperature of such crystals, which is room temperature, so the measured deposition rate is only used to qualitatively monitor the deposition process. Moreover the angular distribution of molecules hitting and sticking to the QCM is much broader since the QCM is not located in a deep hole. This implies that the number of molecules per time and unit area sticking to the QCM is different (higher) than sticking to the sensor surface. The QCM was therefore only used to monitor the 
deposition process. Thus the thickness of the film deposited onto the sensor membrane cannot be determined from the QCM.

While the bi-stable electro-magnetic angle valve is closed some vapor of the sample material accumulates in the space between the shutter valve and the fine leak valve. Opening the bi-stable electro-magnetic angle valve causes a sudden and uncontrolled deposition of sample material onto the QCM and the sample sensor. To protect the sample sensor against this uncontrolled deposition, an electro-mechanically driven shutter, consisting of a small motor taken from an off-the-shelf CD-ROM drive and a PTFE plate, shields the sample sensor until controlled deposition is achieved.

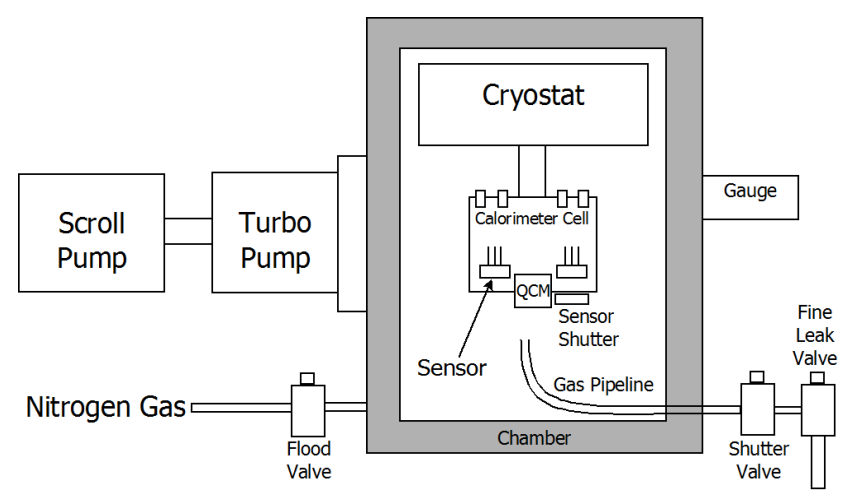

Figure 11: Vacuum setup with the heating/cooling and the deposition system. For simplification the liquid nitrogen containing dewar is drawn inside the chamber. In reality the dewar is connected via a $1 \mathrm{~m}$ long insulated PTFE pipe.

After constant deposition rate is achieved, the sensor shutter is opened to allow deposition onto the sample sensor. As soon as a predefined amount of material is deposited the bi-stable electro-magnetic angle valve and the sensor shutter are closed. The sensor shutter remains closed during the measurements to avoid radiative heating of the sensor membrane from the chamber walls being at room temperature.

\subsubsection{Sample holder}

A copper block (Figure 12 and Figure 13) acts as the heat sink and contains the sensors mounted in sockets, a Pt100 resistive thermometer, the cooling spiral and six cartridge heaters. The block is welded to the cooling spiral, which is in turn welded to the cooling pipes of a CF40 feedthrough flange. Cooling is done by liquid nitrogen. Several attempts to economize the liquid nitrogen consumption and to reach temperatures close to the liquid nitrogen temperature of about $77 \mathrm{~K}$ were done but welding a spiral to the block was the best choice. The six cartridge heaters 
$(6.5 \times 40 \mathrm{~mm}, 200 \mathrm{~W})$, connected in parallel, are set into the copper block using vacuum proof thermal grease for better heat transfer.

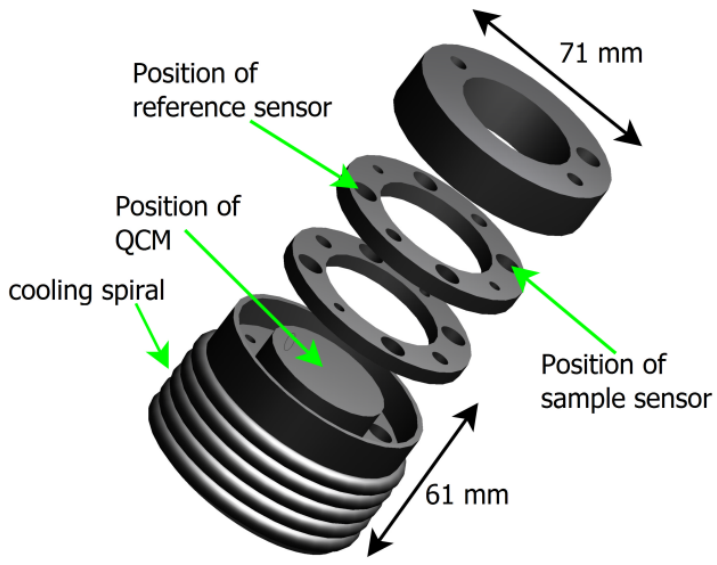

Figure 12: CAD drawing of the copper parts of the sample holder. The pipe for cooling the block with liquid nitrogen is welded spirally around the copper block. Two copper rings can be placed inside the notch of the copper block to fix the sockets and sensors. The upper ring is fixed on top of the block and shields the reference sensor from deposition.

The schematic of the block is shown in Figure 12. Copper rings are used to shield the reference sensor from deposition, to bring the sensors into the right position and to mount the shutter (Figure 13).

Installation of the sensors is critical and the cell construction aims at simplifying this. However, the long, fragile pins of the sensors have to be supported by insulating parts since the pins can easily bend and thus lose contact to the socket or can short-cut with another pin. To avoid this, small PTFE cylinders with 10 small passing holes are used to support each of the sensors legs (Figure 14). These cylinders perfectly fit into the holes of the copper rings.

The sensors are mounted in sockets. At the rear side the socket-pins are connected to PTFE insulated wires that lead directly to the vacuum side of the Sub-D 25 electrical feedthrough. Radial PTFE spacers are used to protect the pins of the sockets from short cuts with other pins or with the copper block (Figure 14). 

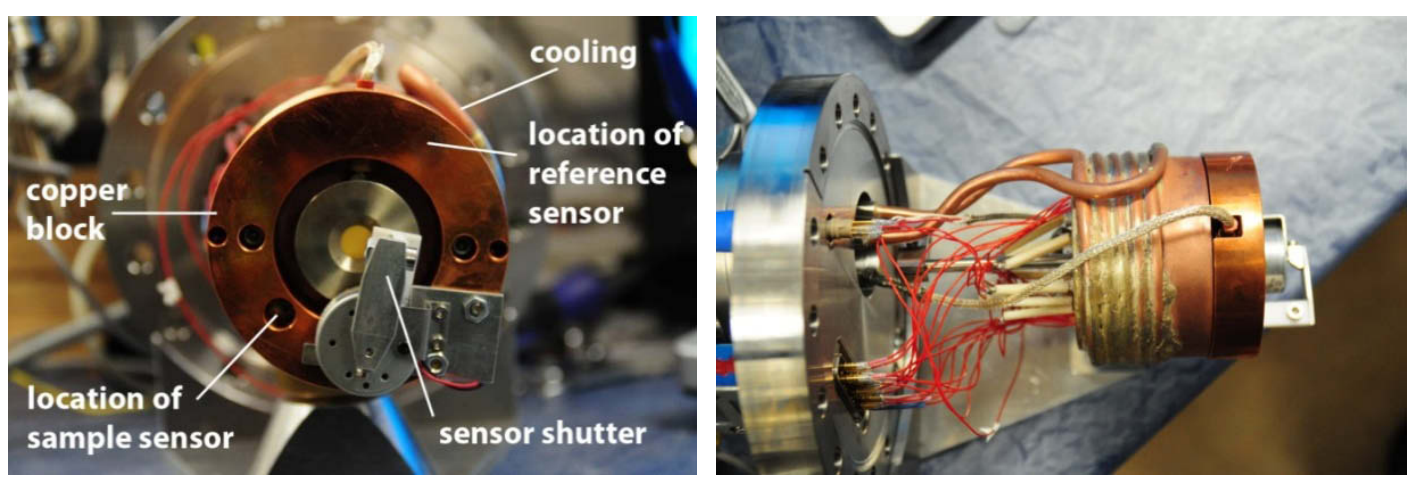

Figure 13: Left: The copper block with the cooling tube and the electro-mechanical shutter for shielding the sample sensor from uncontrolled deposition. The reference sensor is located opposite of the sample sensor fully covered by copper. Right: Copper block from top with the cooling spiral and the wiring of the sensors as well as of the cartridge heaters which are connected in parallel inside the chamber. The silver cable coming from the notch in the upper ring is connecting the QCM. A bolt Pt100 resistive thermometer is screwed into the center of the rear side of the sample holder and wired to the 10-pin feedthrough along with the sensor shutter and the cartridge heaters. The sensors are wired to the Sub-D 25 feedthrough.

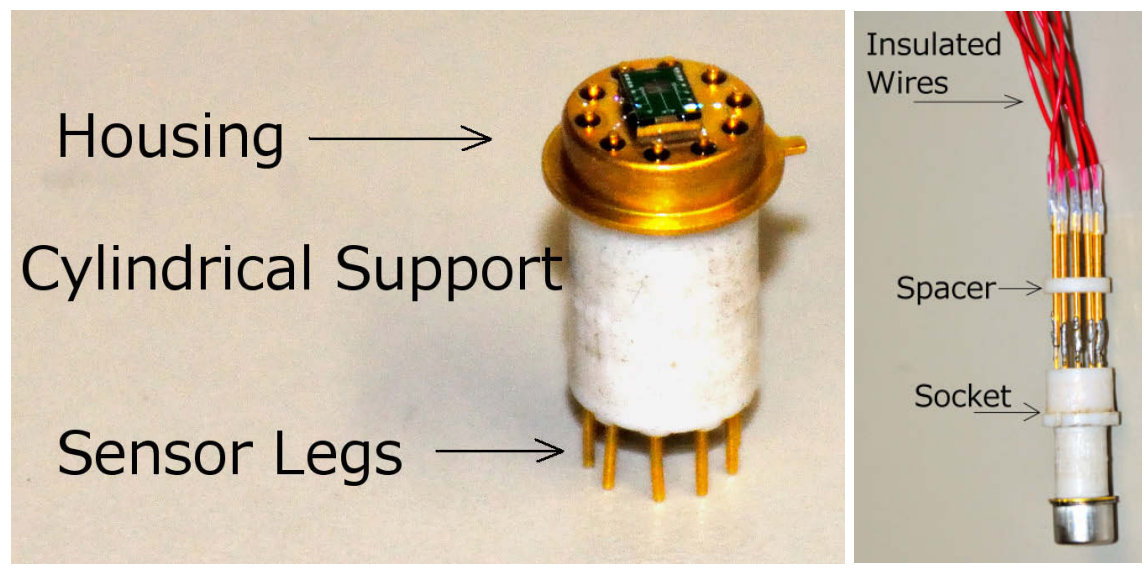

Figure 14: Left: Sensor with the PTFE support of the pins. Right: complete connection setup of the sensors with the socket, pins and cables leading to the Sub-D 25 electrical feedthrough.

Vacuum grease is used to increase the thermal contact between the copper parts, the sensor housing and the PTFE cylinders since vacuum between the parts can reduce the thermal coupling drastically.

The cooling of the copper block, accommodating the sensors, is enabled by a copper tube soldered to the outer wall of the block. This tube is connected via PTFE pipes to a liquid nitrogen 
dewar at $250 \mathrm{kPa}$. The flow of the liquid nitrogen is controlled by a magnetic valve at the dewar. Heating and cooling the copper block is realized by an Omron E5CK temperature controller. The electrical setup is shown in detail in the Appendix A. A resistive temperature device (RTD) (Pt100) is screwed into the center of the rear side of the copper block, opposite of the QCM. It is surrounded by the six cartridge heaters countersunk inside the block. The controller provides power to the heaters or opens the magnetic valve as needed.

The massive copper block smoothes out temperature oscillations coming from the temperature control. The PID parameters of the temperature controller were set to eliminate oscillations on a larger timescale but small oscillations at low temperatures may occur due to the $1 \mathrm{~m}$ long thermally insulated pipe from the dewar to the chamber. The massive block limits controllable heating rates to a maximum of $1 \mathrm{~K} \mathrm{~min}^{-1}$.

The system is limited to about $94 \mathrm{~K}$ at cryogenic temperatures due to the thermal contact to the top flange, radiative heat transfer from the chamber walls, and non-ideal insulation of the leading nitrogen. The maximum temperature is limited to approx. $520 \mathrm{~K}$ by the PTFE insulation inside the copper block.

\subsubsection{Migration to helium-cooled device}

Recently a $2^{\text {nd }}$ setup, similar to the one described above, was built using helium as the coolant (closed cycle helium cryostat from Cold-Edge Technologies, USA). Moreover some improvements in the construction of the device were made to enable higher heating/cooling rates. Unfortunately due to lack of space a QCM is not part of the new device. The helium-cooled device allows vapor deposition down to temperatures of $10 \mathrm{~K}$. The installed RTD can be used for temperature determination reliably and thus the heater resistance is not needed for temperature determination. The reliability of the temperature measurement was verified by measuring the glass transition temperatures of toluene and ethylbenzene, which were found to be about $3 \mathrm{~K}$ off to the expected value. This offset is highly reproducible from one temperature scan to the subsequent and thus the data can be corrected regarding the phase peak of the $20 \mathrm{~Hz}$ glass transition temperature of the liquid-cooled glass. The hysteresis of this device at a heating/cooling rate of $0.67 \mathrm{~K} \mathrm{~min}^{-1}$ gives a temperature difference between the temperature of the membrane of the sensor and the RTD temperature of only $0.2 \mathrm{~K}$.

In this new setup cooling is always on and the temperature is controlled by heating. Due to this the new copper block does not have to act as a thermal low pass and can be small. The He- 
cooled device is used for additional measurements whenever the temperature range of the LN2cooled device is not sufficient.

\subsection{AC calorimetry}

\subsubsection{Fundamentals}

Many different techniques have been developed to measure the heat capacity of materials. Generally two types of measurements can be performed: (i) scanning with a constant or variable rate or (ii) with periodic temperature oscillations. The latter, called AC calorimetry, can be straight forwardly linked to the dynamics of the molecular processes under investigation (e.g. dynamic glass transition, structural relaxation) while linear scanning provides information about freezing-in of the glass and allows determination of the enthalpy.

Corbino 1910 [73] first showed that the temperature oscillation caused by a periodically applied power to the sample is linked to its heat capacity. Subsequently this is demonstrated following Kraftmakher [74]. Corbino applied a power $p$ modulated by a sine wave to the sample

$$
p=p_{0}+\hat{p} \sin \omega t
$$

where $\hat{p}$ is the power amplitude, $p_{0}$ is the DC power and $\omega$ is the corresponding angular modulation frequency. The heat equation is balanced if the heat input equals the heat accumulated in the sample plus the heat losses

$$
\left(p_{0}+\hat{p} \sin \omega t\right) \Delta t=m c \Delta T+P(T) \Delta t
$$

where $m$ is the mass, $c$ is the specific heat capacity, $T$ is the temperature of the sample and $P(T)$ is the power corresponding to the heat losses from the sample. Equation (6) is formulated for a short time interval and thus $\Delta T$ is the change in temperature during this time $\Delta t$. If $T=T_{\text {avg }}+\theta$, where $T_{\text {avg }}$ is the mean sample temperature and $\theta$ is the modulated temperature oscillation, with $\theta \ll T_{\text {avg, }}$, $P(T)$ can be split into $P(T)=P\left(T_{a v g}\right)+P^{\prime} \theta$ with $P^{\prime}=\frac{d P}{d T}$ the heat transfer coefficient. This leads to

$$
m c \theta^{\prime}+P\left(T_{\text {avg }}\right)+P^{\prime} \theta=p_{0}+\hat{p} \sin \omega t
$$

with $\theta^{\prime}=\frac{d \theta}{d t}$. The steady-state solution of (7) is

$$
\begin{gathered}
P\left(T_{\text {avg }}\right)=p_{0} \\
\theta=\theta_{0} \sin (\omega t-\phi)
\end{gathered}
$$




$$
\begin{gathered}
\theta_{0}=\left(\frac{\hat{p}}{m c \omega}\right) \sin \phi=\frac{\hat{p} \cos \phi}{P^{\prime}}=\frac{\hat{p}}{\left(m^{2} c^{2} \omega^{2}+P^{\prime 2}\right)^{1 / 2}} \\
\tan \phi=\frac{m c \omega}{P^{\prime}}
\end{gathered}
$$

If the oscillations of the heat losses due to temperature oscillations $\left(P^{\prime}\right)$ in the sample are much smaller than the oscillations of the heating power $\hat{p}$ (the heat transfer coefficient is small) and the frequency is not too small the system can be regarded as quasiadiabatic which means $\tan \phi$ $\gg 1\left(\phi \approx 90^{\circ}\right)$. Thus $m c \omega \gg P^{\prime}$ and $(10)$ simplifies to

$$
m c=\frac{\hat{p}}{\omega \theta_{0}} .
$$

One can set a somewhat arbitrary limit as the beginning of the non-adiabatic regime. Kraftmakher set this limit to $\tan \phi<10$ and with (10) the non-adiabatic relation follows as

$$
m c=\frac{\hat{p}}{\omega \theta_{0}} \sin \phi
$$

Corbino [73] used the sample as a heater and thermometer simultaneously. If this is not the case, i.e. the thermometer and heaters are separate, one has to take thermal couplings between the sample (heat capacity $C_{s}$, temperature $\left.T_{s}\right)$, thermometer $\left(C_{t}, T_{t}\right)$, heater $\left(C_{h}, T_{h}\right)$ and the thermal conductivity of the sample into account (see Figure 15). This was first investigated by Kraftmakher 1962 [75] and Sullivan and Seidel in 1966 [76]. They used a bath of temperature $T_{b}$ as a heat sink which makes it necessary to add an additional thermal coupling between bath and sample.

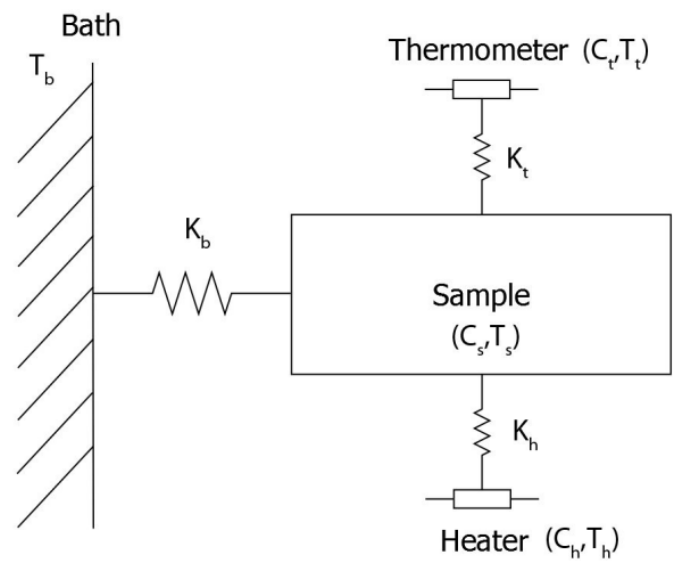

Figure 15: Schematic sketch of the setup investigated by Sullivan and Seidel [77]. The single parts are interconnected with the thermal links $K$.

Then the resulting equation for the temperature amplitude is: 


$$
\theta_{0}=\frac{\hat{p}}{\omega C \sqrt{1+\frac{1}{\omega^{2} \tau_{s}^{2}}+\omega^{2} \tau^{2}}}
$$

with $\tau_{s}$ the sample-to-bath relaxation time and $\tau$ the relaxation time of the sample. This equation is valid under the following conditions [77]:

(1) The heat capacity of the heaters and thermometer are much smaller than the heat capacity of the sample and can be neglected

(2) The sample, heater and thermometer equilibrate in a time much shorter than the modulation period

(3) The sample-to-bath relaxation time $\tau_{s}$ is much longer than the modulation period.

Regarding equation (14) quasi-adiabaticity is reached if the sum in the square root equals unity or

$$
\omega^{2} \tau_{s}^{2} \gg 1 \gg \omega^{2} \tau^{2}
$$

which means the external relaxation time characterized by $K_{b}$ must be larger than the square sum of the internal relaxation times coming from the heaters, thermometer and the sample. As a rule of thumb, $\tau_{s}$ should be two to three orders of magnitude lager than $\tau$. Thus, knowing the relaxation times of the system, it is necessary to choose the working frequency properly to ensure being in the quasiadiabatic regime. If equation (14) is multiplied by $\omega$ the right side of the resulting equation should be independent of frequency if adiabaticity is reached, resulting in a plateau in a $\omega \theta_{0}$ over $\omega$ plot (see Figure 16). $\omega \theta_{0}$ is often denoted as the transfer function. For low frequencies heat losses dominate and adiabaticity is not satisfied. For high frequencies the transfer function decreases due to the fact that the heater-sample-thermometer-system is not able to follow the high-frequency temperature modulation any more. 


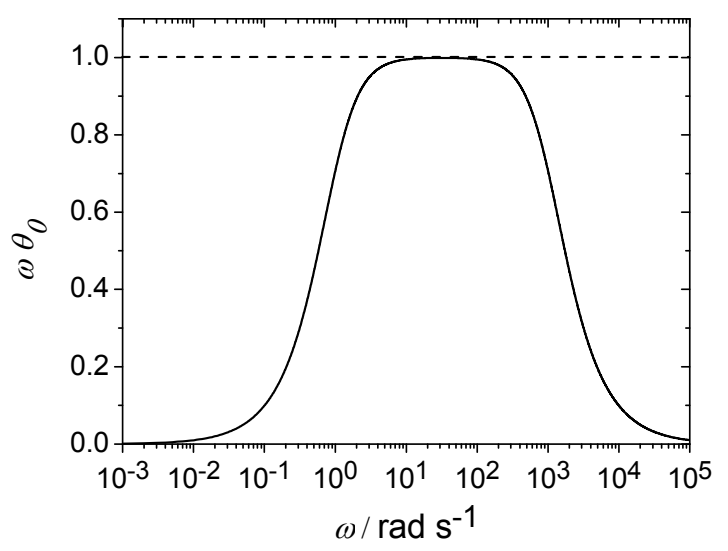

Figure 16: The transfer function following equation (10) multiplied with $\omega$. The heat capacity $C$ as well as the power amplitude $\hat{p}$ is set to unity. The sample-to-bath relaxation time $\tau_{s}$ is $1 \mathrm{~s}$, the relaxation time $\tau$ is $0.001 \mathrm{~s}$ and thus three orders of magnitude lower than $\tau_{s}$. The dashed line in the picture represents the maximum value of the transfer function at which adiabaticity is reached. Here the working frequency range spans one order of magnitude.

However, the sample masses needed to perform precise measurements with the method proposed by Sullivan and Seidel have to be sufficiently large, usually on the order of a few grams. This technique was improved by Velichkov [78] who supported the sample by a membrane (see Figure 17).

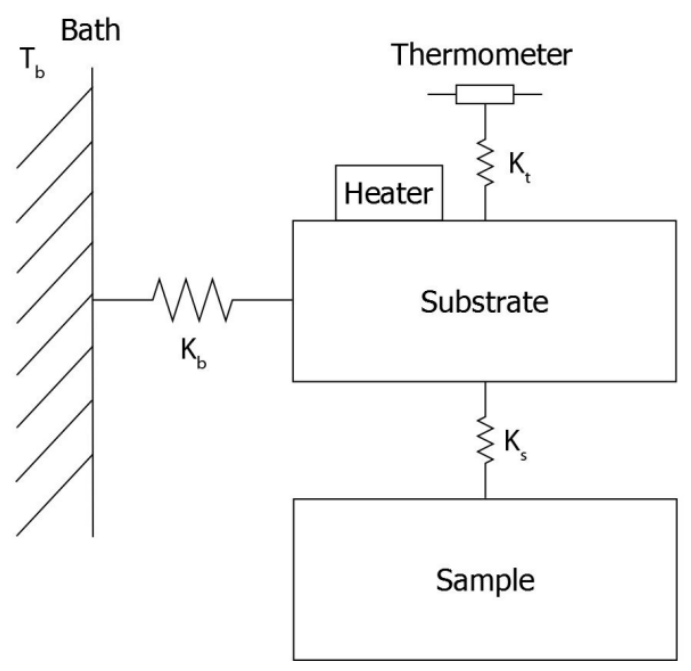

Figure 17: Schematic sketch of the setup as investigated by Velichkov [78]. The heater is perfectly attached to the substrate, enabling no thermal resistance between the two and also a very small heat capacity which makes the relaxation time of the heater negligible. 
Here the heater is coupled to the membrane in the form of a deposited thin layer of high resistivity material in good thermal contact with the substrate and of negligible heat capacity. For the investigation of this construction again it is necessary to assume the given quantities $C_{0}$ (heat capacity of the substrate), $C_{t}, C_{s}, K_{b}, K_{t}$ and $K_{s}$ to be constant within the temperature oscillation. Thus the temperature oscillation has to be small and is given by the complicated expression

$$
\begin{gathered}
\theta_{0}=\frac{\hat{p} \sqrt{\left(1+\omega^{2} \tau_{s}^{2}\right)}}{K_{b} \sqrt{\left(1-\omega^{2} a\right)^{2}+\omega^{2} b^{2}}} \\
\phi=-\arccos \left(( 1 - \omega ^ { 2 } a + \omega ^ { 2 } \tau _ { s } b ) \left(\left(1-\omega^{2} a+\omega^{2} \tau_{s} b\right)^{2}+\cdots\right.\right. \\
\left.\left.+\omega^{2}\left(b-\tau_{s}+\omega^{2} \tau_{s} a\right)^{2}\right)^{-\frac{1}{2}}\right)
\end{gathered}
$$

where

$$
\begin{gathered}
a=\tau_{b} \tau_{t}+\tau_{b} \tau_{s}+\tau_{s} \tau_{t}+\frac{\tau_{t} C_{s}}{K_{b}}+\frac{\tau_{s} C_{t}}{K_{b}} \\
b=\tau_{b}+\tau_{t}+\tau_{s}+\frac{C_{s}}{K_{b}}+\frac{C_{t}}{K_{b}}-\omega^{2} \tau_{b} \tau_{t} \tau_{s}
\end{gathered}
$$

and $\tau_{b}=C_{0} / K_{b}, \tau_{t}=C_{t} / K_{t}, \tau_{s}=C_{s} / K_{s}$.

Here the single heat capacities involved in the setup are separated and knowledge of the heat capacities of the thermometer and the substrate makes it possible to determine the heat capacity of the sample. Because the solution of Sullivan and Seidel included the heat capacities of the sample, thermometer and the heater in a single value it was necessary to take relatively high sample masses for the measurement. The solution provided by Velichkov enables to use much smaller samples, even smaller than the heat capacities of the substrate or the thermometer. For the analytical investigation of the complicated expression above it is advantageous to simplify the equation by making some assumptions. Here we want to consider a calorimeter with a sample and a perfectly attached thermometer $\left(K_{t} \rightarrow \infty, 1 . K_{b}=K_{s}, 2 . K_{b}=10^{3} K_{s}\right)$ as well as a thermally well attached thermometer $K_{t}=10^{4} K_{b}=10 K_{s}$ and balanced heat capacities $C_{s} \approx C_{0} \approx C_{t}$. This gives

$$
\theta_{0}=\frac{\hat{p} \sqrt{\left(1+\omega^{2} \tau_{b}^{2}\right)}}{K_{b} \sqrt{\left(1-2 \omega^{2} \tau_{b}^{2}\right)^{2}+16 \omega^{2} \tau_{b}^{2}}}
$$

for the case $K_{t} \rightarrow \infty, K_{b}=K_{s}$ and the corresponding transfer function is plotted as the black line in Figure 18. The heat capacities are set to $C_{0}$ and $K_{b}$ is set to unity. The transfer function reaches the adiabatic plateau with a value of $1 / 2$ (due to the poorly attached sample) for frequencies above 
$20 \mathrm{rad} \mathrm{s}^{-1}$. In this case the transfer function does not decrease for higher frequencies due to the perfectly attached thermometer.

The second case $K_{t} \rightarrow \infty$ and $K_{b}=10^{3} K_{s}$ leads to the following equation for the modulated temperature amplitude

$$
\theta_{0}=\frac{\hat{p} \sqrt{\left(1+1 \cdot 10^{-6} \omega^{2} \tau_{b}^{2}\right)}}{K_{b} \sqrt{\left(1-2 \cdot 10^{-3} \omega^{2} \tau_{b}^{2}\right)^{2}+\left(3+1 \cdot 10^{-3}\right)^{2} \omega^{2} \tau_{b}^{2}}}
$$

This case is represented by the red line in Figure 18. Since now $K_{b}$ is much higher than $K_{s}$ the function becomes two-stage. After reaching the first plateau at about $5 \mathrm{rad} \mathrm{s}^{-1}$ all heat capacities equally contribute to the transfer function which leads to the value of $1 / 3$. At about 100 $\mathrm{rad} \mathrm{s}^{-1}$ the function starts to increase since the sample starts to lag behind the temperature oscillations and does not affect the transfer function at all. The infinite thermal conductivity of the thermometer is again responsible for the constant transfer function above $10^{4} \mathrm{rad} \mathrm{s}^{-1}$.

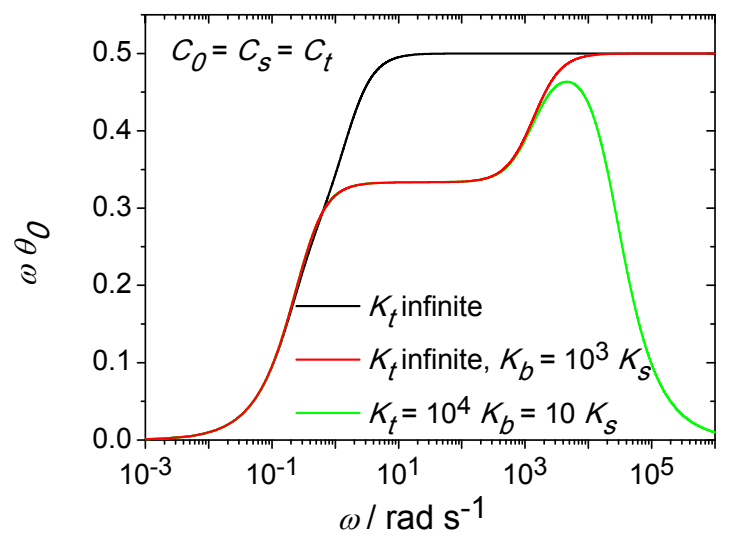

Figure 18: Three different solutions for the transfer function as calculated from the model proposed by Velichkov.

The third case $K_{t}=10^{4} K_{b}=10 K_{s}$ represented by the green line in gives

$$
\theta_{0}=\frac{\hat{p} \sqrt{\left(1+1 \cdot 10^{-6} \omega^{2} \tau_{b}^{2}\right)}}{K_{b} \sqrt{\left(1-2.2 \cdot 10^{-3} \omega^{2} \tau_{b}^{2}\right)^{2}+\omega^{2}\left(3 \tau_{b}-1 \cdot 10^{-7} \omega^{2} \tau_{b}^{3}\right)^{2}}}
$$

Up to about $1000 \mathrm{rad} \mathrm{s}^{-1}$ the transfer function follows the previous case but then the finite thermal link of the thermometer causes the transfer function to decrease as the thermometer starts to lag behind the temperature oscillations. This case represents the real case since the thermal link of the thermometer must have a finite conductivity.

This model still has the problem that the thermal diffusivity of the sample and the membrane are not included. Greene et al. [79] and later Riou et al. [80] extended the model of 
Velichkov in this respect. Greene et al. investigated the case of a sample suspended by wire thermocouples of length $\lambda$. The diffusive contribution of a thermocouple wire can be expressed by the functional

$$
F(x)=\frac{1}{x} \frac{\sinh x-\sin x}{\cosh x-\cos x}
$$

with $x=2 k(\omega) \times \lambda$ and $k$ the diffusive wavevector. Recently Rydh et al. extended this model assuming not only the contribution of the heat capacity of the substrate to be frequency dependent but also the thermal conductivity of the substrate. Furthermore a frequency dependent effective link to the sample was introduced to the model. Heater and thermometer are assumed to be perfectly attached to the substrate. This leads to the following equation for the modulated temperature amplitude and phase

$$
\begin{gathered}
\theta_{0}=\frac{\hat{p}}{\sqrt{\left(\omega\left(C_{h}+C_{t}+C_{0, e f f}+(1-g) C_{s}\right)\right)^{2}+\left(K_{b, e f f}+g K_{s}\right)^{2}}} \\
\tan \phi=\frac{\omega\left(C_{h}+C_{t}+C_{0, e f f}+(1-g) C_{s}\right)}{K_{b, e f f}+g K_{s}}
\end{gathered}
$$

with

$$
\begin{gathered}
C_{0, e f f}=\frac{C_{0}}{\alpha} \frac{\sinh \alpha-\sin \alpha}{\cosh \alpha-\cos \alpha} \\
K_{b, e f f}=\frac{K_{b} \alpha}{2} \frac{\sinh \alpha+\sin \alpha}{\cosh \alpha-\cos \alpha} \\
g=\frac{\left(\omega \tau_{t}\right)^{2}}{1+\left(\omega \tau_{t}\right)^{2}}
\end{gathered}
$$

and $\alpha=\sqrt{2 \omega \tau_{b}} \cdot C_{h}$ is the heat capacity of the heater. Figure 19 shows the transfer function of this solution assuming the same values for the heat capacities and thermal conductivities as mentioned above and $K_{t} \rightarrow \infty$ as heater and thermometer are perfectly attached to the substrate. Additionally $C_{s} \approx C_{0} \approx C_{t} \approx C_{h}$ shall hold. Compared to the solution of the model proposed by Velichkov the transfer function is shifted to higher frequencies. The parameter $g$ can be used to determine the working frequency and should be small to obtain good accuracy in the heat capacity determination. 


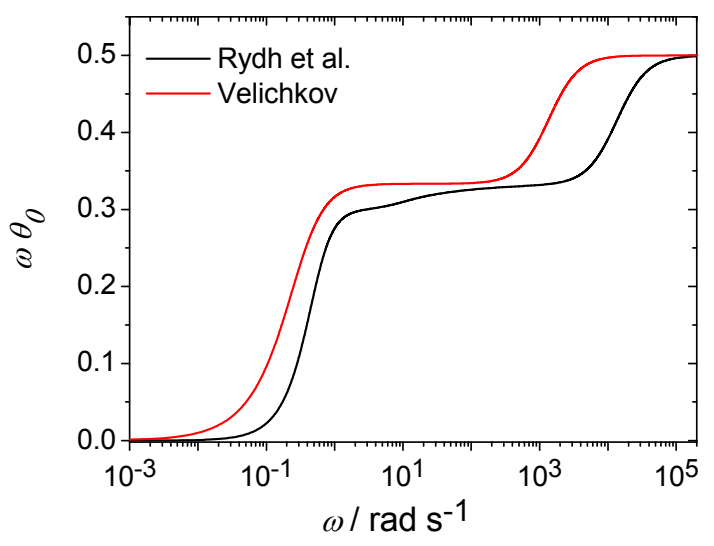

Figure 19: The transfer function of a membrane based calorimeter cell, represented by the black line, taking into account the thermal diffusivity of the substrate and the effective thermal link to the sample. The red line represents the corresponding transfer function of the model proposed by Velichkov.

Knowing the parameters, which can often be measured, this technique gives the heat capacity with good accuracy. It is now widely used to determine heat capacities of small samples [81-84].

Different from the considerations of Sullivan and Seidel where the sample is in direct contact to the bath and thermometers we use a membrane suspended system. Our samples are deposited onto $\mathrm{SiO}_{2}$-membranes. This means the sample has no direct contact to the bath and thermometers anymore and thus the relaxation time of the sample $\tau$ of Sullivan and Seidel's system has to be regarded now as the internal time constant of the sensor. The sample-to-bath relaxation time $\tau_{s}$ has to be regarded now as the external time constant of the sensor. Figure 20 shows the transfer function of a sensor equivalent to the used sensors (see next chapter) in vacuum and ambient pressure. Obviously an adiabatic plateau is absent which indicates all considered analytical models which presume adiabaticity are not applicable for the used sensors. This is due to the fact that the internal and external time constant are too close to each other. Measurements yielded that the external and internal time constants are separated by only one order of magnitude. Whether the peak value at about $f_{\text {th }}=2 \mathrm{kHz}$ in Figure 20 is showing quasi-adiabaticity or if it is way off of it cannot be proven and thus the working frequency of qualitative experiments is chosen with regards to other aspects. This means direct measurement of the absolute heat capacity is not possible with the analytical descriptions mentioned above.

For the kind of sensors used for this work Minakov et al. designed a model for absolute heat capacity determination via AC calorimetry in a gas surrounding $[85,86]$ on the basis of the 
work of Merzlyakov [87]. The fundamental formula (12) of AC calorimetry in the complex plane is

$$
C_{a p}=\frac{\hat{p}}{i \omega \theta_{0}}
$$

with $C_{a p}$ as the apparent heat capacity. The fact that the chip calorimeter is driven in a gas surrounding leads to a term taking into account the dominant heat losses through the gas. Lateral heat losses are neglected since this is minor compared to the losses through the gas.

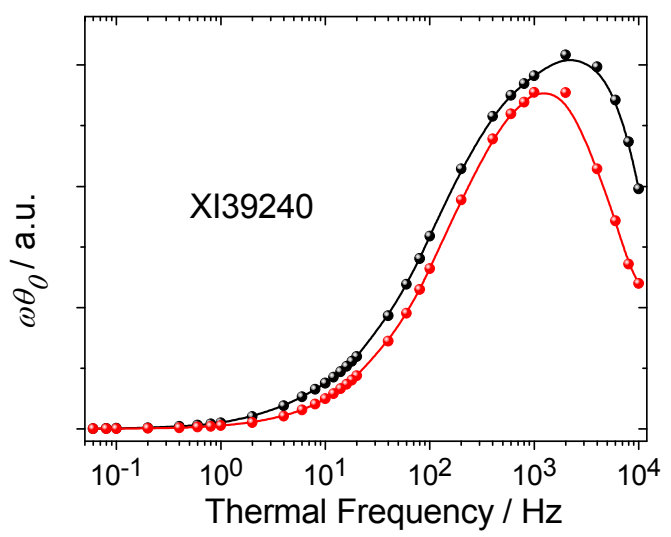

Figure 20: Transfer function of the sensor XI39240 measured in ambient (red) and vacuum (black) condition.

The heater of the membrane is considered as a point source and then the heat wave can be described by a spherical wave $T_{\text {gas }}(t, r) \sim \exp \left(i \omega t-k_{\text {gas }} r\right) / r$ with $k_{\text {gas }}^{2}=i \omega \rho_{\text {gas }} c_{\text {gas }} / \lambda_{\text {gas }}, \lambda_{\text {gas }}$ the thermal conductivity, $c_{\text {gas }}$ the heat capacity and $\rho_{\text {gas }}$ the density of the surrounding gas. The heat loss through the gas can be described by the heat exchange coefficient $G=4 \pi r_{0} \lambda_{\text {gas }}$ with the small radius of the heated area $r_{0}$ compared to the lateral dimensions of the membrane. $G$ is considered to be frequency independent up to at least $1 \mathrm{kHz}$ [86]. The modulated temperature amplitude follows from the Fourier heat flux equation to

$$
\theta_{0}=\frac{\hat{p}}{(i \omega C+G)}
$$

with $C$ as the heat capacity of sample plus addenda. From (29) and (30) follows $C_{a p}=C+G / i \omega$. The heat capacity $C$ can be described as the apparent addenda heat capacity $C_{0}(\omega)=C_{00} \sqrt{\left(1+\frac{\omega_{0}}{i \omega}\right)}$ plus the effective heat capacity of the sample $\frac{C_{s} \tanh \alpha_{s}}{\alpha_{s}}$ with the effective thermal thickness of the sample $\alpha_{s}=d_{s} k_{s}$ and the finite sample thickness $d_{s}$ and $k_{s}$ as the thermal wave number of the sample. To take into account that the modulated temperature is not measured 
at the position of the heaters the effective thermal distance between heater and thermopiles is included by a factor $\cosh \left(\alpha_{0}\right)$. From isothermal frequency dependent measurements the parameters $\alpha_{0}, \omega_{0}, C_{00}$ and $G$ as well as the sensitivity $S$ of the thermopile can be obtained [86]. The factor $\tanh \alpha_{s} / \alpha_{s}$ equals one for thin films and the apparent heat capacity equals

$$
C_{a p}(\omega)=\cosh \alpha_{0}\left[C_{0}(\omega)+C_{s}+\frac{G}{i \omega}\right]
$$

Since for thin films the apparent addenda heat capacity $C_{0}$ dominates the use of a differential sensor setup minimizes the influence of the apparent addenda heat capacity and increases the sensitivity of AC calorimetric measurements [88]. The differential modulated temperature amplitude follows from (30) and (31) to

$$
\frac{i \omega \cosh \alpha_{0} \Delta \theta_{0}}{P_{0}}=\frac{1}{C_{0}(\omega)+C_{s}+\frac{G}{i \omega}}-\frac{1}{C_{0}(\omega)+\frac{G}{i \omega}} \cong \frac{C_{s}}{\left(C_{0}(\omega)+\frac{G}{i \omega}\right)^{2}}
$$

assuming $C_{s} \ll C_{00}$. In (32) two identical sensors are assumed. However, one sensor is never absolutely identical with another due to small differences in the membrane thickness or the composition of constituents of the chip sensor. The small difference can be taken into account by measuring the empty differential system which gives an additional term $\frac{\delta C_{0}(\omega)+\frac{\delta G}{i \omega}}{\left(C_{0}(\omega)+\frac{G}{i \omega}\right)^{2}}$ and with $\widetilde{C}_{s}=C_{0}(\omega)+\frac{G}{i \omega}$ follows the final equation for heat capacity determination under ambient pressure

$$
C_{s}=\frac{i \omega \cosh \alpha_{0} \tilde{C}_{s}^{2}\left(\Delta \theta_{0}-\Delta \theta_{00}\right)}{\hat{p}}
$$

Here the term $\Delta \theta_{0}-\Delta \theta_{00}$ is the difference between the differential complex temperature amplitudes of the loaded and the empty system. The measured differential thermopile amplitude can be converted to differential temperature amplitude by the thermopile sensitivity $S=1.3 \mathrm{mV} / \mathrm{K}$, see chapter 3.3.5.

With this method the commercial AC chip-nanocalorimeters now allow determination of the complex heat capacity of thin films down to a few $\mathrm{nm}[12,89-91]$. However, heat capacity determination is difficult since uncertainties in the determination of needed quantities, particularly $\widetilde{C}_{s}$, leads to high uncertainties in the sample heat capacity. Moreover, as one can imagine the absence of a surrounding gas lowers the heat losses drastically and only lateral heat flux is possible (radiation is assumed to have a minor effect). Thus the thermal resistance of the membrane is responsible for the heat transfer, but this is neglected in the model by Minakov et al. Thus this model is not applicable for UHV conditions. 
As opposed to heat exchange with a gas, the lateral heat exchange with the frame causes an increase in the temperature amplitude of a single sensor since the heat loss is reduced. From that a higher signal-to-noise ratio and thus higher resolution of the sensor is expected, which allows for the measurement of very thin film samples. Moreover the frequency dependence of the temperature amplitude is expected to change in a way to enlarge the working range.

The frequency dependent temperature amplitude was measured at room temperature for the case of a surrounding gas (air) at ambient pressure and in UHV (see Figure 21) using two different chip-calorimeters purchased by Xensor, NL. It can be seen that the temperature amplitude at the low frequency plateau is almost doubled changing to vacuum and the cut-off frequency (where the temperature amplitude starts to drop) is shifted about one order of magnitude to lower values. Interestingly the old sensor type TGC3880 (see inset in Figure 21) has a better ratio between heat loss through the gas and the membrane. However, recent changes in the membrane thickness and active area compared to the old sensor types increased the heat loss through the membrane drastically. Regardless, as can be seen from these measurements the role of lateral heat loss through the membrane in a surrounding gas cannot be neglected. Since this is done by Minakov et al. the derived sample heat capacities are overestimated.

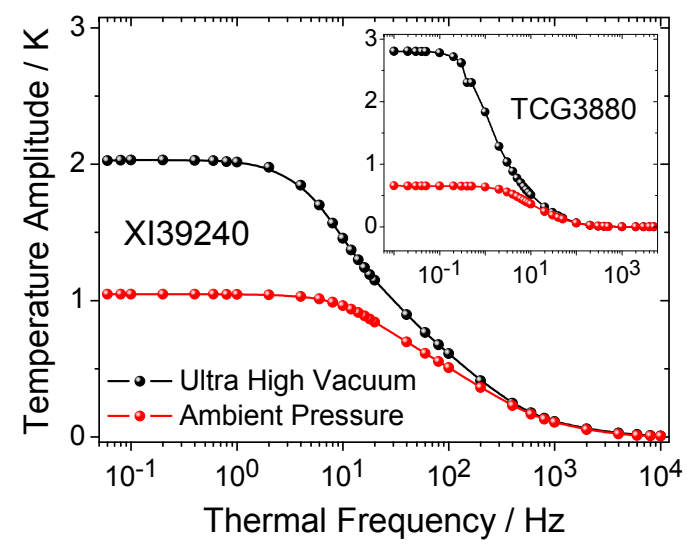

Figure 21: Single sensor temperature amplitude as a function of thermal frequency and pressure for two different sensor types. The larger area sensor TGC3880 shows a significantly higher difference in the temperature amplitude between ambient and vacuum conditions.

With the appearance of small addenda heat capacity sensors [92-95] which allowed the measurement of very small samples (down to $\mathrm{pg}$ ) it was possible to increase the frequency of temperature modulation of the quasiadiabatic state up to the $\mathrm{kHz}$ region $[11,85,89]$ as well as the heating rate which lead to Fast Scanning Calorimetry [94, 96-98]. 


\subsubsection{AC calorimeter}

The chip sensors XI39390 used in this device are commercially available nanocalorimeters from Xensor Integrations, NL [99]. The chip is based on a sub-micron thick $\mathrm{SiN}_{\mathrm{x}}$-membrane on which two pairs of parallel heater stripes of $5 \mu \mathrm{m}$ width, the inner and the outer heaters, are located in the very center and separated by $30 \mu \mathrm{m}$ and $40 \mu \mathrm{m}$, respectively (see Figure 22). The area between the heater stripes of $30 \mu \mathrm{m} \times 30 \mu \mathrm{m}$ shows an almost uniform temperature profile and most of the measured signal originates from this "active area" [100]. The six hot junctions of the thermopile are located in between the heaters; the cold junctions are placed far outside the active area on the silicon frame. The layer accommodating the heaters, thermopile and conducting stripes, is covered by an additional $700 \mathrm{~nm} \mathrm{SiO}_{2}$ layer for electrical and mechanical protection of the circuits.

The sensors made by Xensor are now widely used and thus the various types of sensors were tuned to several applications. In the case of the sensor XI39390 the sub-micron thick $\mathrm{SiN}_{\mathrm{x}}$ membrane exhibits a much higher thermal resistance between the middle of the membrane and the silicon frame compared to earlier mono-crystalline membranes used by Xensor. The high thermal resistance (about $28 \mathrm{kK} \mathrm{W}^{-1}$ at ambient temperature) enables the detection of much smaller heat effects, which is appropriate for this work.
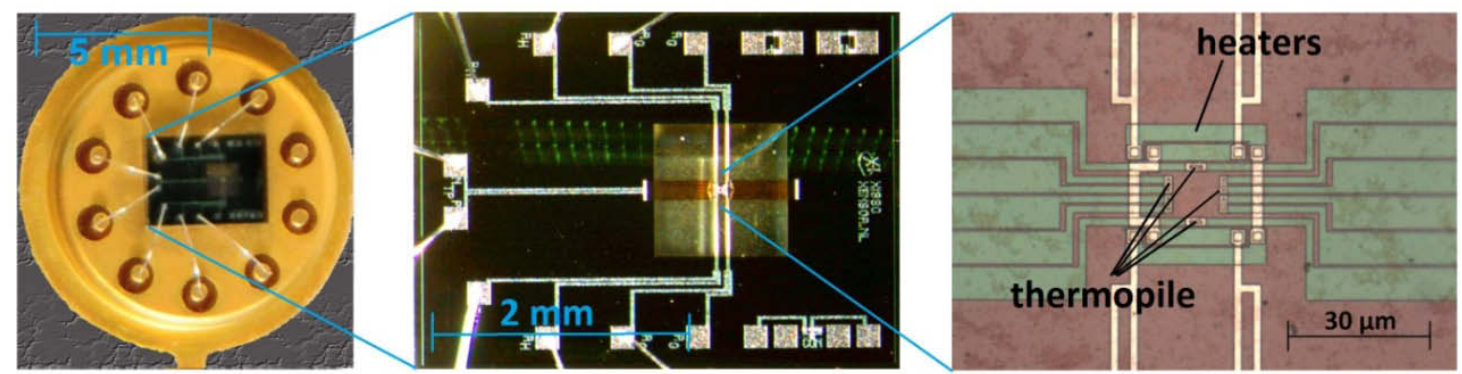

Figure 22: Photographs of the calorimetric chip sensor XI39390 at three different magnifications. Left: chip mounted on TO5 housing, middle: the chip, right: the central part, including the active area.

The electrical scheme of the AC calorimeter closely follows the device described by Huth et al. $[12,89]$. An applied power to the heaters of the sensor with an oscillator frequency $\omega$ causes a temperature oscillation at the heaters with a thermal frequency $f_{t h}$ of $2 \omega$. This phase shifted temperature oscillation is detected by the thermopiles, located in the active area of the membrane of the sensor. The complex amplitude of the thermopile signal, measured as the difference 
between the sample and reference sensors, depends on the sample heat capacity $\left(\mathrm{J} \mathrm{K}^{-1}\right)$ and the applied frequency. The magnitude and phase of the differential thermopile signal are measured with a lock-in amplifier SR7265 from Signal Recovery using the internal oscillator to apply oscillating power to the inner heater. This is converted to temperature amplitude using the thermopile sensitivity of $1.3 \mathrm{mV} \mathrm{K}^{-1}$. The magnitude equals the modulus $\sqrt{c^{\prime 2}+\left(-c^{\prime \prime}\right)^{2}}$ of the complex heat capacity $c=c^{\prime}-i c^{\prime \prime}$. The phase is calculated from $\arctan \left(\frac{-c^{\prime \prime}}{c^{\prime}}\right)$.

The oscillator output, the on membrane heaters of the sample and the reference sensors (each about $700 \Omega$ at room temperature), and a stable known resistor $R_{i}$ of $6790 \Omega$ are connected in series as shown in Figure 23. Usually $1 \mathrm{~V}_{\text {rms }}$ is applied to this circuit and thus only about 0.1 $\mathrm{V}_{\text {rms }}$ drops at the resistive membrane heaters. The voltage to the heaters has to be small to be sure the temperature amplitude remains small. Fragile glass formers like the ones used in this work exhibit a small temperature range $(\approx 5 \mathrm{~K})$ at the glass transition and a temperature amplitude of a few Kelvins would introduce uncertainties in the determination of the dynamic glass transition. To avoid this, the applied power at the heaters was set to $1 \cdot 10^{-5} \mathrm{~W}$ for all experiments, which resulted in a temperature amplitude of about $0.15 \mathrm{~K}$ at a frequency of $20 \mathrm{~Hz}$.

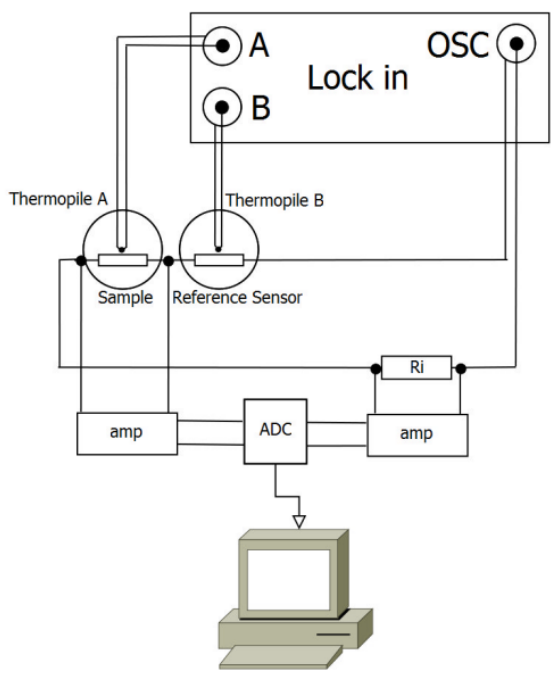

Figure 23: Scheme of the electronic setup of the differential AC calorimeter. The oscillating voltage of the lock-in amplifier drops at the two inner heaters of both chip sensors and the known resistor $R_{i}$, which are connected in series. The voltage drop at the inner heater of the sample sensor as well as at the reference resistor is amplified by instrumentation amplifiers (amp, AD620) and measured by an analog-to-digital converter (ADC). From these values the heater resistance and the applied power can be determined. The differential temperature amplitude is directly measured by the digital lock-in. 
The frequency giving the highest complex differential temperature amplitude is taken as the working frequency in all subsequent measurements. Figure 24 shows the frequency dependent complex differential temperature amplitude of an empty sensor pair as well as for the loaded sensor pair with a thin film of toluene at $T_{\text {substrate }}=105 \mathrm{~K}$. For the empty and the loaded frequency sweeps the maximum complex differential temperature amplitude is observed for a thermal frequency of $20 \mathrm{~Hz}$. Below that frequency the lateral heat loss to the bath lowers the thermopile signal, above the membrane plus sample cannot follow the oscillation and thus the heated part of the membrane and sample is reduced.

Figure 24 shows that the used sensors, if driven in UHV, can detect the temperature amplitude coming from the sample in a frequency window spanning five orders of magnitude.

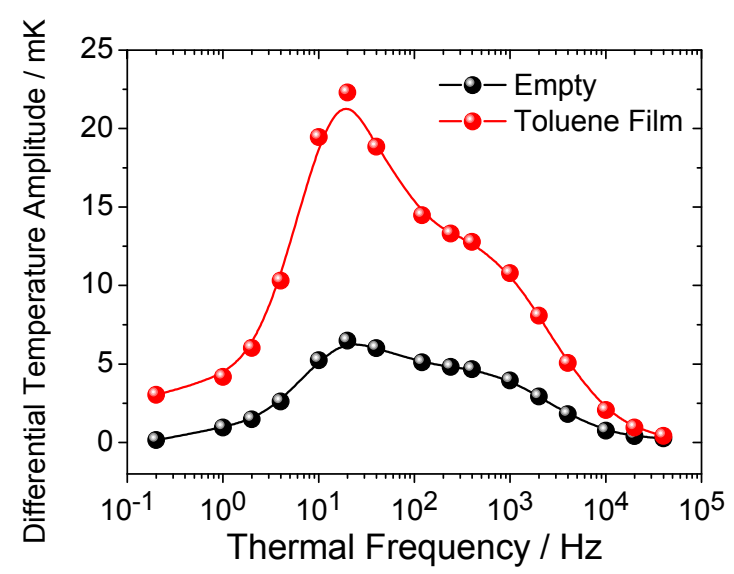

Figure 24: Differential temperature amplitude of the differential setup in vacuum and at $T_{\text {substrate }}=105 \mathrm{~K}$ as a function of frequency. The black data represents the empty system; the red data represent the data of a $390 \mathrm{~nm}$ thick film of toluene.

\subsubsection{Complex heat capacity}

The lock-in amplifier is set to measure the magnitude and phase of the thermopile response, which allows the determination of the apparent complex heat capacity (addenda + sample heat capacity). The differential setup compensates for the addenda heat capacity of the sensors except for a small asymmetry of the sensors. This asymmetry can be taken into account by subtracting a baseline (empty measurement).

At the glass transition the heat capacity increases. This shows up as a decrease of the temperature amplitude of the sample sensor. As a consequence, for a perfectly symmetric sensor pair, the differential temperature amplitude increases. The phase shows a minimum at the dynamic glass transition due to the relaxation process. This is not a contradiction to the maximum 
commonly seen in the loss function $c^{\prime \prime}$ at the glass transition from e.g. TMDSC measurements since the phase is $\arctan \left(\frac{c^{\prime \prime}}{c^{\prime}}\right)$ and thus not necessarily following $c^{\prime \prime}$.

The reference sensor gives a complex temperature amplitude that is a single point in the complex plane and is constant during e.g. the deposition process. By contrast the complex temperature amplitude of the sample sensor follows a trajectory during the deposition which can point in a random direction. The starting point and the direction of the trajectory depend on the sample sensor that is used. Figure 25 shows the complex temperature amplitude of both the reference and sample sensors in the complex plane during a deposition.
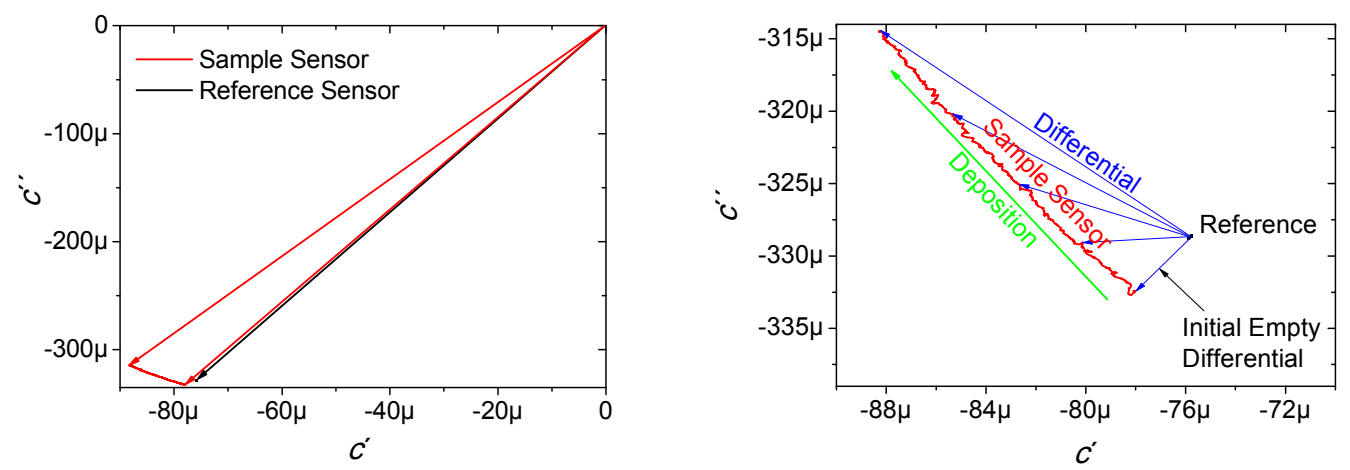

Figure 25: Complex temperature amplitude of the reference and sample sensors visualized in the complex plane (left: full scale, right: close-up). The trajectory (red line) of the sample sensor shows the evolution of the complex temperature amplitude during a common deposition of a thin toluene film onto the sample sensor (temporal evolution indicated by the green arrow). The reference sensor provides a single point in the complex plane. The blue arrows show some randomly chosen values of the complex differential temperature amplitude.

At the initial point the differential temperature amplitude represents the empty system. As the deposition precedes the complex differential temperature amplitude increases. Usually the evolution of the trajectory of the temperature amplitude of the sample sensor does not proceed parallel to the pointing vector of the temperature amplitude of the reference sensor (see left panel of Figure 25) thus the phase information has to be taken into account by baseline subtraction in the complex plane. Complex baseline subtraction is recommended otherwise the resulting data can be in error. Subtraction in the real plane yields the evolution of the temperature amplitude as shown in the right panel of Figure 26. The difference is obvious. The baseline corrected differential temperature amplitude calculated in the real plane is underestimated and thus the heat capacity is too. 

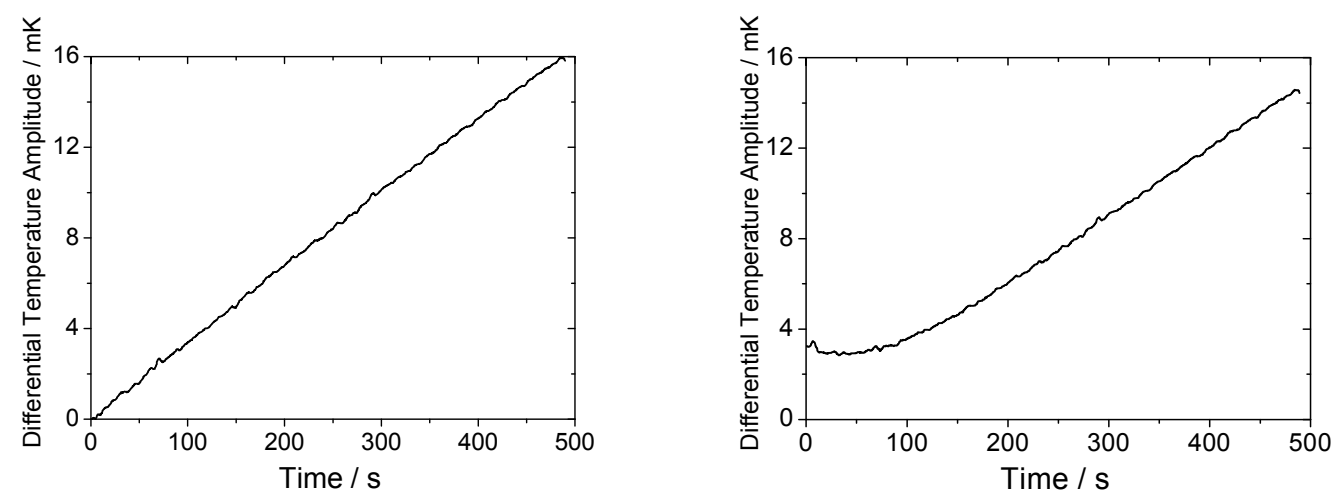

Figure 26: Evolution of the differential temperature amplitude during deposition of a thin toluene film onto the sample sensor, calculated from the values of the complex differential temperature amplitudes of the loaded and empty systems. The left panel shows the result of subtraction in the complex plane, the right panel of subtraction in the real plane.

\subsubsection{Heat capacity determination}

The next step, the determination of the heat capacity from the differential temperature amplitude, is challenging. Minakov et al. [86] analytically described an AC calorimeter based on a single thin film sensor similar to the sensors used here. The solution of the heat transfer problem assumes negligible heat losses through the membrane and dominating losses through the surrounding gas. Since our aim is the in-situ investigation of as-deposited glasses in vacuum, Minakov's model is not applicable here. In vacuum the membrane provides the only heat loss path, thus the membrane geometry determines the heat flow. Efforts to describe the sensor with a simple analytical model, following references $[78,80]$, failed as the structure of the sensor seems to be too complicated. Additionally a semi-analytical approach considering the membrane as a series of electrical low passes with thermal resistances treated as electrical resistances and heat capacities treated as electrical capacitances (see Figure 28) lead to no success. That model can be described as a "large elements model" since the membrane is divided into only 16 rings each of them having individual thermal resistances and heat capacities (Figure 27). 

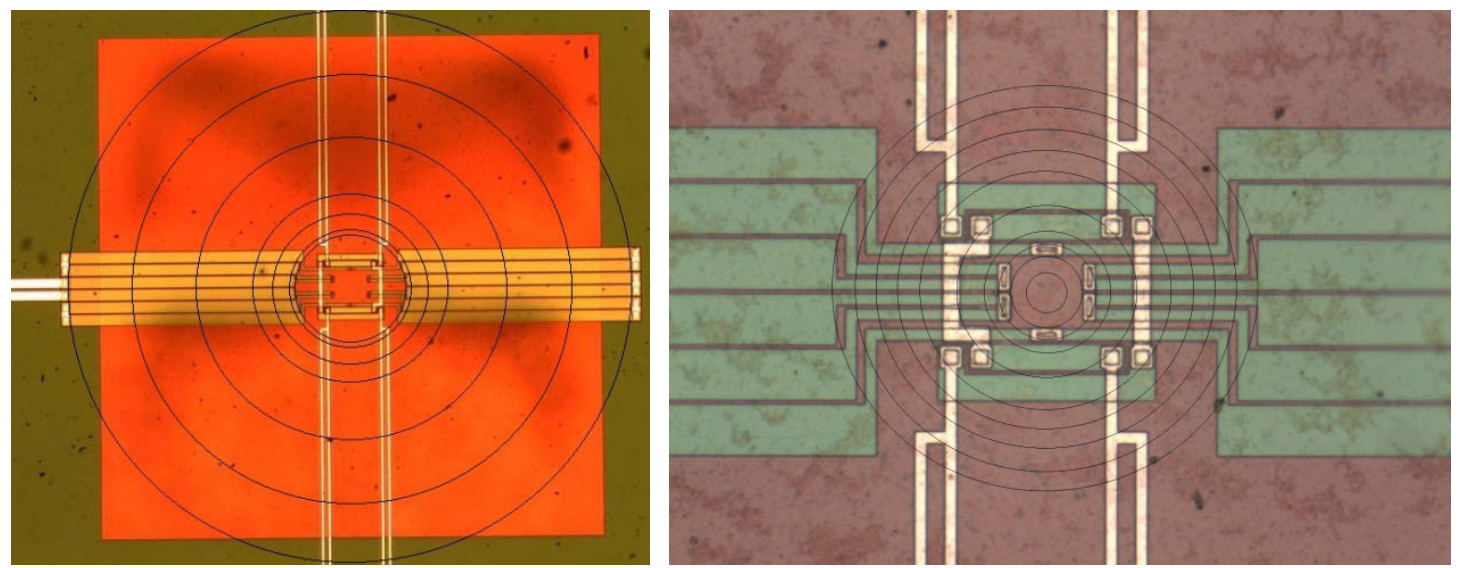

Figure 27: Splitting of the membrane of the sensors into 16 rings (left: six outer; right: 10 inner rings).

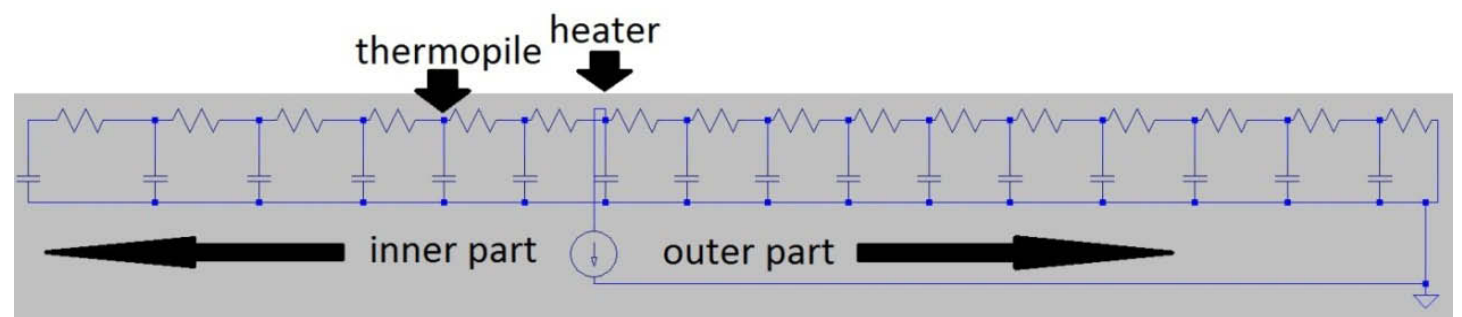

Figure 28: Schematic picture of the semi-analytical model dividing the membrane into 16 electrical low passes. The location of the heater stripes and the thermopiles are shown. The power is supplied to the heaters and the ground (bath).

The membrane of the sensor is traversed by conducting stripes coming from the heaters and the thermopiles, which makes it necessary to divide the membrane carefully regarding its internal geometry. The 16 rings can be divided into 6 rings for describing the inner part of the membrane and 10 rings for the outer part beyond the heater stripes. The radii of the rings are not equidistant. The overall thermal resistance of the membrane can be determined from the low frequency regime temperature amplitude and the applied power. The overall thermal resistance of the membrane treated as electrical resistance is $71160 \Omega$. This value has to be split reasonably to the 16 low passes. The inner part of the membrane shows a homogeneous temperature distribution for the DC case [100] which allows for the assumption of constant thermal resistance per unit volume. The electrical resistance for the outer rings decreases like $1 / r$ with $r$ being the radius vector. The capacitance (heat capacity) of the rings is hard to calculate using the heat capacity of the $\mathrm{SiN}_{\mathrm{x}}$ and the geometry of the rings since the additional parts of the membrane have unknown contributing fractions and unknown heat capacities. Thus it is challenging to find values for the capacitances and the thermal resistances for each of the rings. Frequency dependent empty and 
loaded single sensor measurements as well as differential measurements were used for fitting but satisfactory solutions could not be found. Hence a finite elements model (FEM) was designed by Evgeni Shoifet, a member of the Polymer Physics group of the University of Rostock [13].

The numerical model is based upon the detailed geometrical description of the calorimetric sensor XI39390. The sensor consists of a silicon frame and a free standing $\mathrm{SiN}_{\mathrm{x}}$ membrane, Figure 22. A closeup of the model grid of the sensors active area is shown in Figure 29. The grid becomes denser for the parts of the membrane where heaters and thermopiles are located. To reduce the model size and computational time, the silicon frame was omitted and the temperature at the edge of the membrane was set to the frame temperature. For the periodic heat flow the frame is considered as an isothermal heat sink at the slowly changing block temperature.

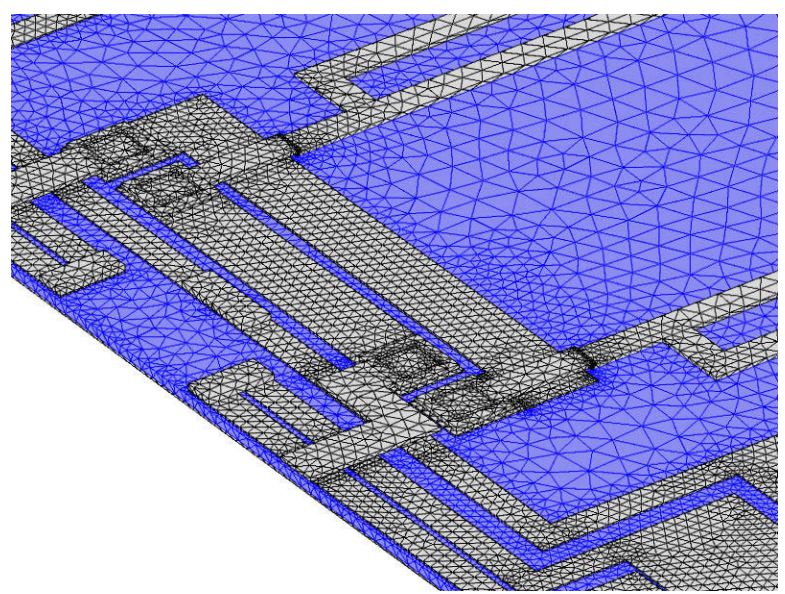

Figure 29: Close-up of the model grid of the sensor's active area. The grid becomes denser where the thermopiles and heaters are located.

The physics that governs the sensor's function is Joule heating causing heat flow into the sample and the membrane, for which the silicon frame acts as an isothermal heat sink. Due to the good vacuum $\left(10^{-7} \mathrm{~Pa}\right)$ and the low temperatures $(\approx 100 \mathrm{~K})$, no other heat conduction paths (convection or radiation) need to be considered [95]. The contribution of radiative heat from the surroundings to the sensors is negligible because the sensor shutter shields the sensors from radiation coming from the room temperature surroundings. The Joule heating at thermal frequency $f_{t h}$ is generated by a current at $f_{t h} / 2$ supplied to the inner heater of the sensor. A thermal wave is generated and propagates laterally towards the inner and the outer part of the membrane. The thermocouple registers the temperature oscillations of the active area relative to the cold junctions placed on the isothermal frame. Therefore the thermopile is measuring the difference between the frame temperature and that of the active area. This difference consists of two parts: (i) the oscillating part and (ii) a constant temperature increase of less than $1 \mathrm{~K}$ due to the DC component of the AC power (DC bias). The oscillating part contains the information of heat 
capacity and thermal conductivity of the membrane and the sample, while the DC part causes an approach toward a steady state depending on the power and only the thermal conductivity of the membrane and the sample. Both parts must be considered in the model and the correct complex temperature amplitude is only available after reaching the steady state for the DC part. The further modeling is restricted to the heat transfer problem and all electrical conversions (current to power and thermopile voltage to temperature) are omitted in the FEM calculations.

In the experiment the power applied to the heater is measured directly. Thus, the applied heat flux $Q$ in $\mathrm{W} \mathrm{m}^{-3}$ is known and is used as an input for the three dimensional model for the heat transfer, see Equation (34).

$$
\rho c_{p} \frac{\partial T}{\partial t}+\nabla \cdot(-\lambda \nabla T)=Q
$$

This equation governs pure conductive heat transfer in a solid, where $\rho$ is density, $c_{p}$ is specific heat capacity in $\mathrm{J} \mathrm{kg}^{-1} \mathrm{~K}^{-1}$ at constant pressure, $\lambda$ is thermal conductivity, $T$ is temperature, $t$ is time, $\nabla \bullet$ stands for divergence and $\nabla$ stands for gradient operators. The temperature dependent material parameters used in the modeling are given in Table I. In the vacuum system there is no convective heat loss and radiation can be neglected too as discussed above. Therefore Equation (34) alone can describe the physics of the system. In our case the model is three dimensional; the temperature distribution spans over lateral and vertical directions. Here heat flux $Q$ is the heat rate generated or absorbed in a given volume. In other words if we integrate the heat flux over the heater volume, where the heat is generated by Joule heating, we will get the power applied to the system.

To further simplify the model symmetry arguments were used. As can be seen in Figure 30 (a) (black line), there is a line of symmetry just between the electrical leaders of the thermopile. Therefore only half of the silicon membrane was modeled and at the line of symmetry, adiabatic conditions were assumed, Figure 30 (b)-(d). 


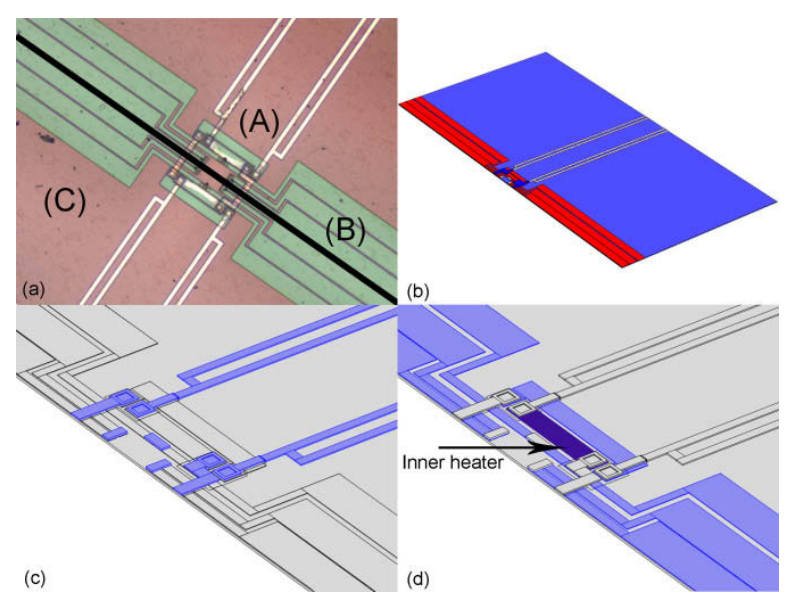

Figure 30: Scheme of the central part of the membrane including the active area (a). (A) indicates the white aluminum electrical leads, (B) the thermocouple stripes and (C) the free standing silicon nitride membrane. The diagonal black line indicates the line of symmetry. The aluminum interconnections, heaters and thermopiles as used in the model are shown in (b) to (d) in more detail. The thermopiles and the heater stripes are made from $300 \mathrm{~nm}$ thick doped poly silicon. They are in green in (a) and in blue in (d). The inner heater, providing the power, is marked as the dark area in (d).

In the experiment and in the model heat is generated in the inner heater. The model assumes that the heater stripes, the dark blue colored part in Figure 30 (d), uniformly distribute heat. The geometrical model of the sensor is then implemented in the COMSOL Multiphysics ${ }^{\circledR}$ FEM package [101]. The software generates 502,473 tetrahedral elements describing the three dimensional structure of the sensor membrane for the FEM analysis (see Figure 29). The material properties used for the FEM as input quantities, besides geometry and heat, are summarized in Table I. The geometrical properties in the model were taken from the data sheet of the modeled sensor [102] and from Ref [103].

Density was not treated as a function of temperature because a constant volume model was considered for the FEM. This way all mechanical stresses were neglected. In the small temperature range of about $20 \mathrm{~K}$ considered here the related error is negligible at this state of the modeling. 
Table I: Material properties of the membrane constituents. Toluene was used as the sample.

\begin{tabular}{lccc}
\hline \hline Material & $\lambda / \mathrm{W} \mathrm{m}^{-1} \mathrm{~K}^{-1}$ & $c_{p} / \mathrm{J} \mathrm{kg}^{-1} \mathrm{~K}^{-1}$ & $\rho / \mathrm{kg} \mathrm{m}^{-3}$ \\
\hline \hline $\mathrm{SiN}_{\mathrm{X}}$ & $\lambda(\mathrm{T})_{\mathrm{SiNx}}{ }^{\mathrm{a}}$ & $c_{p}(\mathrm{~T})_{\mathrm{SiNx}}{ }^{\mathrm{b}}$ & $2843^{\mathrm{c}}$ \\
$\mathrm{SiO}_{2}$ & $\lambda(\mathrm{T})_{\mathrm{SiO} 2}{ }^{\mathrm{d}}$ & $c_{p}(\mathrm{~T})_{\mathrm{SiO} 2}{ }^{\mathrm{e}}$ & $2200^{\mathrm{c}}$ \\
Aluminum & $\lambda(\mathrm{T})_{\mathrm{Al}}{ }^{\mathrm{f}}$ & $c_{p}(\mathrm{~T})_{\mathrm{Al}}{ }^{\mathrm{f}}$ & $2728^{\mathrm{g}}$ \\
Poly silicon & $30^{\mathrm{c}}$ & $770^{\mathrm{c}}$ & $2300^{\mathrm{c}}$ \\
Toluene & $\lambda(\mathrm{T})_{\text {toluene }}{ }^{\mathrm{h}}$ & $c_{p}(\mathrm{~T})_{\text {toluene }}{ }^{\mathrm{i}}$ & $1025.4^{\mathrm{j}}$ \\
\hline \hline
\end{tabular}

${ }^{a}$ From reference [104], Figure 9b

${ }^{\mathrm{b}}$ From reference [105] with the temperature dependence from reference [106], Figure 3.

${ }^{c}$ From reference [107], Table A2

${ }^{\mathrm{d}}$ From reference [108], Table 14, Quartz (Cleared Fused)

${ }^{\mathrm{e}}$ From reference [109], Table 12, amorphous $\mathrm{SiO} 2$

${ }^{\mathrm{f}}$ From reference [101], built in function.

${ }^{\mathrm{g}}$ From reference [101] at $120 \mathrm{~K}$

${ }^{\mathrm{h}}$ From reference [110], Table 2.

${ }^{\mathrm{i}}$ From reference [111], Table 1.

${ }^{\mathrm{j}}$ From reference [112], Table 4, at $0.1 \mathrm{MPa}$ at $120 \mathrm{~K}$.

First, the static behavior of a single sensor was modeled. For the calculation of the static behavior the steady state solution of (34) can be used:

$$
\nabla \cdot(-\lambda \nabla T)=Q
$$

The only material property that is included in the steady state calculation is thermal conductivity, $\lambda$. Therefore the model is first compared with the experiment for the static case. A stepwise increasing DC power was applied to the heater and the resulting temperature rise was calculated and compared with the corresponding experimental data. In Figure 31 one sees good agreement between the model and the experiment. 


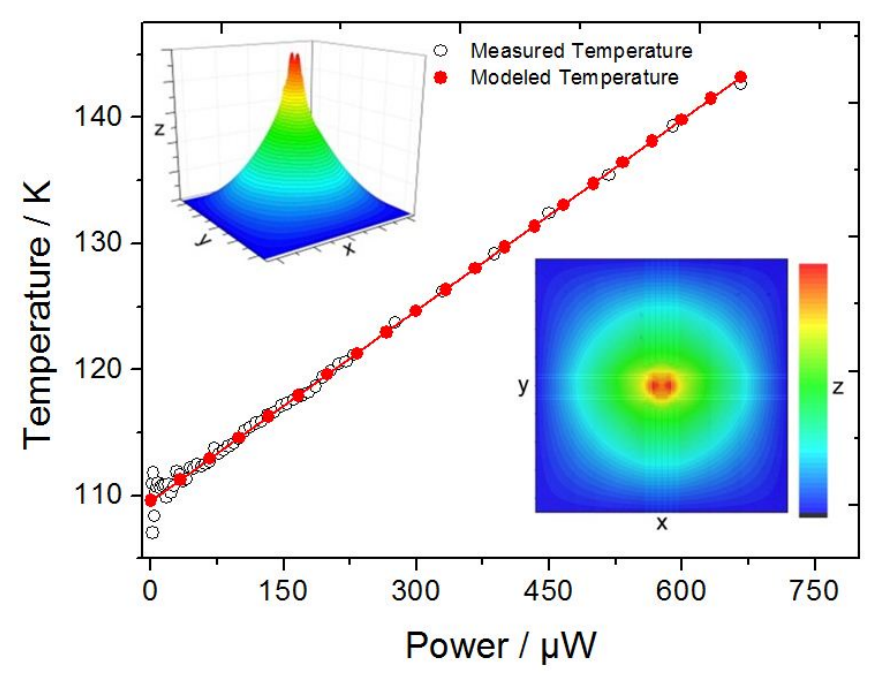

Figure 31: Steady state comparison between the experimental data (open circles) and the model data (dots). The power was applied to the inner heater and in the experiment the temperature was recorded by the heater resistivity. The silicon frame temperature was $110 \mathrm{~K}$. The insets show, for illustration only, the normalized temperature distributions from the FEM calculations for a DC-heated chip sensor in vacuum. The scaling is as follows: $\mathrm{X}$ and $\mathrm{Y}$ directions span 0 to $900 \mu \mathrm{m}$ and $\mathrm{Z}$ is normalized temperature between 0 and 1 .

Next, the dynamic behavior of a single sensor was investigated and compared to the experimental data. The frequency range was $0.2 \mathrm{~Hz}$ to $1 \mathrm{kHz}$. There is no need to expand the frequency range further, because the main and most important features of the dynamic behavior are covered by this range. Because the experimental data acquired from the lock-in amplifier is given as RMS values, it has to be multiplied by $\sqrt{2}$ for comparison with the simulated amplitude data. The data for a single sensor are shown in Figure 32.

The dynamic simulation in COMSOL was performed using the time dependent solver and is described next. A power of thermal frequency $f_{t h}$ and amplitude $4.57 \mu \mathrm{W}$ was applied to the inner heater stripe of the model, see Figure 30 (d). The power that was used in the experiment is twice that $(9.14 \mu \mathrm{W})$ and applied to both inner heater stripes of the sensor. The model needs half of the power used in the experiment since the geometrical model covers only half of the membrane to save computational time. Due to the DC bias the sensor has a transient phase of about $40 \mathrm{~ms}$ at the beginning. When the membrane DC temperature rise equilibrates, the amplitude of the temperature oscillation equilibrates. 

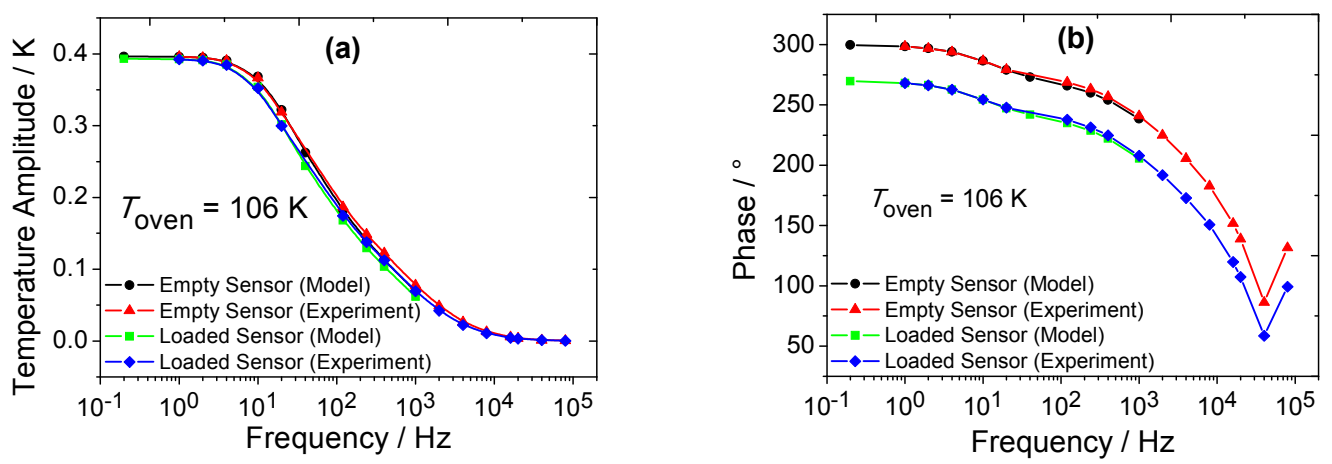

Figure 32: Comparison of simulated (circle, square) and experimental (triangle, diamond) data points for an empty sensor and a sensor loaded with a $390 \mathrm{~nm}$ toluene sample. Temperature amplitude is shown in (a) and the phase angle between temperature and power in (b).

After the stabilization, three periods of the oscillation were used for amplitude and phase determination. This was repeated for three frequencies per order of magnitude. The obtained data points for the empty sensor are compared to the experimental points in Figure 32. After successful modeling of the empty sensor, a layer of toluene was added to the model and the modeled data were compared to the corresponding experimental data. Because sample thickness for the experimental data is not known it cannot be used as an input for the simulation. Therefore the film thickness was varied in the simulation at $20 \mathrm{~Hz}$ (which is the thermal frequency used in the measurements) until the obtained temperature amplitude at $106 \mathrm{~K}$ oven temperature equaled the measured one. From this iterative procedure a toluene film thickness of $390 \mathrm{~nm}$ was determined for the films commonly deposited in the experiments.

The modeled and the measured curves are in good agreement. The maximum deviation in the differential temperature amplitude is approx. $2 \mathrm{mK}$ and in the phase approx. $5^{\circ}$. In the low frequency region, the simulated values are slightly lower while at high frequencies, starting from $120 \mathrm{~Hz}$, the calculated values are lower than the experimental curve, Figure 32 (a). In the phase, Figure 32 (b), only the high frequency data deviate a bit from the measured values.

From Figure 32 (a) the difference between the empty and the sample-loaded sensor is hard to notice because the $390 \mathrm{~nm}$ thick sample does not change the signal significantly. In the experiment, we use a differential setup because of much higher sensitivity. The difference between the calculated and measured signals for the loaded and empty sensor was performed in the complex plane. Figure 33 shows that the fit of the differential experimental data produced by the model is excellent. 

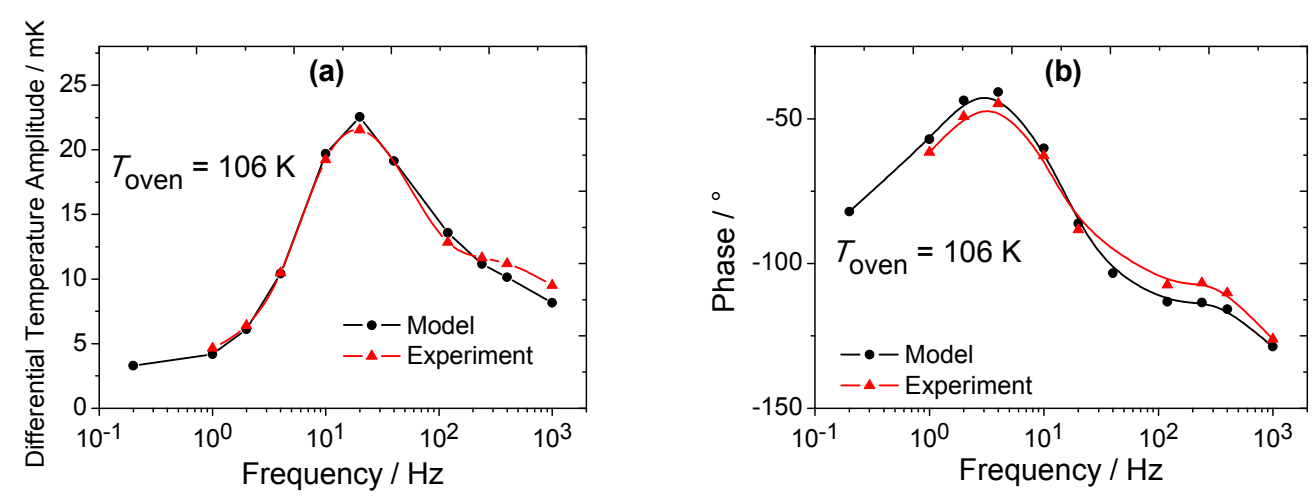

Figure 33: Differential temperature amplitude (a) and phase angle between temperature and power for the differential signal (b) for a $390 \mathrm{~nm}$ toluene film. Experimental differential data are shown as circles and the modeled data are shown as triangles.

Next, the model was checked not only at one fixed temperature but also over the temperature range of interest. The empty sensor was measured between $107 \mathrm{~K}$ and $126 \mathrm{~K}$ and the data were compared with the model. Then the toluene layer with thickness $390 \mathrm{~nm}$ was added to the model and the resulting temperature amplitudes were determined for different temperatures outside the glass transition region. Points at temperatures in the glassy and the liquid state were chosen. Because the difference between the empty sensor and the sample loaded sensor is small and hard to observe in Figure 32, again the differential signal was compared with the experimental data to allow the quality of the model data to be more easily demonstrated. As these are complex numbers the difference was calculated in the complex plane. As seen in Figure 34 the agreement in the glassy and the liquid state is good for the differential amplitude and a constant offset of 3 degrees is observed for the phase. After correction for this offset the agreement is also good. The offset in the phase is caused by an additional time constant in the electronics.

It must be mentioned here that as an input for the model the temperature dependent specific heat capacity of toluene from adiabatic calorimetry was used [111]. Due to the AC nature of the experiment, the frequency dependent dynamic glass transition temperature is measured rather than the standard glass transition obtained from the adiabatic data. Therefore the simulation is not able to model either the step change of the signal at the glass transition or the measured peak in the phase angle. 

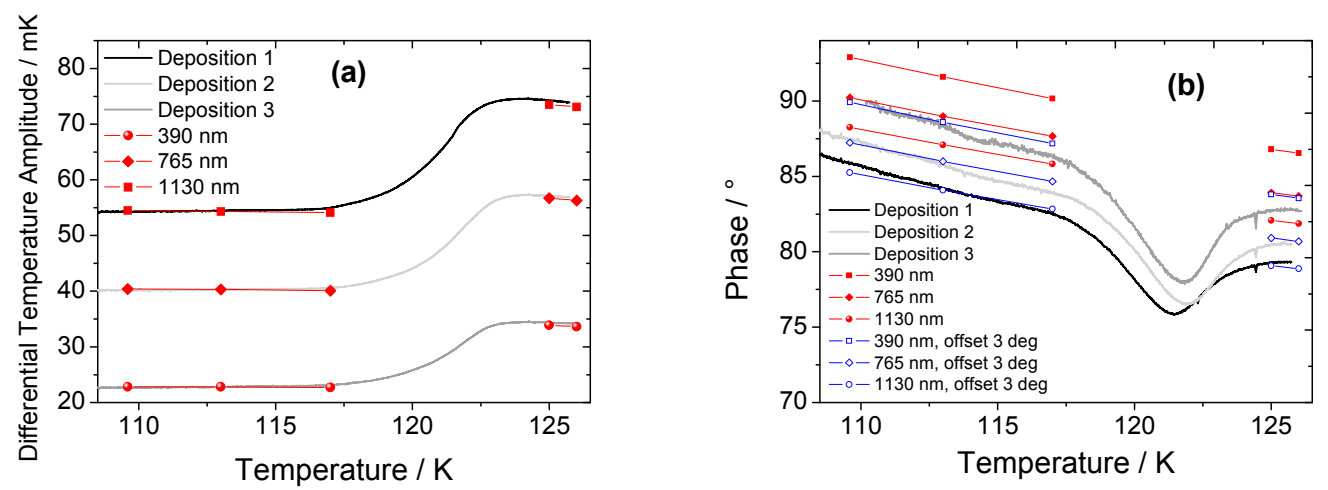

Figure 34: Experimental and modeled data for three films of slowly cooled toluene glasses of different thickness; the differential amplitude (a) and the phase angle between power and temperature (b). Experimental data are black, light gray and gray curves and the corresponding modeled data are squares, diamonds and dots, respectively. In (b) the phase data are shifted by $3^{\circ}$ (open symbols) to compensate for additional time constants from the electronics.

Figure 34 proves that the model is capable of reproducing the measured signal for a sample with only slightly changing heat capacity. The model was also checked for toluene films of different thickness to determine the accuracy for samples that have larger changes in heat capacity. The nonlinear relationship of the measured differential temperature amplitude as a function of deposition time for a deposition at constant rate can be seen in Figure 35. The inset shows the QCM signal as a relative measure of sample thickness over deposition time, verifying the constant deposition rate. To construct the top axis of Figure 35, the fixed point at $390 \mathrm{~nm}$, determined above, and the constant deposition rate were used to convert deposition time to sample thickness. The sample thickness was then used as an input parameter for the modeling and again modeled and experimental amplitude data are in good agreement. 


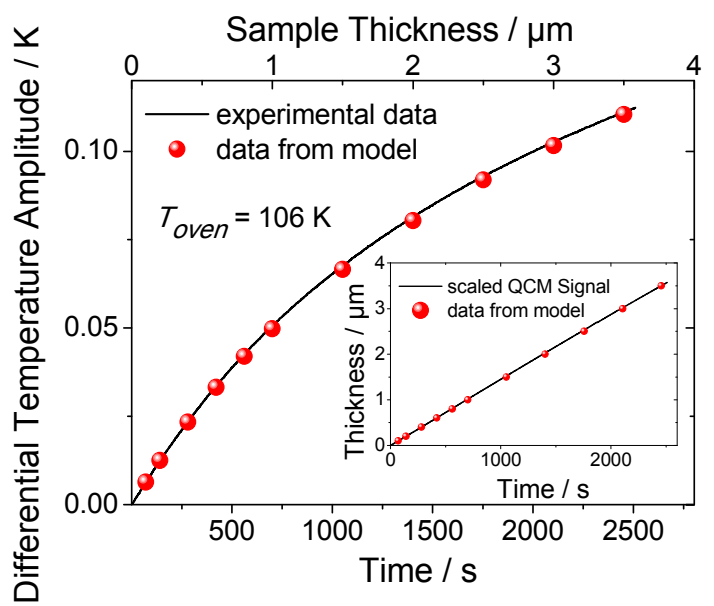

Figure 35: The black curve corresponds to the measured differential temperature amplitude during deposition of toluene and the red points show the modeled data with different thicknesses. The modeling and experiment were done at $106 \mathrm{~K}$ and a thermal frequency of $20 \mathrm{~Hz}$. The deposition rate was held constant at about $1.5 \mathrm{~nm} \mathrm{~s}^{-1}$. The inset shows the temporal evolution of the sample thickness.

Summarizing, the finite element method provides a description of the static as well as the dynamic behavior of single sensors and the differential signals for empty and sample loaded sensors. The model is capable of reproducing the measured signal for a changing sample heat capacity. It also allows the thickness of the deposited films to be determined despite the nonlinear dependence of the complex temperature amplitude on film thickness. The absolute film thickness determined by comparison with the model has an uncertainty of $\pm 20 \%$ because of uncertainties in the input parameters, e.g. conversion of thermopile voltage to temperature, material properties, etc. The measurement precision is much higher and the difference in thickness between two films can be determined with much higher accuracy (few nm). Unfortunately, at this time there are no independently determined values of sample thickness available and therefore the total uncertainty of sample thickness is only roughly estimated as $\pm 20 \%$. In the future the FEM results may be checked by ellipsometric measurements in the deposition chamber.

The absolute sample heat capacity determination remains still unsolved. Even though the FEM is able to evaluate the film thickness of the sample, the fraction of the sample contributing to the differential temperature amplitude is unknown and thus a reliable heat capacity calculation is not possible. 


\subsubsection{DC signals}

The DC voltage measures the DC overheating of the membrane and thus can be used for measuring endothermic or exothermic effects like crystallization. This quantity is the preamplified differential thermopile voltage as measured by the lock-in amplifier. The voltage can be tapped from the signal monitor (SIGMON) output of the lock-in amplifier. The amplification corresponds to what is set at the lock-in amplifier by the operator. The signal from the SIGMON output is superimposed by the $2 \omega$ oscillation. This oscillation is erased from the signal using a passive low pass filter using an $\mathrm{RC}$ circuit. The resulting DC voltage which can be expressed as the overheating of the membrane is measured by an Agilent digital multimeter.

The characteristic feature in the DC voltage coming from the glass transition is not observed since the effect is very small and thus hidden by noise. The glass transition is a kinetic event and thus changes in the DC voltage at the glass transition originate from changes in the thermal resistance of the sample if the frozen-in structure melts. As a consequence, the overheating of the membrane of the sample sensor changes. However, this change is small and thus not seen in the DC voltage. Crystallization and melting produce well pronounced peaks as can be seen in Figure 36 where the DC voltage and the differential temperature amplitude of a temperature ramping of a toluene sample are shown over the heater resistance.

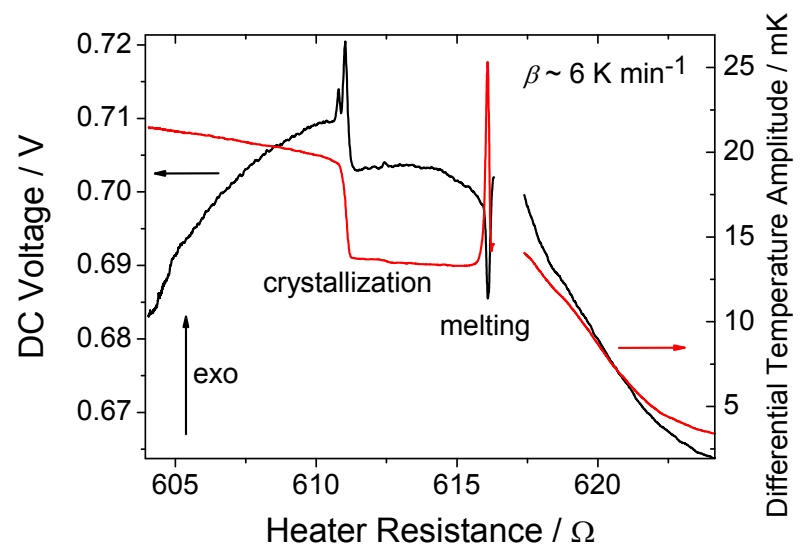

Figure 36: DC voltage (black) and differential temperature amplitude (red) over heater resistance during heating of a thin toluene sample starting above the glass transition. The heating rate is set to maximum. The peak at $611 \Omega$ can be associated with crystallization whereas the peak at $616 \Omega$ is the melting of toluene. The decreasing trend at higher heater resistance (higher temperature) shows evaporation of the sample. 
Crystallization occurred at about $611 \Omega$ and the differential temperature amplitude drops from the liquid heat capacity to the crystal heat capacity. At this point the DC voltage shows an exothermic peak. The whole membrane of the sample sensor is heated up by the produced heat during crystallization resulting in a higher difference between the membrane temperatures of reference and sample sensor. This can be seen as a crystallization peak in Figure 36.

At about $616 \Omega$ the crystalline toluene melts which is indicated by an endothermic peak in the DC voltage. The differential temperature amplitude shows the melting peak too.

However, the focus of this work is not crystallization and melting, but rather than the glass transition. Even though a detailed discussion of the crystallization and melting of toluene and ethylbenzene is possible it is not further elaborated on here.

\subsection{Temperature calibration}

\subsubsection{Temperature measurement}

A Pt100 resistive thermometer is used for measurement of the temperature of the copper block. Figure 37 shows the differential temperature amplitude (solid line) and the phase (dashed line) of the subsequent $1^{\text {st }}$ cooling and $2^{\text {nd }}$ heating of the glass transition of a toluene sample plotted over the Pt100 temperature. The characteristics of the complex heat capacity for the glass transition can clearly be seen: (i) the measured phase difference between the temperature oscillation as measured with the thermopiles and the applied power shows a minimum at the glass transition due to the relaxation process and (ii) the differential temperature amplitude exhibits a step-like change caused by the time constant originating from the changing heat capacity and the thermal resistance between the sample and the heater [113].

A system with a sample with ideal thermal coupling to the bath would show no temperature hysteresis. As can be seen from Figure 37 a heating/cooling rate of $0.67 \mathrm{~K} \mathrm{~min}^{-1}$ gives a thermal hysteresis between Pt100 temperature connected to the bath and differential temperature amplitude with a temperature lag of $0.8 \mathrm{~K}$ comparing heating and cooling due to non-ideal thermal coupling. This means the membrane temperature of the sensor lags behind the Pt100 temperature. Moreover the mean dynamic glass transition temperature of $131 \mathrm{~K}$ as determined from Figure 37 is $8.5 \mathrm{~K}$ above the expected dynamic glass transition temperature of $122.66 \mathrm{~K}$. From the experience with the device it was found that this difference can change from one temperature cycle to a subsequent one and thus cannot be used for correction of the Pt100 temperature. The temperature determination requires higher accuracy because the strong 
temperature dependence of the structural relaxation time for fragile glass formers like toluene necessitates knowing the temperature precisely.

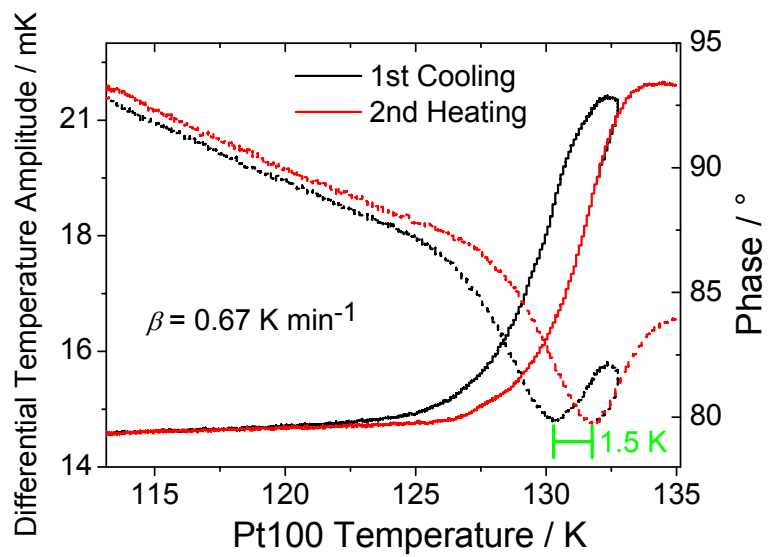

Figure 37: Measured differential temperature amplitude (solid lines) and phase (dashed lines) for heating (red) and cooling (black) of toluene over Pt100 temperature. Since the Pt100 and the thermopiles of the sensor are not located at the same position and thermal connection to the oven is most probably not equal for both the heating and cooling curves show a hysteresis of about $1.5 \mathrm{~K}$ at a heating rate of $0.67 \mathrm{~K} \mathrm{~min}^{-1}$.

The wrong absolute Pt100 temperature may have its explanation in bad thermal coupling of the wiring. Even if the wires are encapsulated by a network of steel fibers and clamped to the copper block, the connections at the electrical feedthrough introduce a temperature gradient leading to the wrong absolute values of the Pt100 resistance.

\subsubsection{Heater resistance}

In a vacuum environment at low temperatures, when radiation between neighboring parts is not effective for heat transfer, temperature calibration is a challenging task because heat transfer is limited to the heat conduction of solid parts in intimate contact. Consequently, large temperature gradients between different parts, e.g. the temperature sensor and the sample, may exist. For example, see Figure 37 for the temperature measurement using the Pt100 employed for the temperature control. A thermal lag of $0.8 \mathrm{~K}$ between the Pt100 and the sample is detected which is not acceptable because of the strong temperature dependence of the structural relaxation of the investigated substances, see Figure 42 and Figure 44. Therefore, it is preferential to measure the sample temperature as close as possible to the sample and to ensure good thermal contact between the sample and the thermometer. In the case of a thin film calorimeter sensor, 
measuring the temperature of the membrane underneath the sample is recommended. For vapordeposited samples, the thermal contact between the sample and the membrane, created during the deposition, is very good.

Two thermometers are available underneath the sample. One is the thermopile, which measures the temperature difference between the sensor housing and the working area of the membrane. Using the thermopile for absolute sample temperature determination requires knowing the housing temperature and therefore the Pt100 is still needed for the temperature measurement. Consequently, the thermopile is not very useful for measuring the absolute sample temperature and is used here only for detecting the temperature oscillation. The other available thermometer is the heater resistance. The temperature dependence of the heater resistance and the high reproducibility enables the use of $R_{h}$ as a resistive thermometer (RTD) for absolute temperature measurement very close to the sample without the need for any other temperature measurement. In a 4-wire setup, the temperature dependent heater resistance can be determined without additional contributions coming from connections. It is calculated from the voltage drop $U_{i}$ over a known pre-resistance and the voltage drop $U_{h}$ over the heater resistance of the sample sensor (Figure 23). This gives the absolute temperature used for the $\mathrm{AC}$ calorimetric experiment and thus the temperature of the sample itself with an error of $\pm 1 \mathrm{~K}$ as determined from the standard deviation of the heater resistance measurement.

Measuring the temperature of the sample directly at the sample position eliminates the influence of the thermal lag between the copper block and the working area on the sensor membrane. The remaining thermal lag between the differential temperature amplitude and the heater resistance is characterized by the internal time constant of the sensor, which is on the order of a few milliseconds. An example of using the heater resistance for temperature measurement is given in Figure 38. However, due to small differences, each sample sensor needs separate temperature calibration of the heater resistance. 


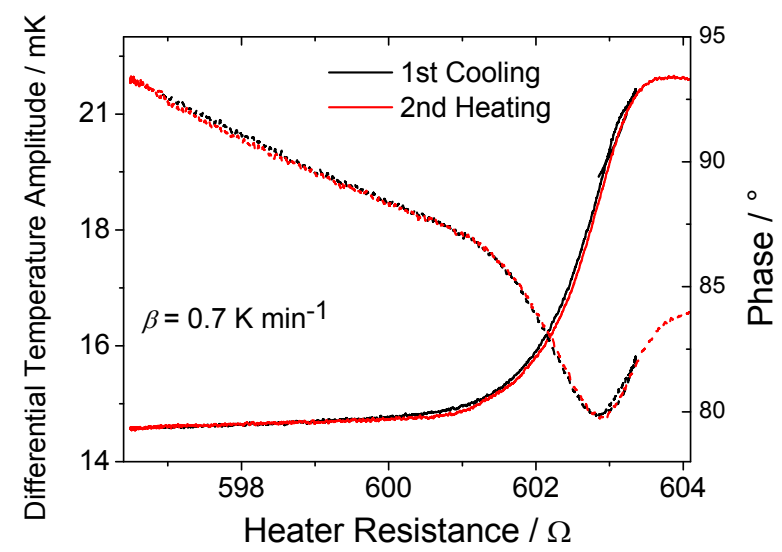

Figure 38: Differential temperature amplitude (solid lines) and phase (dashed lines) over heater resistance for a thin toluene sample. As can be seen the hysteresis between heating and cooling vanished.

The sensors were not heated above $350 \mathrm{~K}$ because the electrical resistance of the onmembrane heater of the calorimetric sensor changes irreversibly at elevated temperatures. Since it is used as a thermometer to compensate for all thermal lags between the copper block and the central, heated part of the sensor membrane, a shift of the heater resistance causes the calibration function to become invalid. This implies that the chamber with the equipped calorimeter cell cannot be baked at temperatures higher than $350 \mathrm{~K}$. This ensures the cell including the sensors is the hottest place in the chamber and possible dirt in the chamber will accumulate at the cooler chamber walls if not transported away by the vacuum pumps.

However, reliable membrane temperature determination from the heater resistance measurement is possible only up to about $180 \mathrm{~K}$ with the current calibration (see section 3.3.3). In this respect the range of reliable temperature determination is between $92 \mathrm{~K}$ and $180 \mathrm{~K}$.

\subsubsection{Temperature calibration of the heater resistance}

Calibration is challenging since it has to be done in-situ without corrupting the sensors permanently. Once a sample sensor is installed the calibration, some test-measurements and at least one series of measurements should follow. Thus calibration needs volatile materials having transitions of any kind in the temperature range of interest. Moreover these substances should be easy to deposit without sticking to the chamber walls and rotor blades of the turbo pump that are near room temperature. This makes it necessary to use substances with a high vapor pressure at room temperature. 
One option is to use cyclopentane, which is volatile at room temperature and ambient pressure, and has three transitions spanning a range of $56 \mathrm{~K}$ from $122 \mathrm{~K}$ to $178 \mathrm{~K}$; unfortunately cyclopentane provides no calibration points at temperatures below $122 \mathrm{~K}$. Nevertheless the broad temperature range spanned by the cyclopentane transitions allows reliable extrapolation to lower temperatures.

Another option to determine the calibration function is to measure the frequency dependent glass transition temperature (in terms of heater resistance) of toluene and ethylbenzene and combine the data with the temperatures calculated from the corresponding VFT equations. For these compounds precise measurements of the molecular dynamics, i.e. the VFT equation, can be found in the literature $[114,115]$. However, the possible frequency variation with the current device spans only 3 orders of magnitude, which only gives a change in the dynamic glass transition of less than $10 \mathrm{~K}$ since these two compounds are very fragile. This range is too small to allow a reliable extrapolation of the calibration function to temperatures down to the lowest deposition temperatures. However, the frequency dependent glass transition temperatures of toluene as well as ethylbenzene are used for re-checking the calibration. Furthermore the frequency dependent glass transition temperature of ethylcyclohexane [116] is used to check the reliability of extrapolation of the resulting calibration function.

\subsubsection{Calibration with cyclopentane}

Cyclopentane is an alicyclic hydrocarbon and its chemical formula is $\mathrm{C}_{5} \mathrm{H}_{10}$. This means it consists of five carbon atoms each bonded with two hydrogen atoms above and below the plane (see Figure 39).

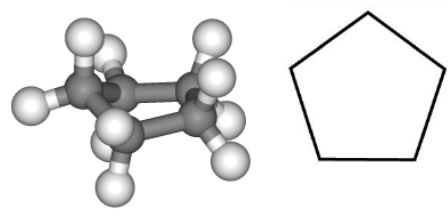

Figure 39: $3 \mathrm{D}$ and 2D drawing of cyclopentane [from http://en.wikipedia.org/wiki/Cyclopentane].

At room temperature cyclopentane is in the liquid phase and slightly volatile which makes it a good candidate for physical vapor deposition. The most important reason for using cyclopentane as a calibrant are the three phase transitions it exhibits. These transitions are the solid phase III-to-plastic phase II, the plastic phase II-to-plastic phase I and the plastic phase I-toliquid transitions [117]. Jakobi et al. [118] measured the transition temperatures with a high precision adiabatic calorimeter to be $122.23 \mathrm{~K}, 138.35 \mathrm{~K}$ and $178.59 \mathrm{~K}$, respectively. 
The dynamic glass transitions of toluene and ethylbenzene at $f_{t h}=20 \mathrm{~Hz}$ are in close vicinity to the solid-to-plastic crystal II transition of cyclopentane. Thus the error coming from the interpolation between the calibration points is very small regarding the glass transition temperature determination. Nevertheless, the resulting calibration function has to be extrapolated to determine the deposition temperatures. Due to the relatively large temperature range that is spanned by the three transitions the extrapolation error down to about $95 \mathrm{~K}$ is assumed to be sufficiently small (see section 3.3.3.4).

Cyclopentane is vapor-deposited at $105 \mathrm{~K}$ to yield a film of $\sim 2 \mu \mathrm{m}$. Only the solid phase III-to-plastic phase II transition can be measured in vacuum since sublimation occurs at higher temperature. Backfilling the chamber with nitrogen gas up to 300 mbar avoids sublimation but allows heat loss through the gas, in addition to the heat loss through the membrane, which may influence the temperature distribution in the membrane [100]. To check the influence of the gas, the temperatures of the solid phase III-to-plastic crystal II transition measured in vacuum and in nitrogen gas were compared. All experimental parameters except the pressure were equal. The heating rate was set to $0.67 \mathrm{~K} \mathrm{~min}^{-1}$ to ensure controlled heating; this is different than the heating rate used in the adiabatic experiments as done by Jakobi et al. $\left(2 \mathrm{~K} \mathrm{~min}^{-1}\right)$ [118]. It is not expected that this small difference in heating rate or the small temperature oscillations used for $\mathrm{AC}$ calorimetry affect the transition temperatures.

The difference between vacuum and nitrogen measurements of the solid phase III-toplastic crystal II-transition is about $0.4 \mathrm{~K}$ and thus within the uncertainty of the temperature determination of $\pm 1 \mathrm{~K}$ (see section 3.3.3.4). Thus we conclude temperature calibration at ambient pressure using cyclopentane can be applied to measurements in vacuum.

The calibration points are determined from the phase of the complex thermopile signal (see Figure 40) since the sharpness of the phase peaks allows a more precise determination of the calibration points and thus ensures high consistency with the measurements from adiabatic calorimetry [118]. The calibration points together with a second order polynomial fit function are shown in Figure 45 below. 


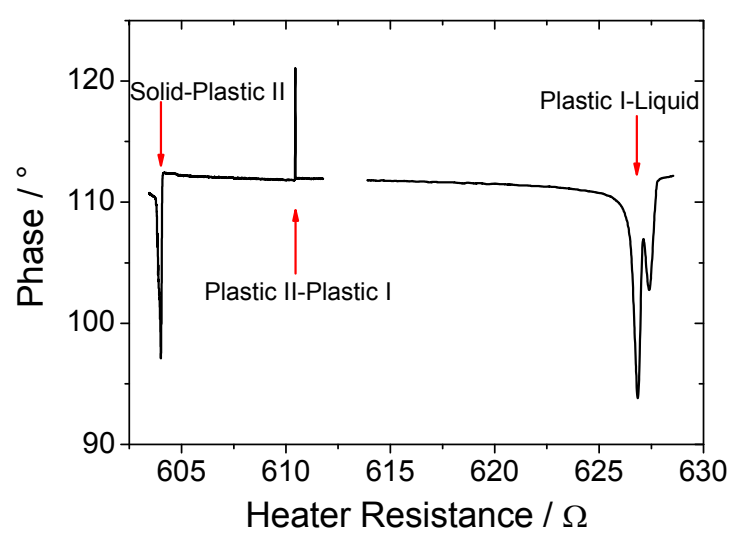

Figure 40: Phase angle of the complex reversing heat capacity of cyclopentane over heater resistance. The measurement was done at 300 mbar of nitrogen gas and with a heating rate of $0.67 \mathrm{~K} \mathrm{~min}^{-1}$.

The calibration with cyclopentane is re-checked with the frequency dependent dynamic glass transition temperatures of toluene and ethylbenzene.

\subsubsection{Toluene}

Toluene is a mono-substituted benzene derivate which means that a hydrogen atom from the benzene molecule is replaced by a $\mathrm{CH}_{3}$ methyl group. In this respect it has a very simple structure (Figure 41). It belongs to the aromatic hydrocarbons and is mainly used as a solvent for paints, paint thinners and silicon sealants, moreover lacquers, leather tanners and disinfectants. It is also used as a raw material for trinitrotoluene (TNT).

Toluene can cause health effects such as confusion, memory loss, hearing and color vision loss. Thus it should be handled with due care. In the present work toluene vapor was exposed to the experimenter only during replacing of the sample reservoir. The required amount of toluene for each experiment is very small (about $0.1 \mathrm{ml}$ ) and it is pumped out by the mounted pumps slowly into an exhaust. Thus special care is only needed during refilling of the reservoir. 


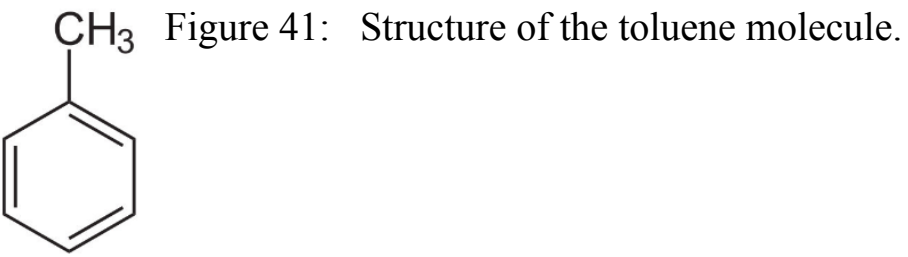

Among other properties of toluene, the structural relaxation times as well as the specific heat capacity are of high interest in this work.

The first calorimetric measurements on toluene at cryogenic temperatures were made by Kelley in 1929 [119]. Unfortunately liquid toluene was measured only down to 184 K. Later Scott and Guthrie [120] did more precise measurements of the heat capacity of liquid toluene but again only down to $178 \mathrm{~K}$. This is because toluene tends to crystallize easily. To bypass this problem Yamamuro [111] et al. doped the toluene sample with about $10 \%$ of benzene which suppressed crystallization. Since it is assumed the heat capacities are additive, the doping effects can be subtracted. They determined the heat capacity by adiabatic calorimetry. Their results are used in the present work.

Measurements of the $\tau_{\alpha}$ structural relaxation time were done with different methods. Hinze et al. [121] used ${ }^{2} \mathrm{H}$ nuclear magnetic resonance (NMR) to determine the relaxation time in the temperature range relevant to this work and got $\tau_{0}=6.34 \times 10^{-15} \mathrm{~s}, B=791 \mathrm{~K}$, and $T_{0}=95 \mathrm{~K}$ for the VFT parameters. Döß et al. [122] used dielectric spectroscopy (DR) for the measurement of the relaxation time of toluene which was doped with thin-walled capillaries with outer diameters of $300 \mu \mathrm{m}$ to prevent crystallization. The VFT parameters were found to be $\tau_{0}=1.5 \times 10^{-15} \mathrm{~s}, B=729.7 \mathrm{~K}$, and $T_{0}=96.5 \mathrm{~K}$. Furthermore they calculate the fragility index $m$ to be 105. Kudlik et al. [123] used dielectric susceptibility (DS) measurements to examine both the structural and the slow secondary relaxation process of toluene. The fragility index $m$ is reported to be 122. Floudas et al. $[124,125]$ reported the VFT as determined from depolarized Rayleigh scattering (DRS) with the parameters $\tau_{0}=2.8 \times 10^{-13} \mathrm{~s}, B=251 \mathrm{~K}$, and $T_{0}=96 \mathrm{~K}$. Hatase et al. [115] lumped the reported structural relaxation times data as determined by Kudlik, Hinze, Rössler [126] and Yamamuro [111] and found the parameters for the VFT as $\tau_{0}=6.3 \times 10^{-13} \mathrm{~s}, B=434 \mathrm{~K}$, and $T_{0}=104 \mathrm{~K}$.

A reliable VFT equation is required for later data analysis. Figure 42 displays the different VFT equations to determine which of the VFTs is the most reliable one.

Since the VFT of Hatase and the DS data of Kudlik as well as the adiabatic data of Morineau almost coincide and the $T_{g}$ of the Hatase VFT at $\tau_{\alpha}=100 \mathrm{~s}$ is $117.3 \mathrm{~K}$, which is widely 
supported in the literature, the VFT determined by Hatase et al. is considered to be the most reliable in the temperature range of interest in this work. This does not imply the other VFTs are wrong, but due to limited data in the temperature range close to the glass transition, some of the VFT fits required extrapolation which may not be reliable. Later on the VFT of Hatase et al. is consulted for calculations of the frequency dependent glass transition of toluene.

For our work we use toluene that is purchased from Fluka ${ }^{\mathrm{TM}}$ and used without further purification. The chemical purity is better than $99.8 \%$.

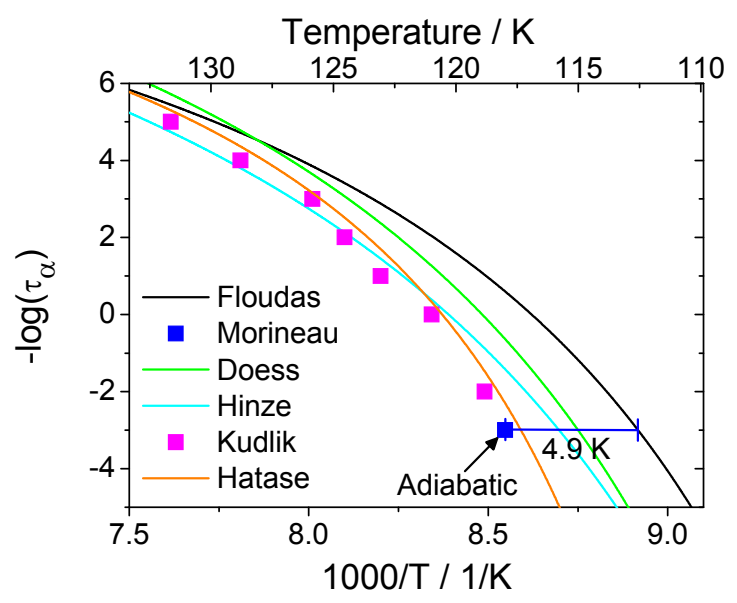

Figure 42: Plot of VFTs and data of the structural relaxation time of toluene found in the literature. The black line is determined from DRS measurements [125], the green line by DR [122], the light blue line by NMR measurements [121], and the orange line is determined by lumping several structural relaxation time measurements together $([111,121,123,126])$. The magenta squares represents data determined by DS [123] whereas the single blue square is taken from adiabatic measurement [127]. There is a huge deviation between the different datasets. At the glass transition the difference between the adiabatic measurement by Morineau et al. and the NMR measurement of Floudas et al. is found to be about $5 \mathrm{~K}$. This deviation may be due to the fact of rare or no data in the temperature region below $120 \mathrm{~K}$ and consequential extrapolation errors.

\subsubsection{Ethylbenzene}

Ethylbenzene is another derivate of the benzene group (see Figure 43). It is industrially of high importance since it is an intermediate in the styrene production from which common plastic materials are produced. Like toluene it can be found as a solvent in many paints. It also can cause health effects like the ones mentioned in section 3.3.3.2. 


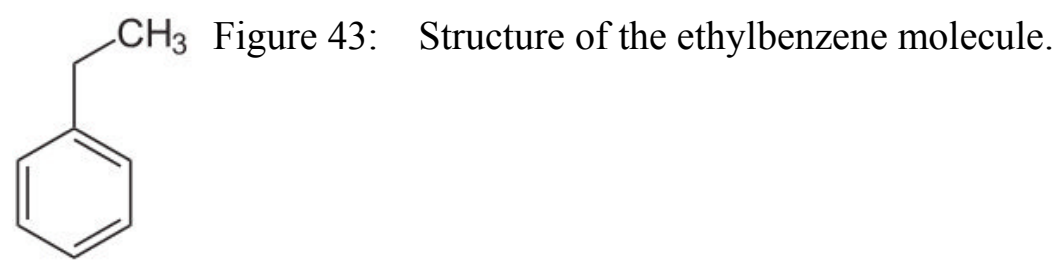

Again heat capacity and structural relaxation time of ethylbenzene are of interest for this work. The heat capacity data are taken from the work of Yamamuro et al. [111] presenting the first calorimetric data on crystalline and pure liquid ethylbenzene.

The literature provides only poor information of the structural relaxation of ethylbenzene. Cutroni et al. [128] and Chen et al. [129] provide VFTs for ethylbenzene determined by acoustic attenuation measurements and dielectric relaxation, respectively.

The VFT equation derived from the ultrasonic spectroscopy measurements by Cutroni et al. is dependent on the acoustic attenuation fit including the Kohlrausch-Williams-Watt or stretching exponent $\beta_{K W W}$, which can be temperature dependent. The sum of two relaxation functions, an Arrhenius-like and a VFT-like function, was found to be the best fit for the acoustic attenuation. A constant stretching parameter yields $\tau_{0}=8.6 \times 10^{-15} \mathrm{~s}, B=1380 \mathrm{~K}$ and $T_{0}=69.4 \mathrm{~K}$ for the parameters of the VFT equation. A temperature dependent stretching parameter yields $\tau_{0}=4.1 \times 10^{-14} \mathrm{~s}, B=883 \mathrm{~K}$ and $T_{0}=95.4 \mathrm{~K}$. The joint Arrhenius-VFT fit, again with a temperature dependent stretching parameter, gives $\tau_{0}=1.2 \times 10^{-15} \mathrm{~s}, B=1040 \mathrm{~K}$ and $T_{0}=98.1 \mathrm{~K}$.

The recently published data of Chen et al. give the VFT parameters for the low temperature range since at higher temperatures the temperature dependence of the dynamics of ethylbenzene does not follow a single VFT law, as is the case for other molecular glass formers. Thus the low temperature VFT parameters are $\tau_{0}=9.12 \times 10^{-16} \mathrm{~s}, B=793.06 \mathrm{~K}$ and $T_{0}=95.45 \mathrm{~K}$.

Figure 44 displays the mentioned VFT functions as determined by Cutroni et al. and Chen et al. The deviation between the curves in the activation diagram is remarkable. As stated in the literature (e.g. [111]) the adiabatic glass transition temperature is about $115 \mathrm{~K}$ which is consistent with the data of Chen et al. and thus it is decided to use the VFT as given by Chen et al. 


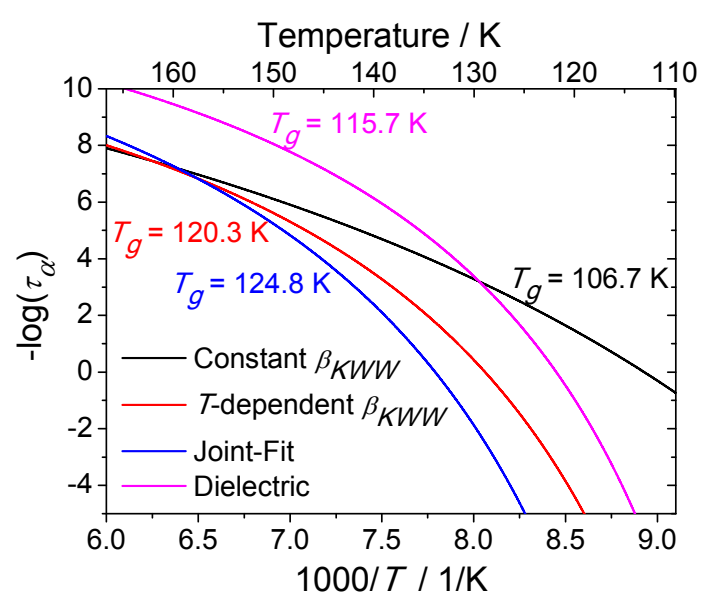

Figure 44: Plot of the VTF functions for ethylbenzene found in the literature. Except for the magenta curve which is taken from [129] the curves are taken from [128]. The glass transition temperatures represent the temperatures at $\tau_{\alpha}=100 \mathrm{~s}$ or $-\log \left(\tau_{\alpha}\right)=-2$. The variation in $T_{g}$ between the VFTs from [128] is about $19 \mathrm{~K}$.

For our measurements we use ethylbenzene that is purchased from Acros Organics and used without further purification. The chemical purity is better than $99.8 \%$.

\subsubsection{Secondary calibration with the dynamic glass transition of toluene and ethylbenzene}

As a secondary check of the temperature calibration (see section 3.3.3.1), the frequency dependence of the dynamic glass transition temperature of toluene and ethylbenzene was measured. An approximately $400 \mathrm{~nm}$ film was deposited onto the chip sensor at $105 \mathrm{~K}$ and then scanned slightly above its glass transition to erase all memory regarding the deposition process before cooling the sample down to about $110 \mathrm{~K}$ with $0.67 \mathrm{~K} \mathrm{~min}^{-1}$. The maximum temperature was chosen such that crystallization and sublimation of the sample was avoided. After the first scan, the sample is an ordinary liquid-cooled glass. The subsequent scans were done with different thermal frequencies spanning 3 orders of magnitude. For every measured frequency the phase angle at the glass transition was plotted over the heater resistance and then the peak position $R_{T g}\left(f_{t h}\right)$, denoting the dynamic glass transition temperature, was determined for each frequency $f_{t h}$. From the VFT equations for toluene [115] and ethylbenzene [129] the corresponding temperatures can be determined under the assumption that dielectric and calorimetric responses give the same glass transition temperatures [130]. The inset in Figure 45 shows the cyclopentane calibration together with the two additional calibrations of toluene and ethylbenzene. The three calibrations are consistent with each other within $\pm 1 \mathrm{~K}$. This confirms, with the same uncertainty, the 
assumption of equal dynamic glass transition temperatures determined from the frequency dependent heat capacity and the dielectric permittivity for toluene and ethylbenzene. Since the three calibrations are consistent and performed independently, we trust the temperature measurements to $\pm 1 \mathrm{~K}$. We apply the calibration done with the cyclopentane transitions for the temperature determination since this calibration covers a broader temperature range and can be extrapolated to lower temperatures more reliably.

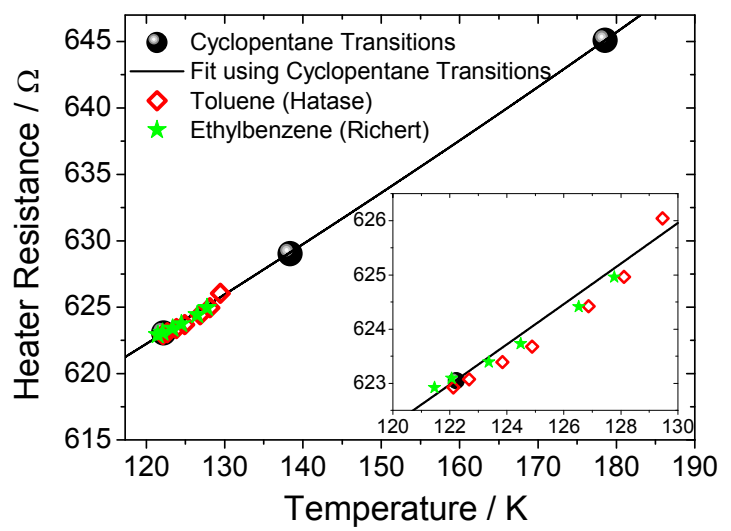

Figure 45: Determined calibration points for the three cyclopentane transitions (black dots) together with a second-order polynominal fit function (black line). Red diamonds and green stars represent the calibration points from the dynamic glass transition for different frequencies for toluene and ethylbenzene, respectively. The inset magnifies the temperature range between $120 \mathrm{~K}$ to $130 \mathrm{~K}$.

The reliability of extrapolation of the obtained calibration function to lower temperatures is tested using ethylcyclohexane, a volatile substance having a $T_{g}$ at $100.5 \mathrm{~K}$. We use it for rechecking the extrapolated calibration function in the vicinity of $0.85 T_{g}$ of toluene and ethylbenzene. The VFT equation of ethylcyclohexane is taken from Mandanici et al. [116] Measurements at $20 \mathrm{~Hz}$ and $2 \mathrm{~Hz}$, which yield values of $R_{T g}\left(f_{t h}\right)$, have shown that the extrapolation is in good agreement with the dynamic glass transitions of ethylcyclohexane, which are at $109.75 \mathrm{~K}$ and $107 \mathrm{~K}$, respectively. The deviation is about $1 \mathrm{~K}$. This gives confidence for reliable extrapolation of the calibration function to at least $100 \mathrm{~K}$.

The heater resistance of the sensors is not absolutely constant over time. Heating the membrane above room temperature for hours, e.g., while baking the chamber, causes a significant shift in the heater resistance. This restricts the heating of the membrane to room temperature, but even at room temperature the resistance can slightly shift. To take this into account the measured value of the glass transition temperature at $20 \mathrm{~Hz}$ is compared with the expected value from the 
corresponding VFT function and used to correct the heater resistance of each thin film experiment using the ordinary glass transition scan for the material under investigation. This correction assumes the temperature coefficients of the heater resistance do not change if the absolute value changes. Moreover no shift in the heater resistance is expected to occur between first and second heating. Depending on how many times the chamber was baked and how long the sample sensor has been used the cumulative correction can be in the order of $\pm 0.5 \Omega$ which is approximately $\pm 2 \mathrm{~K}$.

Summarizing, the functional form of $R(T)$ is determined from an initial three point calibration using cyclopentane as the calibrant. For each sample measurement the absolute value of the resistance is adjusted for long time changes by the measured $T_{g}(20 \mathrm{~Hz})$ for the slowly cooled toluene/ethylbenzene glass, which is used as an internal calibrant. This two-step calibration procedure results in an estimated total uncertainty for the sample temperature of $\pm 1 \mathrm{~K}$.

\subsubsection{Stressed membrane}

The temperature of the membrane is determined by use of the heater resistance. This requires reliable heater resistance data. However, during a temperature scan of a stiff sample (e.g. a crystal or stable glass) that is in very good contact with the membrane, the heater resistance increasingly deviates from values determined by e.g. an empty measurement. We attribute this to generation of mechanical stress on the membrane due to the different thermal expansion coefficients of the membrane and the stiff sample. Softening of the sample (e.g. melting) allows the temperature induced mechanical stress to relax. However only the value of the relaxed membrane can be applied to the temperature calibration function since the calibration function was derived from the heater resistance of a stress-free membrane.

Figure 46 provides data of a heating scan of an ordinary glass. The data is from an experiment with a $400 \mathrm{~nm}$ thick toluene film which was scanned up to a temperature above the melting point. In the beginning of the shown data the toluene is in the SCL state. The peak in the DC voltage at about $400 \mathrm{~s}$ is attributed to crystallization and the sample becomes stiff. During further heating mechanical stress develops between the sample and the membrane and relaxes while the sample melts at about $550 \mathrm{~s}$. When the stress to the membrane relaxes, the heater resistance attains the normal value. 


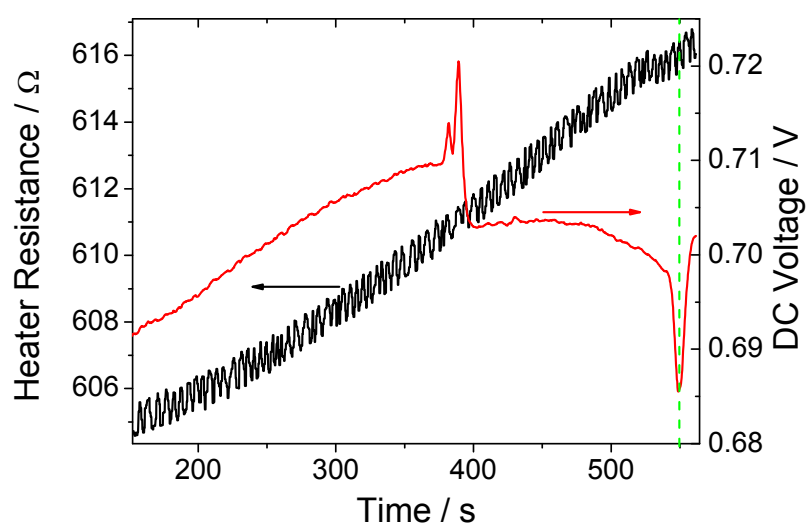

Figure 46: Heater resistance (black) and DC voltage (red) over time for heating a $400 \mathrm{~nm}$ film of toluene from the glass into the melt. The peak attributed as the melting peak (at $550 \mathrm{~s}$ ) is accompanied by an unusual behavior of the heater resistance. The heater resistance remains constant for about $10 \mathrm{~s}$ which can be addressed to softening of the sample (see text).

Unfortunately the membrane can also be stressed during temperature scans of stable glasses and solid cyclopentane. Particularly the first transition of cyclopentane is strongly influenced by this effect since the sample is deposited at about $105 \mathrm{~K}$ and crystallizes during deposition (crystallization interestingly occurs if a certain thickness is reached). Heating to the first transition $(\approx 122 \mathrm{~K})$ introduces high mechanical stress into the membrane so the heater resistance of the first transition cannot be determined from the as-measured data. However, the stress relaxes after the first transition. The heater resistance data of higher temperatures is extrapolated down to the temperature of the first transition of cyclopentane to obtain the nonstressed heater resistance for calibration purpose.

Stable glasses of toluene and ethylbenzene also introduce stress to the membrane. Here the stress relaxes if the stable glass transforms into the SCL. Again appropriate data treatment helps manage this effect.

Recently Lion et al. [131] published theoretical calculations on the influence of stress to membrane-suspended heat capacity measurements by fast scanning. Besides the initial temperature (e.g. deposition temperature) and the difference in the thermal expansion coefficients of the membrane and the sample, they found the heating rate to play a major role. They state the glass transition temperature is not affected by stress but the absolute heat capacity of the sample is. However, since our heating rates are far away from fast scanning rates we do not see and do not expect any influence to the heat capacity as shown in the work by Lion et al. 


\subsubsection{Thermopile voltage - to - temperature conversion}

One of the fundamental parameters in equation (33) to determine the heat capacity of the sample from the complex differential thermopile amplitude is the sensitivity $S$ of the thermopiles which represents the conversion factor between complex differential thermopile amplitude and complex differential temperature amplitude. Thus heat capacity cannot be determined without knowing this factor. However, equation (33) cannot be applied to determine the heat capacity of the sample unless other quantities like addenda heat capacity and temperature dependence of the addenda heat capacity are unknown. Nevertheless temperature amplitude is one of the required inputs for FEM. Thus the thermopile amplitude needs to be converted to temperature amplitude for fitting the model.

The measurement of the sensitivity is as follows: a known constant DC voltage is applied to the outer heater of the membrane of an empty single sensor. This is done with a function generator. The heater resistance of the inner heater of the membrane is measured as usual. The applied DC voltage is increased stepwise which leads to an increase of the membrane temperature $\left(T_{\text {substrate }}\right)$. The inner heater resistance is measured as a function of DC voltage. The independently determined temperature calibration function enables the calculation of $T_{\text {substrate }}$ from the heater resistance. The amplified and low-pass-filtered thermopile magnitude as measured at the SIGMON output of the lock-in amplifier with a DMM (see Appendix A.3) is also a measure of the temperature since it increases with the DC voltage applied. Thus temperature and preamplified thermopile voltage are brought into relation. After amplification-rectification of the preamplified thermopile voltage, which means the measured voltage is divided by the amplification, $T_{\text {substrate }}$ and the thermopile voltage can be plotted against each other (see Figure 47) which delivers the conversion between both parameters as the slope of the fit function in $\mathrm{mV} \mathrm{K}^{-1}$.

The DC voltage applied is varied from $0.0 \mathrm{~V}$ to $2.0 \mathrm{~V}$. Higher voltages may be possible but heating the membrane too much can cause the inner heater resistance to shift irreversibly, eliminating the current temperature calibration of the sensor. The oven temperature is $105 \mathrm{~K}$ during the entire experiment. The acquired data is shown in Figure 47 . Up to about $170 \mathrm{~K}$ the points follow a linear relation; for higher temperatures a deviation is observed due to enhanced overheating since the supplied heat cannot dissipate. Moreover, the temperature calibration function is only valid for temperatures up to $178 \mathrm{~K}$. Above the calculated temperature has to be extrapolated and the error grows with temperature. Hence only the linear regime of Figure $47 \mathrm{can}$ be used for sensitivity determination. A linear fit yields a slope of $1.3 \mathrm{mV} \mathrm{K}^{-1}$. This value is temperature dependent and will be different at e.g. room temperature. For the small temperature range that is needed for the current work the sensitivity is assumed to be constant. 


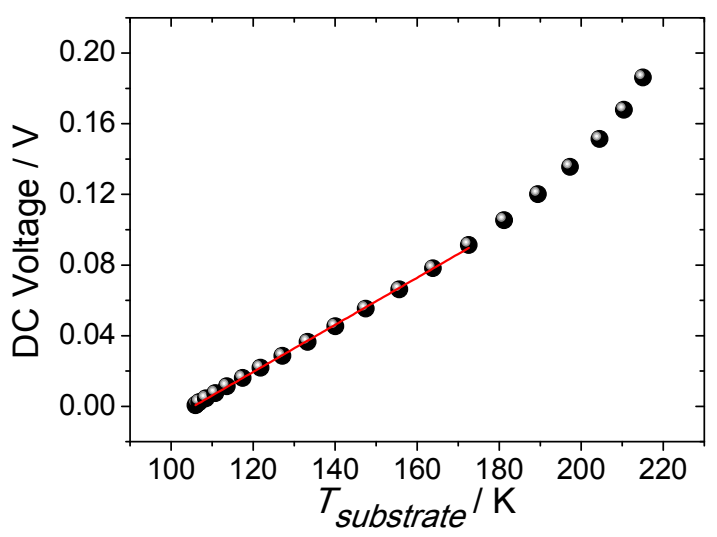

Figure 47: The determination of the sensitivity of the thermopiles of the used sensors. The linear relation between thermopile DC voltage and $T_{\text {substrate }}$ yields the sensitivity of the thermopiles of the used sensors which is about $1.3 \mathrm{mV} \mathrm{K}^{-1}$.

\subsection{Experimental implementation}

\subsubsection{Film thickness and deposition rate}

The thickness of the film that is commonly deposited onto the membrane of the sensor is held constant at $390 \mathrm{~nm}$. In terms of differential thermopile magnitude this corresponds to $12.7 \mu \mathrm{V}$ at $f_{t h}=10 \mathrm{~Hz}$ (see section 3.2.4). Before the sensor shutter that protects the sample sensor from uncontrolled deposition is opened, the deposition rate is adjusted to about $2 \mathrm{~nm} \mathrm{~s}^{-1}$. When the desired film thickness is reached the shutter valve closes automatically. Figure 48 shows a deposition process over time. The plot shows the evolution of the magnitude and the phase of the complex differential temperature amplitude. The inset shows the thickness and the deposition rate over time as determined with the QCM. It can be seen that the linear increase in the film thickness as measured by the QCM matches an almost linear increase in the differential temperature amplitude. This indicates a close to linear relationship between the measured sample heat capacity and the film thickness of the sample (which is only valid up to a film thickness of about $500 \mathrm{~nm}$, see section 3.2.4). The phase increases by about $15^{\circ}$. 


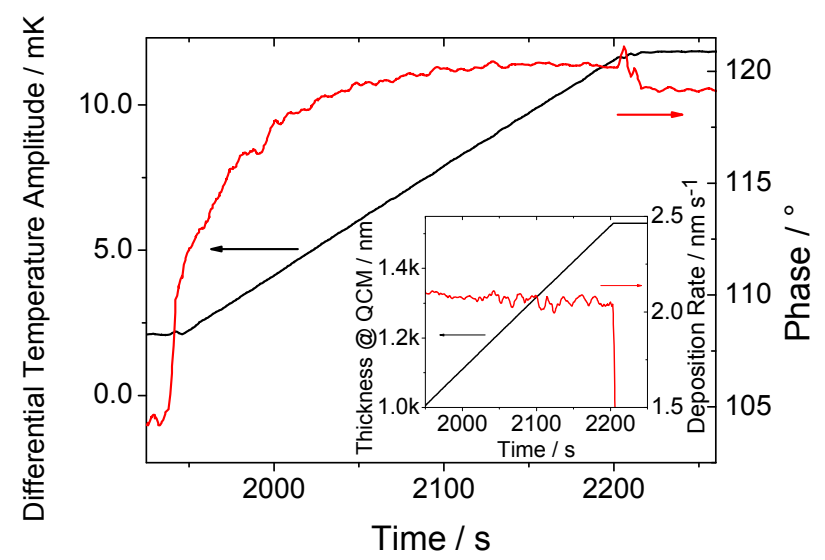

Figure 48: Differential temperature amplitude (black) and phase (red) during a deposition process at $105 \mathrm{~K}$. The linear increase of the film thickness as measured with the QCM is mirrored by the nearly linear increase of the differential temperature amplitude.

\subsubsection{Temperature programs}

Depending on the experiment, isothermal or temperature ramping measurements using a frequency $f_{t h}=20 \mathrm{~Hz}$ (see section 3.2.1) follow the deposition. Since toluene and ethylbenzene tend to crystallize slightly above the dynamic glass transition temperature the maximum temperature of the temperature ramping has to be chosen carefully. Moreover the formation of nuclei during heating/cooling cycles or with annealing time allows the sample to crystallize at even lower temperatures.

To avoid crystallization the first heating of the stable glass is often scanned only up to a temperature where the dynamic glass transition is not fully finalized. Particularly this is the case for toluene since it crystallizes easily. Since a second heating scan of a completely ordinary glass is essential for further data treatment it is necessary that the sample fully transforms from the stable glass to the SCL before it is cooled down again. Fortunately the transformation from the stable glass to the SCL starts slightly below the $20 \mathrm{~Hz}$ glass transition temperature for toluene and finishes (depending on the heating rate) slightly above $T_{g, d y n}$. This fact allows for successful prevention of crystallization. Usually the second heating can be performed up to the crystallization temperature and higher as long as no subsequent heating scans will follow. The used temperature program, which represents a normal scan experiment including $1^{\text {st }}$ and $2^{\text {nd }}$ heating, is shown in Figure 49. 


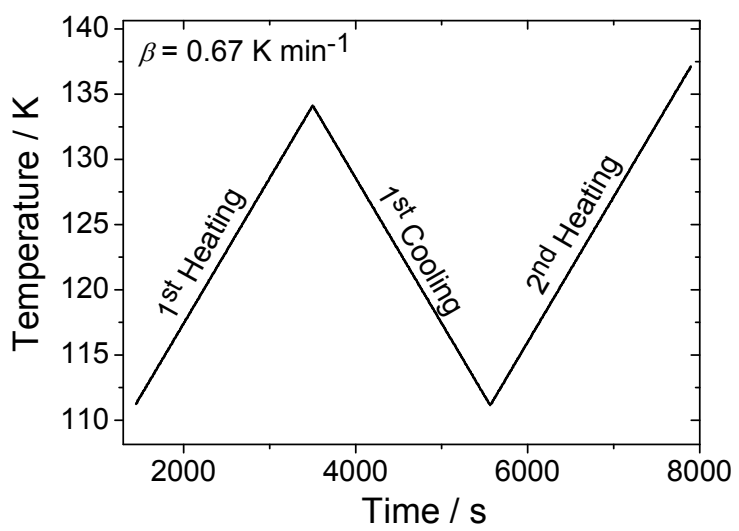

Figure 49: Temperature program for the dynamic glass transition scans of toluene. The heating rate $\beta$ is small to ensure controlled heating of the copper block. The maximum temperature of the first heating is carefully chosen to avoid crystallization of the sample. The second heating scans to higher temperature to encompass the full dynamic glass transition.

In isothermal transformation experiments, attention is paid to the maximum transformation temperature. The higher the transformation temperature is the smaller the transformation time will be. If the transformation temperature is set too high the transformation will start and proceed even if the set transformation temperature is not reached by the system. The maximum transformation temperature is an interplay between the time constant of the temperature control and the transformation speed.

After successful transformation the sample is cooled down to the initial temperature to retain the same initial conditions prior to the temperature ramping as for all other experiments. 


\section{In-situ measurements of vapor-deposited toluene and ethylbenzene}

In the following chapter the stability of vapor-deposited glasses of toluene and ethylbenzene as a function of substrate temperature and deposition rate is investigated by in-situ AC calorimetry. Moreover the kinetics of the transformation of the stable glass to the SCL is investigated as a function of transformation temperature and film thickness.

\subsection{Detection limit for heat capacity and a possible mobile layer}

The minimum sample heat capacity that can be detected with the current device is a question of signal-to-noise ratio. By scanning the glass transition several times with subsequent data averaging we were able to detect the glass transition of a $2 \mathrm{~nm}$ thin film of toluene (see Figure 50). The film thickness was determined by downscaling the result of the FEM to the deposited differential thermopile magnitude of $80 \mathrm{nV}$.

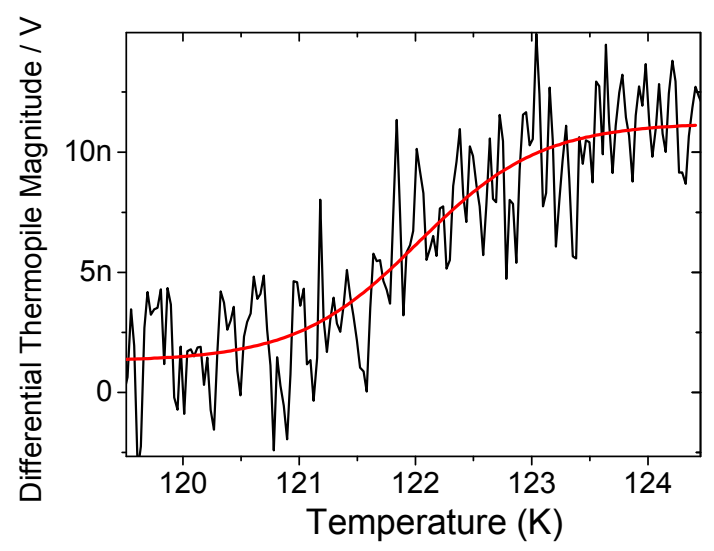

Figure 50: The glass transition of a $2 \mathrm{~nm}$ thin film of toluene. Since the signal-to-noise ratio is not sufficient to resolve the glass transition of such a thin film the shown data (black) represents an average of about 8 heating scans of the glass transition. The red line is a fit for better visualization of the glass transition step. For easier data manipulation and to correct for possible signal shift during the eight heating scans the heating curves were set to zero in the glassy state at $T_{\text {substrate }}=110 \mathrm{~K}$.

The glass transition step height $\Delta C_{p}$ of the $2 \mathrm{~nm}$ thick film in terms of differential thermopile voltage is about $10 \mathrm{nV}$ which is only $12 \%$ of the value in the glassy state $(80 \mathrm{nV})$. Yamamuro et al. [111] provide $\Delta C_{p}$ to be about $80 \%$ of the glassy $C_{p}$ for bulk toluene at 
$T_{g}=117 \mathrm{~K}$. At $T_{g, d y n}(20 \mathrm{~Hz})=122 \mathrm{~K}$ this ratio decreases to $66 \%$ because of the different slopes of the glass and the liquid $c_{p}$. Additionally, the non-linear dependence between the heat capacity and the differential temperature amplitude (Figure 51 (b)) in the AC calorimetric measurements causes a further reduction of $\Delta C p$. The expected ratio for $\Delta C_{p} / C_{p}$ as function of film thickness is shown in Figure 51 (a) as a red line. Figure 51 shows data for ordinary glasses that were obtained from samples with film thicknesses ranging from $2 \mathrm{~nm}$ to $200 \mathrm{~nm}$, deposited at a substrate temperature of $105 \mathrm{~K}$ and a deposition rate of $2 \mathrm{~nm} \mathrm{~s}^{-1}$. The as-deposited samples were heated to $\sim 125 \mathrm{~K}$ to ensure that they were in the supercooled liquid state. Next, the samples were cooled at $0.67 \mathrm{~K} \mathrm{~min}^{-1}$ to $105 \mathrm{~K}$ to form a slowly cooled ordinary glass. The data presented in Figure 51 were obtained from the second heating to $125 \mathrm{~K}$. Subsequent heating scans were performed for the thinnest films (below $10 \mathrm{~nm}$ ) because the glass transition was only visible after averaging several heating curves. Film thickness was controlled by deposition time and it was assumed that a homogeneous film was present (no dewetting). It can be seen that for a film thickness of about $25 \mathrm{~nm}$ the ratio $\Delta C_{p} / C_{p}$ (glass) already decreases significantly below the expected value.
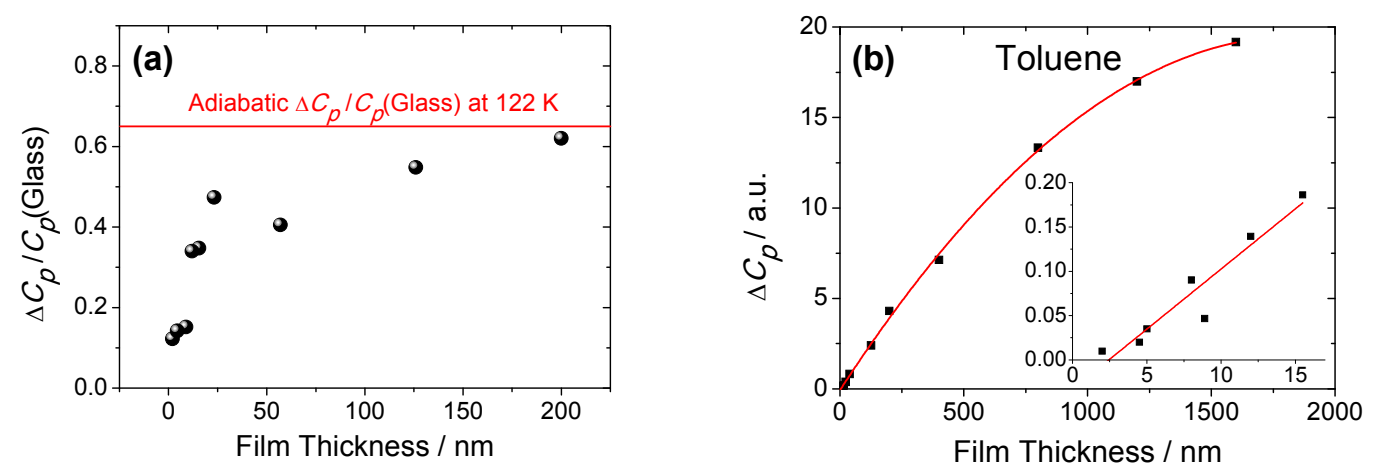

Figure 51: (a) $\Delta C_{p} / C_{p}$ (glass) over film thickness for different depositions of thin films of ordinary glasses of toluene. The ratio decreases with decreasing film thickness possibly due to the influence of a mobile layer. The red line represents the value determined from adiabatic data [111]. (b) Thickness dependence of the step height at the glass transition from the measured differential temperature amplitude for ordinary glasses of toluene. The inset in (b) shows the data for films thinner than $16 \mathrm{~nm}$. The red lines in (b) are fits, see text.

Recent investigations of thin films of stable glass forming materials gave evidence of a mobile surface layer that does not contribute to the glass transition [66, 67]. Assuming the mobile layer thickness is the same for thick and thin films [67] its portion by percentage of the sample increases by going to very thin films. Thus the ratio $\Delta C_{p} / C_{p}$ (glass) decreases for such thin films and the mobile layer has considerable impact on the decrease in $\Delta C_{p}$ as seen in Figure 51 (a). The 
step height of the measured heat capacity at $T_{g, d y n}$ is shown. The nonlinear overall behavior is due to the nonlinear relationship between the measured signal and the heat capacity [13]. The inset shows the data for the thinnest films; the linear fit to the data extrapolates to zero step height at $\approx 2 \mathrm{~nm}$. This may indicate a surface layer that is not participating in the glass transition due to higher mobility.

Besides the step height the experiments at small film thicknesses revealed the dynamic glass transition temperature to be independent of film thickness in accordance to other works [89, 132, 133] (Figure 52). On the contrary, many publications proved a glass transition temperature dependence on film thickness $[61,134]$. However, this contradiction is still unsolved and under heated debate.

Our results confirm that the $390 \mathrm{~nm}$ thick films used in our experiments are not influenced by confinement effects. Even if some dewetting occurred and the film evolved into thicker droplets, a serious $T_{g, d y n}$ depression is not observed.

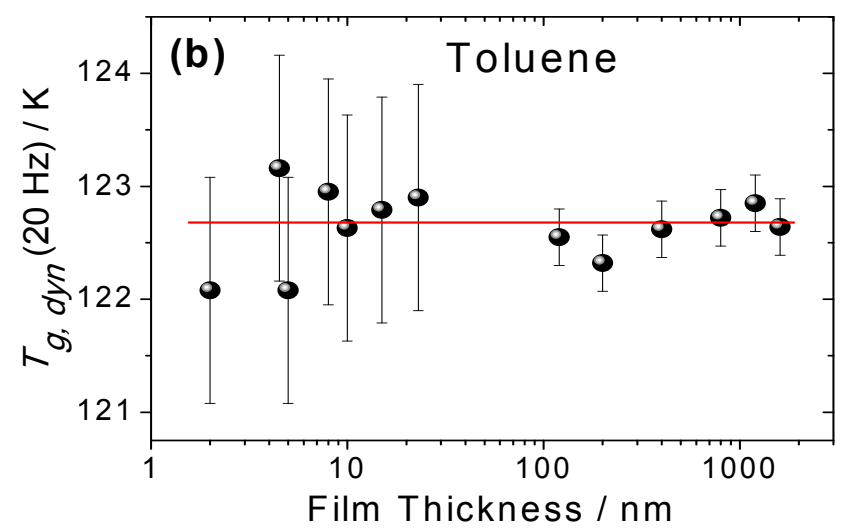

Figure 52: Thickness dependence of the dynamic glass transition temperature at $20 \mathrm{~Hz}$. The red line represents the mean value.

\subsection{Stability of as-deposited glasses}

As mentioned in section 2.2.3 the thermodynamic and kinetic stability of vapor-deposited glasses is highly dependent on the substrate temperature (deposition temperature). Using deposition temperatures far below the glass transition temperature have led to the assumption that vapor deposition yields glasses with high enthalpy and thus high fictive temperatures [39]. However, recently deposition temperatures close to the glass transition temperature were discovered to yield kinetically highly stable glasses with low fictive temperatures [5]. This is a contradiction that potentially can be solved by bridging the gap between low temperature and 
close-to- $T_{g}$ depositions and thus taking a closer look to the stable glass formation process during deposition.

In this work, AC nanocalorimetry is used to quantify the stability of vapor-deposited glasses of toluene and ethylbenzene. Glasses of the two molecules were deposited over a range of substrate temperatures and deposition rates in order to determine the conditions that yield the most stable vapor-deposited glasses. In these measurements, there are two features of the heat capacity curves that can be used to quantify stability: the heat capacity of the as-deposited glass and the onset temperature for the transformation of the as-deposited glass into the supercooled liquid.

Thin films $(390 \mathrm{~nm})$ of toluene and ethylbenzene were vapor-deposited at constant deposition rate $\left(2 \mathrm{~nm} \mathrm{~s}^{-1}\right)$ but different substrate temperatures varying between $71 \mathrm{~K}$ and $65 \mathrm{~K}$ and the dynamic glass transition temperature of toluene and ethylbenzene, respectively. In terms of the glass transition temperature of toluene, the temperature range is from $0.61 T_{g}$ to $1.03 T_{g}$; in terms of the glass transition temperature of ethylbenzene, the range is from $0.56 T_{g}$ to $1.03 T_{g}$. All other experimental parameters were kept constant $\left(\beta=0.67 \mathrm{~K} \min ^{-1}, \quad f_{t h}=20 \mathrm{~Hz}\right.$, film thickness $=390 \mathrm{~nm}$ ). Film thickness is reproduced within $\pm 5 \%$ for deposition rates below $10 \mathrm{~nm} \mathrm{~s}^{-1}$ even though the absolute value is only defined as $390 \pm 80 \mathrm{~nm}$ [13]. Since the heating scan of a stable glass induces an irreversible transformation, each measurement requires a separately deposited sample. For the current investigation about 50 samples were deposited. For this large number of samples, film thickness scatters around the target value. To allow direct comparison of the data from samples with slightly different thicknesses, the amplitude of the measured curves for the second heating are normalized at $113 \mathrm{~K}$; the same normalization factor is also applied to the corresponding first heating scan. This corrections lead to $2^{\text {nd }}$ heating curves that lie on top of each other and ideally showing the same glassy and liquid reversing heat capacity and thus the same $\Delta C_{p}$.

All measurements have to be consistent regarding the temperature. Therefore the phase peak of the ordinary glass transition ( $2^{\text {nd }}$ heating scan) of each experiment was used to correct the heater resistance data of the corresponding experiment regarding a reference value which was determined directly after temperature calibration. Since the measurements are done at $20 \mathrm{~Hz}$ the heater resistance of the phase peak corresponds to $122.66 \mathrm{~K}$ for toluene and $122.06 \mathrm{~K}$ for ethylbenzene as determined from the corresponding VFT [115, 129].

Figure 53 shows the baseline-corrected $1^{\text {st }}$ heating scans for as-deposited glasses of toluene and ethylbenzene. For comparison, the dotted line shows the heat capacity curve of an ordinary glass (second heating). The shown data is measured with the LN2-cooled device only. Additional measurements were done for lower deposition temperatures with the He-cooled device and are 
shown in Figure 54. All measured curves were scaled as described above. This scaling allows the glassy $C_{p}$ of the different stable glasses to be compared even though the absolute heat capacity values are not known.

Figure 53 and Figure 54 show that the heat capacity of vapor-deposited glasses is different than that of liquid-cooled glasses. Except for glasses deposited very close to $T_{g}$, the as-deposited glasses have lower reversing heat capacity than the ordinary glass, as can be observed by comparison at $114 \mathrm{~K}$. The low heat capacity is maintained until the transformation to the supercooled liquid occurs, well above the glass transition temperature $T_{g}$. As the transformation is completed, the reversing heat capacity finally joins the same curve observed when an ordinary glass is heated. Note that AC calorimetry measures the reversing heat capacity (modulus of complex heat capacity) and thus no enthalpy relaxation peak is observed.

The characterization regarding the low enthalpy of stable glasses was introduced by Kearns et al. as the drop in fictive temperature compared to an ordinary glass [5]. Because fictive temperature is not available from the reversing heat capacity it cannot be determined here. However, AC calorimetry does provide small differences in the heat capacity between stable and ordinary glasses [51]. The lower heat capacity of the stable glass, as seen in Figure 53 and Figure 54, can be linked to the vibrational degrees of freedom which may be linked to the packing of the glass [45]. We utilize the fractional $C_{p}$ decrease to characterize the difference in heat capacity between stable and ordinary glasses:

$$
\text { Fractional } C_{p} \text { decrease }=1-\frac{C_{p}(S G)}{C_{p}(O G)}
$$

where SG refers to "stable glass" and OG refers to "ordinary glass". For the measurements here, the fractional $C_{p}$ decrease was calculated at $113 \mathrm{~K}$, which is $T_{g}-4 \mathrm{~K}$ and $T_{g}-2 \mathrm{~K}$ for toluene and ethylbenzene, respectively. This temperature is low enough to ensure that we were directly comparing the solid-state properties of the as-deposited and ordinary glass.

We do not use the method proposed by Kearns et al. that compares the $C_{p}$ step at the glass transition of stable glasses with that of ordinary glasses by $\frac{\Delta C_{p}(S G)}{\Delta C_{p}(O G)}[51]$. Even though this method has the big advantage that baseline correction is not necessary, it is difficult to apply for crystallizing materials like toluene. Furthermore $\Delta C_{p}$ is often more material specific than the heat capacity in the glassy state.

The onset temperature for the transformation into the supercooled liquid is an important measure of the kinetic stability of the as-deposited glasses. The higher the temperature required to dislodge the molecules from the glass, the greater the kinetic stability of the as-deposited material. As shown by the solid black lines in Figure 53, the onset temperature is determined from the 
reversing heat capacity as the intersection of a tangent to the leading edge of the heat capacity increase near $T_{g, d y n}$ and the extrapolated heat capacity of the corresponding glasses [34]. The steep increase in reversing heat capacity above $T_{g}$ is caused by the transformation of the stable glass into the supercooled liquid. For much lower modulation frequencies (much lower $T_{g, d y n}$ ) the transformation would occur above $T_{g, d y n}$ and result in an even larger change of the reversing heat capacity; the amplitude of this change would be independent of the transformation temperature. In our case $\left(f=20 \mathrm{~Hz}, T_{g, d y n}=122.7 \mathrm{~K}\right.$ and 122.1 for toluene and ethylbenzene, respectively) the transformation occurs in the temperature region of the dynamic glass transition and the step height is therefore dependent upon the transformation temperature.
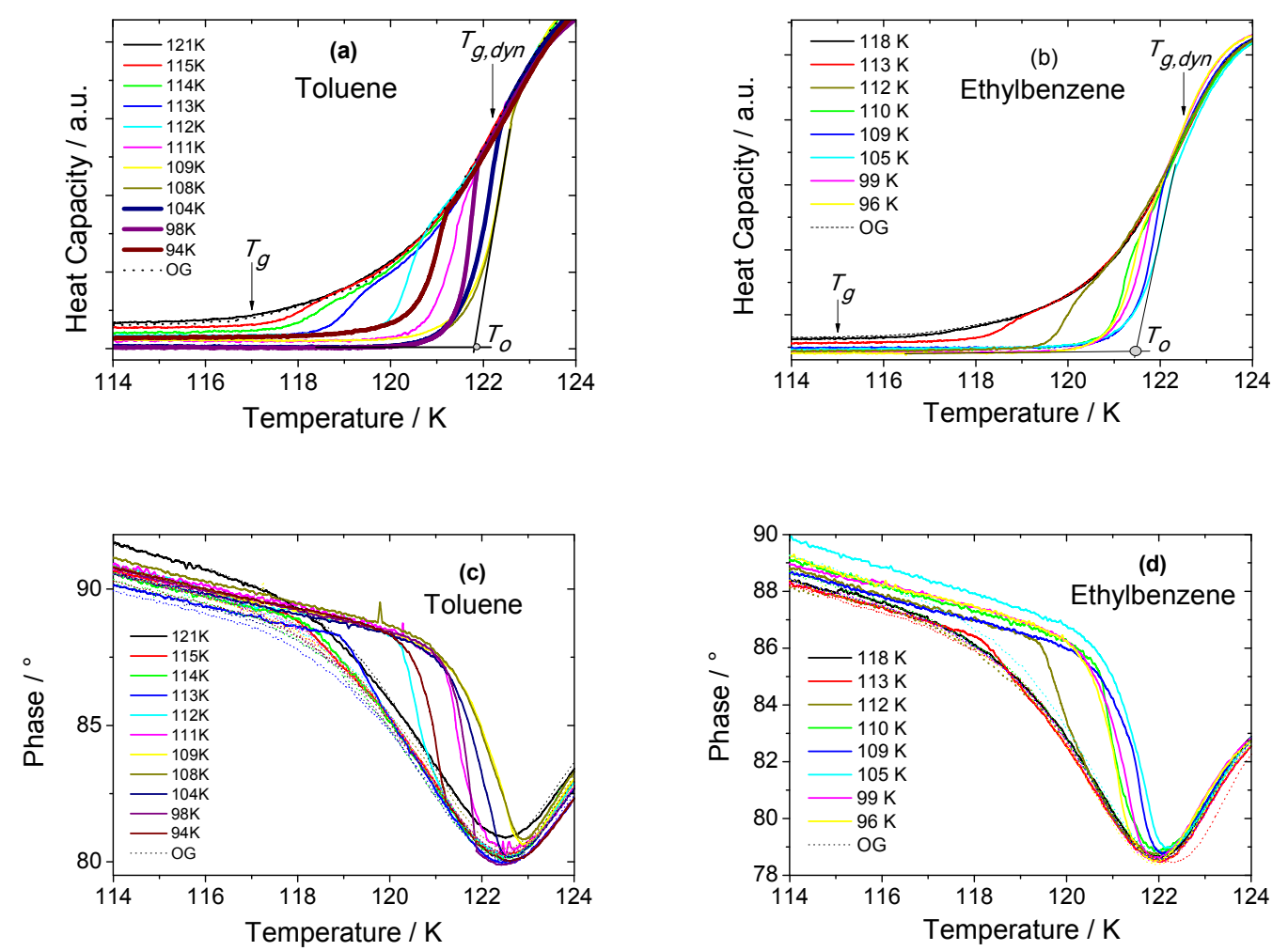

Figure 53: Heat capacity of the first heating curves of as-deposited stable glasses of (a) toluene deposited at substrate temperatures ranging from $94 \mathrm{~K}$ to $121 \mathrm{~K}$ and (b) ethylbenzene deposited from $96 \mathrm{~K}$ to $118 \mathrm{~K}$ measured with the LN2-cooled device. (c) and (d) show the corresponding phase data. The $121 \mathrm{~K}$ deposition of toluene as well as the $118 \mathrm{~K}$ deposition of ethylbenzene shows no extraordinary stability since the deposition temperatures are above the glass transition of toluene and ethylbenzene, respectively. For comparison a heating curve of the ordinary glass is shown for both materials. All films were deposited with a rate of $2 \mathrm{~nm} \mathrm{~s}^{-1}$ and the film thickness was held constant at about $390 \mathrm{~nm}$. Heating scans were performed with a rate of $0.67 \mathrm{~K} \mathrm{~min}^{-1}$ and a frequency of $20 \mathrm{~Hz}$. 

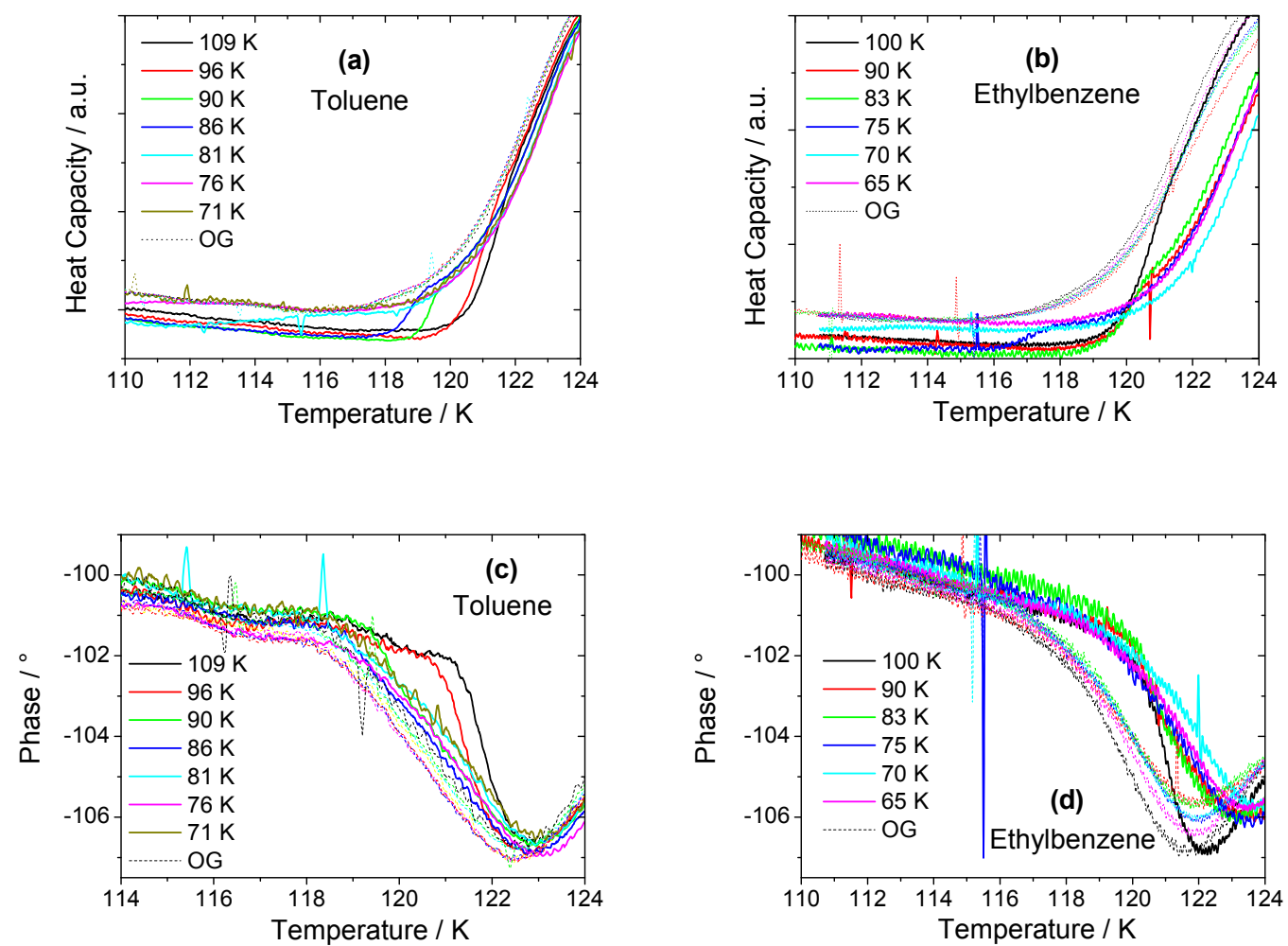

Figure 54: Heat capacity of the first heating curves of as-deposited stable glasses of (a) toluene deposited at substrate temperatures ranging from $71 \mathrm{~K}$ to $109 \mathrm{~K}$ and (b) ethylbenzene deposited from $65 \mathrm{~K}$ to $100 \mathrm{~K}$ measured with the He-cooled device. (c) and (d) show the corresponding phase data. For comparison the heating curves of the corresponding ordinary glasses are shown for both materials. All films were deposited with a rate of approx. $2 \mathrm{~nm} \mathrm{~s}^{-1}$ and the film thickness was held constant at about $390 \mathrm{~nm}$. Heating scans were performed with a rate of $0.67 \mathrm{~K} \mathrm{~min}^{-1}$ and a frequency of $20 \mathrm{~Hz}$.

\subsection{Stability depending on substrate temperature}

For each as-deposited glass in Figure 53 and Figure 54, the onset temperature was determined as shown and the fractional $C_{p}$ decrease was calculated according to equation (36). These values are compiled in Figure 55. The onset temperature for the transformation from the stable glass to the supercooled liquid follows the same trend for toluene and ethylbenzene (Figure 55 (a) and Figure 55 (b), respectively). When the substrate temperature is above $T_{g}$ an ordinary glass is deposited and the onset temperature is about $6 \mathrm{~K}$ and $4 \mathrm{~K}$ below $T_{g, d y n}$ for toluene $\left(T_{g, d y n}=122.7 \mathrm{~K}\right)$ and ethylbenzene $\left(T_{g, d y n}=122.1 \mathrm{~K}\right)$, respectively. The onset temperature, $T_{o n}$, increases for lower deposition temperatures until a maximum is reached which is $1 \mathrm{~K}$ below $T_{g, d y n}$ for both compounds. This maximum holds for a range of substrate temperatures until $T_{\text {on }}$ slightly 
drops for deposition temperatures below $0.83 T_{g}$ and $0.85 T_{g}$ for toluene and ethylbenzene, respectively, and continues dropping all the way to $0.6 T_{g}$. Here the onset temperature, determined from the slight step in the heat capacity curve, drops below the glass transition temperature. We speculatively identify the very low onset temperatures with the deposition of unstable glasses, in contrast with the stable glasses prepared at high substrate temperatures, see discussion in section 5.2.

The fractional $C_{p}$ decrease, which is determined by the ratio of the glassy $C_{p}$ of the stable and the ordinary glasses, follows a similar trend as the onset temperature. At substrate temperatures very close to $T_{g}$ vapor-deposited glasses exhibit no detectable heat capacity decrease for both toluene and ethylbenzene; in this case the vapor-deposited glass is indistinguishable from a liquid-cooled glass. For lower substrate temperatures the $C_{p}$ of the vapor-deposited glasses drops by $4 \%$ as compared to the ordinary glass. This maximal drop is seen for glasses of toluene vapordeposited at substrate temperatures between $0.69 T_{g}$ and $0.92 T_{g}$, and for glasses of ethylbenzene between $0.65 T_{g}$ and $0.96 T_{g}$. For temperatures below these ranges the heat capacity decrease diminishes gradually in both systems.
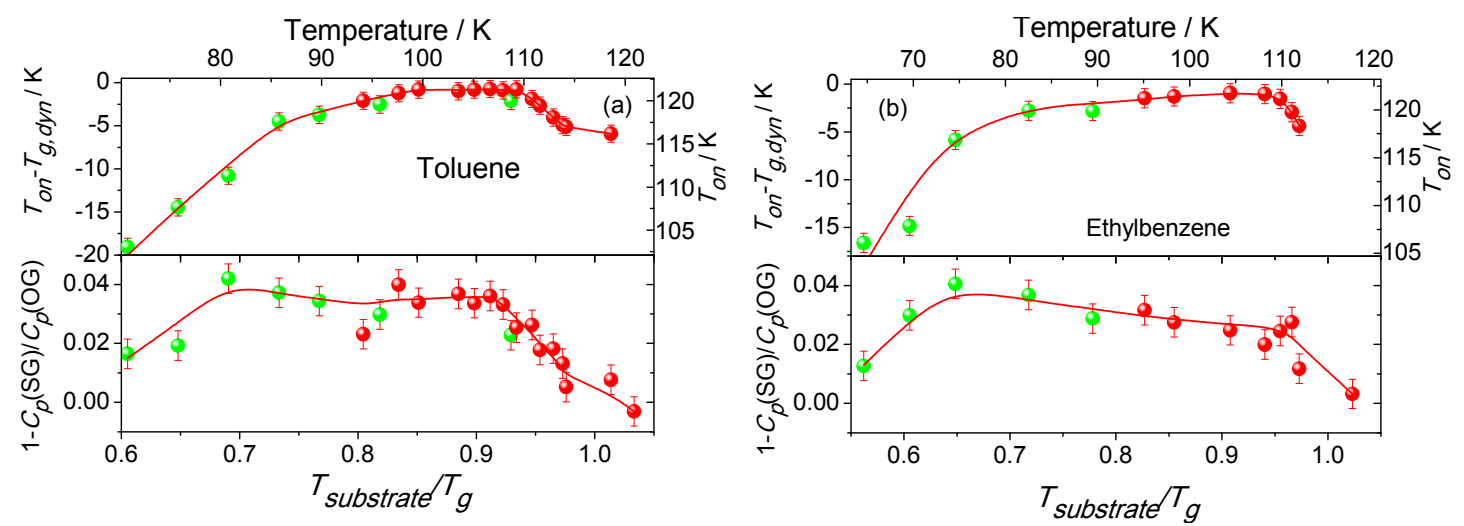

Figure 55: Onset temperature $\left(T_{o n}\right)$ and fractional $C_{p}$ decrease of as-deposited stable glasses of (a) toluene and (b) ethylbenzene for different substrate temperatures as determined from Figure 53 and Figure 54. The substrate temperature is normalized by the glass transition temperature of the corresponding molecule. Red dots represent data determined with the LN2-cooled device, while green dots are data determined with the He-cooled device. The error bars in the upper panel of both figures represent the absolute accuracy of temperature determination whereas the error bars in the lower panel represent the standard deviation due to the noise of the differential thermopile signal.

Figure 54 may give the impression that the temperature determination somehow failed since the dynamic glass transition temperatures of the $1^{\text {st }}$ heating and $2^{\text {nd }}$ heating are well 
separated. This shift in the dynamic glass transition temperature for the samples deposited at very low deposition temperatures is real and no artifact of the measurement. This feature is investigated in detail in section 4.6.

\subsection{Stability depending on deposition rate}

The influence of the deposition rate on the stability of vapor-deposited glasses of toluene and ethylbenzene was determined by varying the deposition rate by more than three orders of magnitude from $0.02 \mathrm{~nm} \mathrm{~s}^{-1}$ to $50 \mathrm{~nm} \mathrm{~s}^{-1}$. Figure 56 shows the measured curves for the stable glasses; the substrate temperature was $105 \mathrm{~K}$ in all measurements, which is $0.9 T_{g}$ and $0.91 T_{g}$ for toluene and ethylbenzene, respectively. Aside from the deposition rate, all other experimental parameters were kept constant $\left(\beta=0.67 \mathrm{~K} \mathrm{~min}^{-1}, f_{t h}=20 \mathrm{~Hz}\right.$, film thickness $\left.=390 \mathrm{~nm}\right)$ and equal to those used in the measurements shown in Figure 53 and Figure 54. The temperature scanning program is exactly the same as for the substrate dependent measurements (see section 4.2). The ordinary liquid-cooled glasses are also shown in Figure 56 for comparison.

Compared to the substrate temperature dependence the changes due to deposition rate variation are much weaker as summarized in Figure 57. For toluene, deposition at $50 \mathrm{~nm} \mathrm{~s}^{-1}$ gives a fractional $C_{p}$ decrease of about 0.025 and for ethylbenzene 0.01 as compared to the ordinary glass. Higher deposition rates cannot be realized since the $50 \mathrm{~nm} \mathrm{~s}^{-1}$ deposition requires only $8 \mathrm{~s}$ and well-defined films are difficult to produce at higher rates (shorter times). The difference in $T_{o n}$ between the stable glasses of toluene deposited at different rates is only $0.4 \mathrm{~K}$, which is within the uncertainty of absolute temperature determination. Slightly larger increases are observed for ethylbenzene. At lower deposition rates, a small but significant decrease in heat capacity is detectable in Figure 56. 

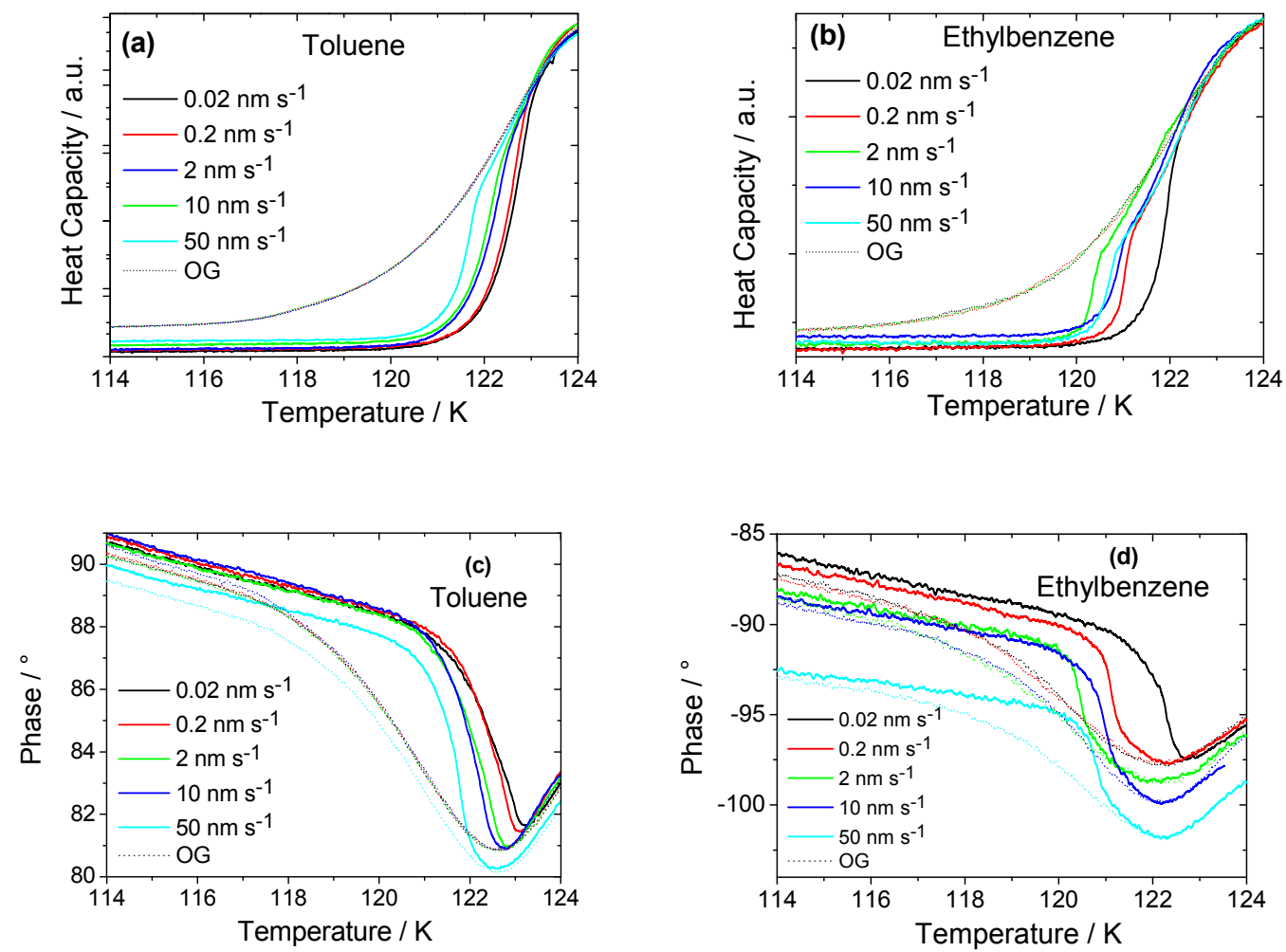

Figure 56: Heat capacity of as-deposited stable glasses of (a) toluene and (b) ethylbenzene deposited at deposition rates spanning more than three orders of magnitude. (c) and (d) show the corresponding phase data. For comparison, the heating curves of the corresponding ordinary glass are shown as dotted lines. All films were deposited at a substrate temperature of $105 \mathrm{~K}$ and the film thickness was held constant at about $390 \mathrm{~nm}$. Heating scans were performed with a rate of $0.67 \mathrm{~K} \mathrm{~min}^{-1}$ and at a frequency of $20 \mathrm{~Hz}$.
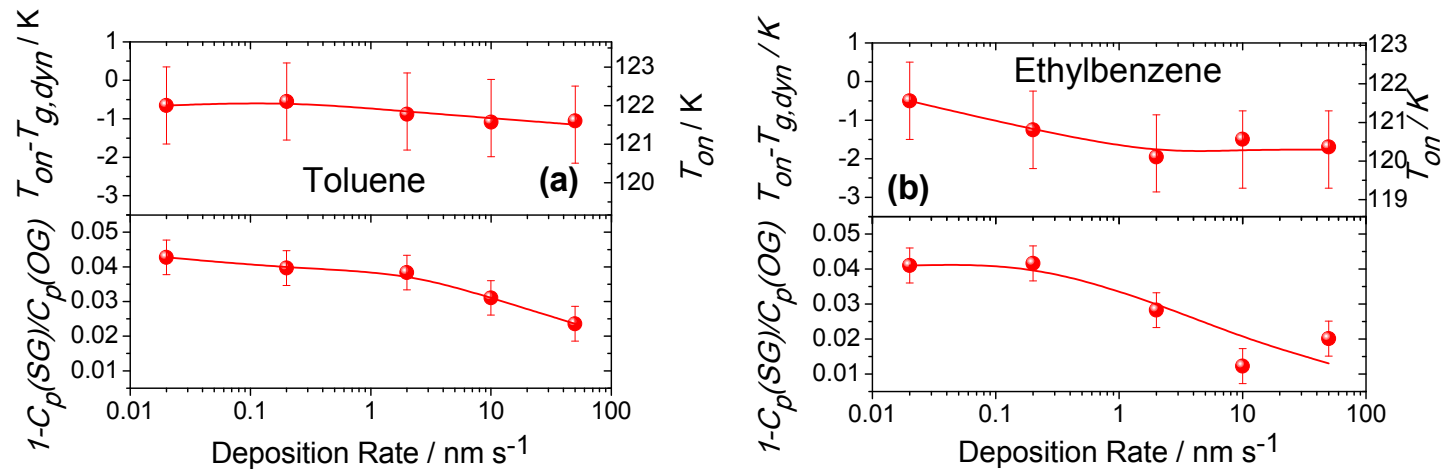

Figure 57: The onset temperature $\left(T_{o n}\right)$ and fractional $C_{p}$ decrease of as-deposited stable glasses of (a) toluene and (b) ethylbenzene for different deposition rates, as determined from Figure 56. 
Even though a rate dependence can be observed in Figure 56 and Figure 57, the effect is weak in comparison to the changes induced by the substrate temperature variation.

\subsection{Transformation time dependence on temperature and film thickness}

The kinetic stability of high stability glasses can be more accurately measured by observing the transition to the supercooled liquid at quasi-isothermal conditions [51]. This is possible via AC calorimetry because at all temperatures the heat capacity of the stable glass is lower than that of the supercooled liquid or ordinary glass [51]. In such experiments, the reversing heat capacity is observed to slowly increase from the stable glass value to the equilibrium value.

For toluene and ethylbenzene the transformation of the stable glass into the SCL was followed for different transformation temperatures $T_{\text {transform}}$, while holding the film thickness constant at about $390 \mathrm{~nm}$. $T_{\text {transform }}$ was varied by $3 \mathrm{~K}$ for toluene and $2 \mathrm{~K}$ for ethylbenzene and the lowest transformation temperature was $2 \mathrm{~K}$ above $T_{g}$ of the substances.

To simplify the comparison of different transformation experiments Kearns et al. [45] proposed to calculate the fraction of stable glass $\Phi_{S G}(t)$ at any given time by

$$
\Phi_{S G}(t)=\frac{C_{p}(O G)-C_{p}(S G, t)}{C_{p}(O G)-C_{p}(S G, t=0)} .
$$

Thus $\Phi_{S G}(t)=1$ for pure stable glass at $t=0$ and $\Phi_{S G}(t)=0$ if the sample is fully transformed into the SCL state. The time that the as-deposited sample needs to transform fully is denoted as the transformation time $t_{\text {transform }}$.

The time required for the stable glass to transform into the supercooled liquid is very sensitive to the transformation temperature. Figure 58 shows $\Phi_{S G}(t)$ for different annealing temperatures for toluene (a) and ethylbenzene (b). The linear scale of the time-axis makes it hard to see the fastest transformations but a linear representation is chosen to highlight the linear behavior of the transformation process. A change of $2 \mathrm{~K}$ in $T_{\text {transform }}$ changes the transformation time by a factor of 22 for toluene and by a factor of 10 for ethylbenzene. As will be discussed below, the linear transformation process is consistent with a surface-initiated growth front. 

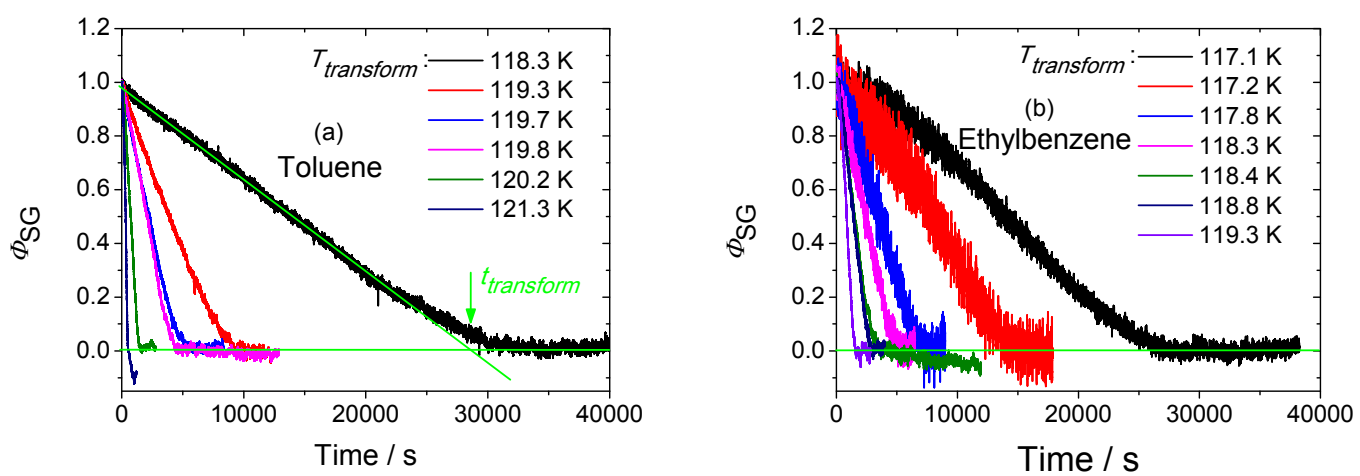

Figure 58: Isothermal transformation kinetics of stable glasses of (a) toluene and (b) ethylbenzene to the SCL for different transformation temperatures. The y-axis is $\Phi_{S G}(t)$ which indicates the fraction of the sample with the heat capacity of the stable glass. All samples were deposited at a substrate temperature of $105 \mathrm{~K}$ and a deposition rate of $2 \mathrm{~nm} \mathrm{~s}^{-1}$. Film thickness is $390 \mathrm{~nm}$. The larger scatter observed for lower transformation temperatures is due to the smaller $C_{p}$ difference between the stable glass and the supercooled liquid.

The data shown in Figure 58 allow a comparison of the transformation times with the structural relaxation time $\left(\tau_{\alpha}\right)$ of the SCL; $\tau_{\alpha}$ also approximately represents the time required for an ordinary glass to transform into the supercooled liquid. In order to compare different materials, in Figure 59 the logarithm of the ratio $\frac{t_{\text {transform }}}{\tau_{\alpha}}$ is plotted versus the negative of the logarithm of the structural relaxation time. Please note that the $\mathrm{x}$-axis is set as $-\log \left(\tau_{\alpha}\right)$ and thus the temperature that determines the structural relaxation time can be read as increasing from left to right on the $\mathrm{x}$ axis.

The points for toluene, ethylbenzene and indomethacin (IMC) seem to follow the same trend and the stable glasses show comparable stability. All three systems show a sub-linear dependence of $t_{\text {transform }}$ on $\tau_{\alpha}$, as qualitatively anticipated by Wolynes for a SCL that exhibits spatially heterogeneous dynamics [135]. The temperature dependence of $t_{\text {transform }}$ is observed to be weaker than that of $\tau_{\alpha}$. The plot also highlights the transformation time exceeds $10^{3.8}$ times the structural relaxation time.

It would be useful to acquire data at higher temperatures (right side of the plot) but this is not possible with the current apparatus. The cold finger holding the nanocalorimeters is too massive to make the fast temperature jump required to reach the transformation temperature prior to the initiation of transformation. 


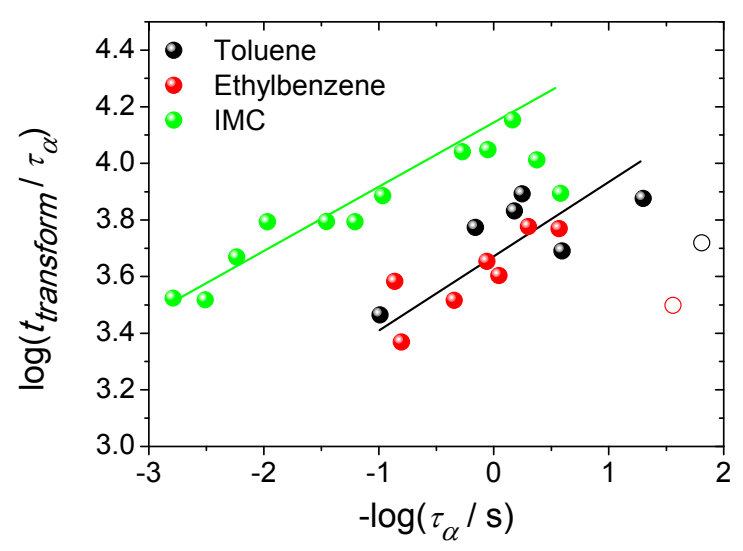

Figure 59: The logarithm of the isothermal transformation time $t_{\text {transform }}$ to the structural relaxation time $\tau_{\alpha}$, as a function of the negative of $\log \left(\tau_{\alpha}\right)$ for three different substances: toluene (black), ethylbenzene (red), and for comparison a high molecular weight glassformer indomethacin (IMC, green). The film thickness of the samples for toluene and ethylbenzene is about $390 \mathrm{~nm}$, the deposition rate is $2 \mathrm{~nm} \mathrm{~s}^{-1}$ and the deposition temperature is $105 \mathrm{~K}$. The IMC data shown is from secondary ion mass spectroscopy (SIMS) measurements on thicker films [136]. The transformation time is calculated for a $390 \mathrm{~nm}$ film by utilizing the observed growth front velocity which was found to be independent of film thickness for films below $1 \mu \mathrm{m}$. The lines are guides to the eye. Open symbols represent data calculated from temperatures scan experiments; see text.

Dawson et al. [137] proposed to estimate the isothermal transformation time from temperature scan experiments to extend the accessible range up to higher temperature. We implemented this procedure to estimate the transformation time, using the onset and ending temperature of the transformation as determined from the measured complex differential temperature amplitude and then dividing by the heating rate. The data points obtained from this approximation are shown in Figure 59 as open symbols. Unfortunately this procedure gave values for toluene and ethylbenzene that are off of the trend line in Figure 59 by more than half an order of magnitude to lower values. Thus the method proposed by Dawson et al. [137] does not seem appropriate for the comparison between $\tau_{\alpha}$ and $t_{\text {transform }}$ here since the uncertainty of temperature determination is too high.

Besides varying the temperature varying the film thickness offers another possibility of investigating stable glass transformation kinetics [138]. We varied the film thickness of stable glasses of toluene from $34 \mathrm{~nm}$ to $2500 \mathrm{~nm}$ and transformed them into the SCL at a certain 
constant temperature slightly above $T_{g}$. The transformation temperature was set in the experiments to meet $-\log \left(\tau_{\alpha}\right)=0$, but usually the uncertainty of temperature determination with the LN2-cooled device yields the transformation temperature to scatter around $-\log \left(\tau_{\alpha}\right)=0$. However, as is observed in Figure 59, $\log \left(\frac{t_{\text {transform }}}{\tau_{\alpha}}\right)$ changes only weakly with temperature and thus even differences in $\log \left(\tau_{\alpha}\right)$ on the order of 1 give negligible differences in $\log \left(\frac{t_{\text {transform }}}{\tau_{\alpha}}\right)$. Nevertheless the differences in the transformation temperature and thus $-\log \left(\tau_{\alpha}\right)$ are corrected to fulfill $-\log \left(\tau_{\alpha}\right)=0$ by assuming the slope of the black line in Figure 59 to be independent of film thicknesses [138].
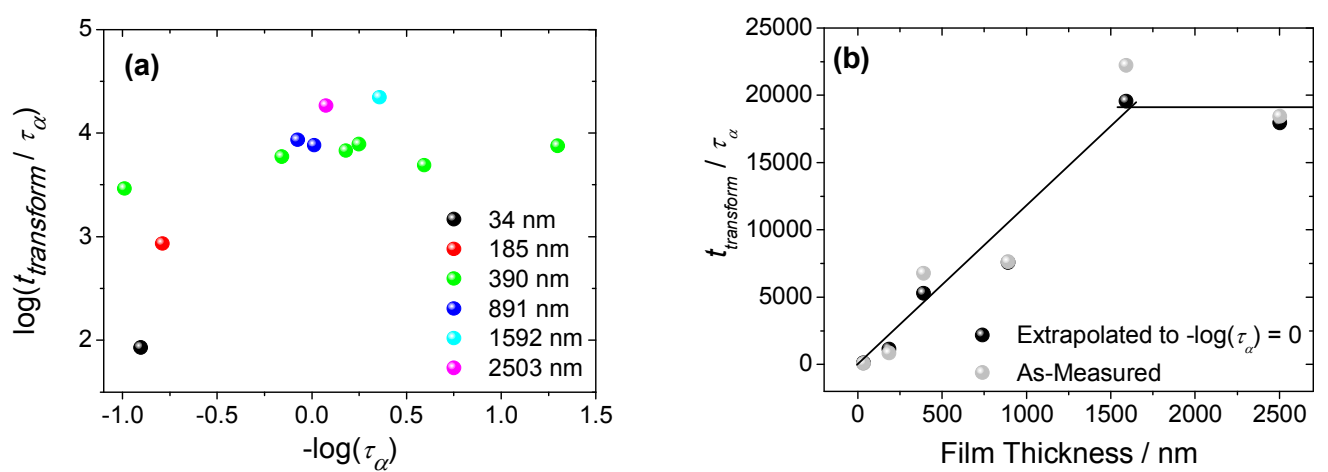

Figure 60: (a) $\log \left(t_{\text {transform }} / \tau_{\alpha}\right)$ over $-\log \left(\tau_{\alpha}\right)$ and (b) $t_{\text {transform }} / \tau_{\alpha}$ over film thickness for stable glasses of toluene. Even though attempts were made to have an uniform transformation temperature across all experiments, the uncertainty of temperature determination results in large differences in $-\log \left(\tau_{\alpha}\right)$. The data of the $390 \mathrm{~nm}$ films (green dots) are taken from Figure 59 and temperature variation is with intent. The grey points in (b) represent the data from (a). The black points in (b) represent the points of (a) extrapolated to match $-\log \left(\tau_{\alpha}\right)=0\left(T_{\text {transform }}=119.45 \mathrm{~K}\right)$. For extrapolation the slope of the black line in Figure 59 was used. If two or more points are available for a certain film thickness the one closest to $-\log \left(\tau_{\alpha}\right)=0$ was used for extrapolation.

Figure 60 (a) shows the logarithm of the thickness dependent transformation time of toluene scaled to $\tau_{\alpha}$ over the negative logarithm of $\tau_{\alpha}$ together with the transformation temperature dependent toluene data of Figure 59. Figure 60 (b) clearly shows the crossover from surface initiated transformation to bulk transformation at a film thickness of about $(1600 \pm 80) \mathrm{nm}$. As determined from the line construction in Figure 60 (b) the transformation time of stable glasses in the thickness independent transformation regime exceeds about $10^{4.2}$ times the structural relaxation time at $-\log \left(\tau_{\alpha}\right)=0$. 


\subsection{Evidence of different liquid states}

As already mentioned in section 4.2, low temperature deposition of ethylbenzene $(\leq 90 \mathrm{~K})$ yield unusually high temperatures of the dynamic glass transition of the first heating scan. This can clearly be seen from Figure 54 (b) and (d). Comparing the phase peaks of the first and second heating scans quantitatively yields a significant temperature difference. Figure 61 shows the result as a function of deposition temperature for ethylbenzene as well as for toluene.

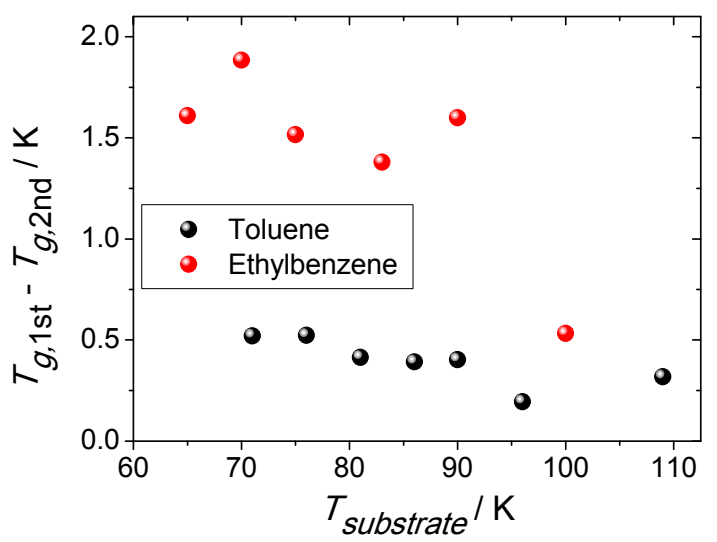

Figure 61: Difference between $T_{g, d y n}$ of toluene and ethylbenzene determined from the first heating of the as-deposited stable glass and from the second heating representing the ordinary glass as a function of deposition temperature $T_{\text {substrate }}$.

The shown data is solely measured with the He-cooled device since a significant deposition temperature dependence cannot be depicted from the data determined with the LN2cooled device down to the lowest accessible deposition temperature of $96 \mathrm{~K}$. As can be seen from Figure 61 significant differences occur for deposition temperatures at and below $90 \mathrm{~K}$. Toluene do not show such a significant difference in $T_{g, d y n}$ between first and second heating. Here the measured difference is comparably small but always positive and scatters around $0.3 \mathrm{~K}$. The difference of $0.3 \mathrm{~K}$ may be due to a systematic error of temperature determination.

The first and second heatings of ethylbenzene yield different $T_{g, d y n}$ of the SCL which gives indication of a liquid-liquid transformation above $T_{g, d y n}$ during the first heating. We expect the transition in the vicinity of $125 \mathrm{~K}$. Consequently, shifting $T_{g, d y n}$ to above $125 \mathrm{~K}$ should result in consistent values for $1^{\text {st }}$ and $2^{\text {nd }}$ heating. For that we have deposited $390 \mathrm{~nm}$ thin films of ethylbenzene at $75 \mathrm{~K}$ and varied the frequency of the measurement from $1 \mathrm{~Hz}$ to $8 \mathrm{kHz}$ which gives a variation in $T_{g, d y n}$ of $7.5 \mathrm{~K}$. 
Figure 62 shows the $1 \mathrm{~Hz}$ (a) and $8 \mathrm{kHz}$ (b) measurements. Phase information at these frequencies is very poor and thus the differences in $T_{g, d y n}$ are determined from the half step height of the differential temperature amplitude. Figure 62 reveals the $1^{\text {st }}$ heating of the $1 \mathrm{~Hz}$ measurement yields a $1.75 \mathrm{~K}$ higher $T_{g, d y n}$ than the ordinary SCL, the $1^{\text {st }}$ heating of the $8 \mathrm{kHz}$ measurement yields still a $0.6 \mathrm{~K}$ higher $T_{g, d y n}$.
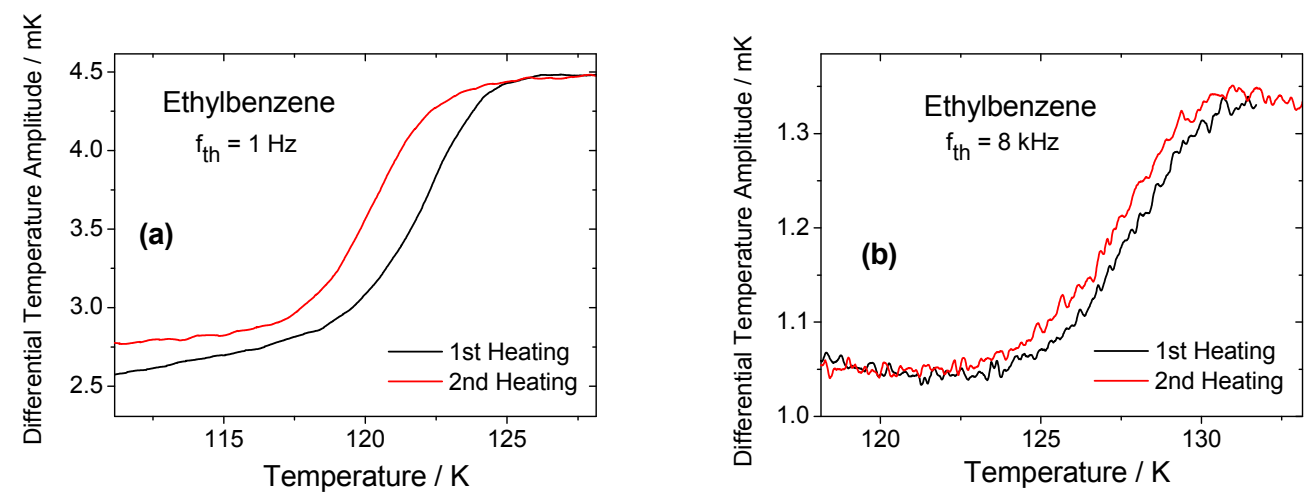

Figure 62: (a) low and (b) high frequency measurement of a $75 \mathrm{~K}$ deposition of ethylbenzene. Shown is the differential temperature amplitude of the first and second heating curves. Heating rate is $0.67 \mathrm{~K} \mathrm{~min}^{-1}$.

High frequencies yield a smaller difference in $T_{g, d y n}$ between the $1^{\text {st }}$ and $2^{\text {nd }}$ heating. The liquid-liquid transformation of the $8 \mathrm{kHz}$ measurement seems to be almost finished prior to the heat capacity increase at the glass transition whereas it has not yet started for the $1 \mathrm{~Hz}$ measurement since the $20 \mathrm{~Hz}$ measurement yields comparable difference in $T_{g, d y n}$. This gives an indication that the liquid-liquid transformation starts in the vicinity of $125 \mathrm{~K}$.

With the information obtained from the frequency dependent investigation of the liquidliquid transformation another interesting experiment is to track the liquid-liquid transformation under quasi-isothermal conditions at temperatures of about $125 \mathrm{~K}$. Unfortunately the current setup of the He-cooled device does not support isothermal transformation experiments on the timescale of a few hours. 


\section{Discussion}

\subsection{Influence of substrate temperature and deposition rate on heat capacity}

While previous studies have shown that vapor-deposited glasses can have lower heat capacities than ordinary glasses, no prior investigation has shown the extent to which deposition rate changes the heat capacity of the glass. Furthermore, in previous work, the only substrate temperatures investigated were $\sim T_{g}$ and $\sim 0.85 T_{g}$. The data reported in Figure 53 to Figure 57 thus provides the first opportunity for a detailed investigation of the impact of substrate temperature and deposition rate on the heat capacity of vapor-deposited glasses.

The heat capacity of organic glasses can have several contributions [37], but in the harmonic approximation we interpret the heat capacity in terms of the vibrational density of states. A lower heat capacity is an indication that the vibrational modes below a few hundred wavenumbers are, on average, shifted to higher frequency. Since the modes associated with intermolecular packing are expected to be in this frequency range, we interpret the lower heat capacities observed here to be indicative of different packing. Previous work [139] has reported that aging a cooled glass for a few days can lower $C_{p}$, but typically by only a small fraction of $1 \%$. In comparison, we observe that vapor-deposited glasses can have $C_{p}$ values as much as $4 \%$ lower than the glass prepared by cooling at $\sim 1 \mathrm{~K} / \mathrm{min}$. The results presented here are consistent with the idea that vapor-deposition can produce "super-aged" glasses with properties that could be achieved by aging an ordinary glass, but only if the aging time exceeded thousands of years [34].

It is of interest to test if the efficient packing that is indicated by the $C_{p}$ measurements is correlated with the kinetic stability of the vapor-deposited glasses. Figure 63 presents such a comparison. This is a cross-plot of two dependent variables, but we choose the $\mathrm{x}$-axis to be the fractional $C_{p}$ decrease, because this is essentially a measure of glass structure. Thus, Figure 63 can be interpreted as a structure-property relationship for vapor-deposited glasses. In this study, we have explored two control variables (deposition rate and substrate temperature). Figure 63 shows data in which substrate temperature is varied at constant deposition rate and in which deposition rate is varied at constant substrate temperature.

Figure 63 was constructed with the idea that a strong correlation would exist between glass structure, expressed by fractional $C_{p}$ decrease, and kinetic stability, as we expect that glasses that are better packed will have lower heat capacities and be stable to higher temperatures when heated. This expectation is reasonably consistent with the data for deposition temperatures 
above $0.8 T_{g}$. The points in Figure 63 which are farthest from the trend line were obtained from depositions below $0.8 T_{g}$.

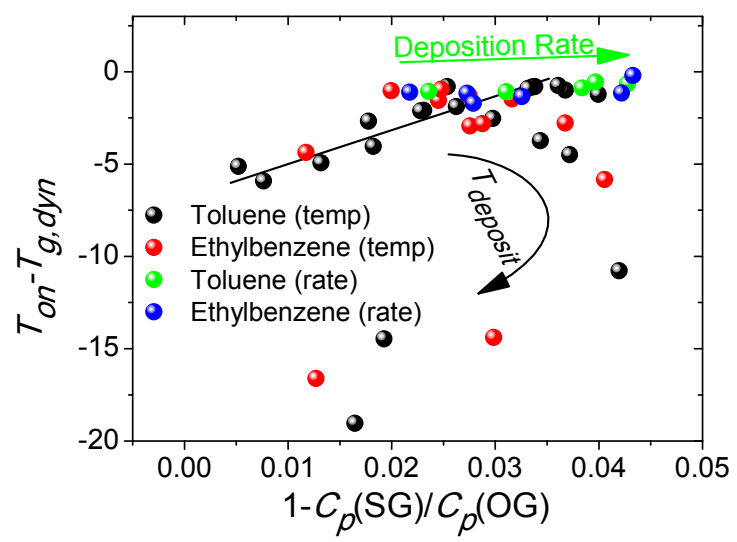

Figure 63: Kinetic stability as a function of fractional $C_{p}$ decrease (as determined from glassy $C_{p}$ of stable and ordinary glass). Black and green points denote the values as determined with toluene, red and blue points the values as determined with ethylbenzene. "Temp" denotes points that are determined by varying the substrate temperature while "rate" denotes points coming from the deposition rate dependence. The black line is a guide to the eye. The black arrow indicates the direction of decreasing deposition temperature. The green arrow indicates the direction of decreasing deposition rate.

If we first restrict our attention to depositions above $0.8 T_{g}$, a reasonably linear correlation is observed between the heat capacity decrease and the kinetic stability. Apparently the relationship between fractional $C_{p}$ decrease and kinetic stability is very similar for vapordeposited glasses of toluene and ethylbenzene. For some of the lowest deposition temperatures, a small step in the heat capacity curve, see Figure 54 (a), here interpreted as $T_{o n}$, occurs below the glass transition temperature. A change in glass properties below $T_{g}$ indicates an unstable glass rather than a stable one. We therefore speculatively identify these very low onset temperatures with unstable glass formation. The formation of unstable glasses at very low deposition temperatures was reported by Hikawa et al. [41] and we expect a change from stable to unstable glass formation by lowering the deposition temperature. Why the heat capacity of the glasses deposited at the lowest temperatures is lower than the ordinary glass is not yet understood. A more detailed study of these effects requires a technique that also allows for characterization of the enthalpy of the deposited glasses, e.g. scanning nanocalorimetry.

A close scrutiny of Figure 63 reveals that the samples prepared by varying the deposition rate may be following a different trend than the samples prepared by varying the substrate 
temperature. It will be very interesting to test whether the same relationship shown in Figure 63 works for other molecules.

\subsection{Effect of deposition temperature on stability}

When a molecule from the vapor phase hits the cold surface of the sample, it becomes part of a mobile surface layer if the temperature is not too low. Depending upon the deposition rates and the mobility of the surface layer, molecules near the surface may have the opportunity to sample multiple configurations and thus the packing moves towards equilibrium [8]. Eventually further deposition moves any particular molecule far enough from the mobile free surface that no further rearrangements are possible. For decreasing deposition temperatures, the driving force for rearrangements towards lower energy levels is increasing but the structural relaxation time in the surface layer is also increasing. These two factors influence the formation of stable glasses in opposite directions. For several substances, the maximum in stability is reported for deposition near $0.85 T_{g}[5,7,8,46]$.

We have observed that the most stable glasses of toluene are formed in a rather broad range of substrate temperatures between $0.8 T_{g}$ to $0.92 T_{g}$ when deposited with a deposition rate of $2 \mathrm{~nm} \mathrm{~s}^{-1}$. For ethylbenzene this range is even broader $\left(0.72 T_{g}\right.$ to $\left.0.96 T_{g}\right)$. Nanocalorimetry data on vapor-deposited glasses of toluene and ethylbenzene were previously presented by LeonGutierrez et al. [46]. These fast scanning measurements show onset temperatures that increase with decreasing substrate temperature and then plateau between about $0.77 T_{g}$ and $0.9 T_{g}$ for toluene and between $0.78 T_{g}$ and $0.96 T_{g}$ for ethylbenzene. While qualitatively similar, in detail the plateau for toluene is shifted to higher substrate temperatures for the work reported here. Although the two studies used very different temperature scanning rates, this difference in optimal substrate temperatures is unexpected.

The first study of the influence of deposition temperature on glass stability [5] gave the impression that IMC and TNB exhibit a narrow range of substrate temperatures where the vapordeposited glasses show the highest kinetic stability. But taking into account the uncertainty of the data in that work [5], a range of deposition temperatures for the most kinetically stable glasses could also describe the IMC and TNB data, with the range encompassing $0.76 T_{g}$ to $0.94 T_{g}$; for this data, the deposition rate was $15 \mathrm{~nm} \mathrm{~s}^{-1}$. Higher precision data, acquired by ellipsometry, shows that vapor-deposition of IMC at $0.2 \mathrm{~nm} \mathrm{~s}^{-1}$ yields glasses of almost identical stability across the range $0.76 T_{g}$ to $0.90 T_{g}$ [10]. A recent theory of Wolynes [135] gives one possible explanation for the broad range of substrate temperatures that produce vapor-deposited glasses of comparable 
kinetic stability for IMC, toluene, and ethylbenzene. The theory, based upon the random first order theory of glass formation, indicates that stable glasses will transform into supercooled liquids via propagating growth fronts. For sufficiently stable glasses, the front velocity is predicted to reach a limiting value that depends only on the supercooled liquid mobility. From this perspective, it is reasonable that thin stable glass films (such that transformation is entirely controlled by the growth front velocity) might exhibit a broad plateau in the onset temperature and other measures of kinetic stability.

An interesting comparison can also be made with recent adiabatic calorimetry measurements of vapor-deposited glasses of ethylbenzene by Ramos et al. [7]. They observed a quite sharp maximum in kinetic stability at $0.9 T_{g}$ and a quite sharp minimum in the enthalpy at the same temperature. We can speculate on why our measurements of the kinetic stability of ethylbenzene glasses show a plateau region in terms of deposition temperature while Ramos et al. observe a sharp peak. Even though the deposition rate used by Ramos et al. [7] is comparable to the value used here $\left(2 \mathrm{~nm} \mathrm{~s}^{-1}\right)$, their sample thickness is orders of magnitude larger. For thick films, the theory of Wolynes predicts that the kinetic stability always depends to some extent upon the enthalpy of the glass [135]. As discussed above, this differs from predictions for the thin film regime. Thus, within this theory, it is possible for the kinetic stability of otherwise identical vapordeposited glasses to show different trends as a function of substrate temperature; thin films could show a plateau temperature regime while thick films show a more pronounced maximum. Physically, these theoretical results can be understood as follows [138]. Thin films completely transform into the supercooled liquid by a constant velocity growth front with no induction time; in this regime, the growth front velocity completely controls kinetic stability. Thicker films (greater than $\sim 1 \mu \mathrm{m}$ ) also transform within the bulk with transformation beginning from "nucleation sites." As lower energy glasses are prepared, it is likely that the density of these sites continuously decreases and this would cause a steady increase in kinetic stability for thick films that need not be observed for thin films. Aspects of this scenario are consistent with previously published experimental results $[51,138]$ but further experiments are required to fully test these ideas.

\subsection{Effect of deposition rate on stability}

As the deposition rate is lowered, molecules have more time to sample packing arrangements with their neighbors before they are buried by other molecules coming from the vapor. In this simple view, slower deposition should yield glasses that are lower on the energy 
landscape and exhibit higher kinetic stability. Below some limiting deposition rate, further changes in rate should not influence the kinetic stability of the resulting glass. This could occur either because the surface layer is fully equilibrated or because the thin glassy film has become sufficiently stable that the growth front velocity does not change with further decreases in the enthalpy.

As shown in Figure 57, the deposition rate has a very small influence on the kinetic stability of vapor-deposited glasses of ethylbenzene and toluene. As described above, this result is consistent with studies by Leon-Gutierrez et al. [46] on toluene and ethylbenzene glasses. In contrast, over nearly the same range of deposition rates, IMC and TNB show a 10 to $15 \mathrm{~K}$ increase in the onset temperature [34]. This may be due to the different film thicknesses of the IMC and TNB samples $(\sim 20 \mu \mathrm{m})$ versus $\sim 400 \mathrm{~nm}$ for toluene and ethylbenzene. As described above, thicker films transform via "nucleation sites" that introduce a complexity not found in thin film samples.

Alternately, the small influence of deposition rate on kinetic stability could be explained by assuming that our glasses of ethylbenzene and toluene are nearly fully equilibrated for deposition at $105 \mathrm{~K}$ and thus deposition rate has little impact on the properties of the resulting glasses. This is in agreement with Ramos et al. who reported that glasses of ethylbenzene vapordeposited at $105 \mathrm{~K}$ have the enthalpy expected for the supercooled liquid at this temperature; this result would indicate that deposition rates slower than $2 \mathrm{~nm} \mathrm{~s}^{-1}$ would not be expected to yield further property changes. On the other hand, Leon-Gutierrez et al. [46] report that deposition of ethylbenzene and toluene at $105 \mathrm{~K}$ at rates between 0.001 and $0.1 \mathrm{~nm} \mathrm{~s}^{-1}$ yield glasses that have considerably higher enthalpy values than expected at equilibrium. Since we do not expect that lower deposition rates should lead to higher enthalpy values, we know of no scenario that reconciles the enthalpy data from refs. [46] and [7]. To investigate the importance of potential equilibration on the deposition rate dependence, it would be useful to perform similar experiments at a lower substrate temperature.

If the glasses deposited here at $105 \mathrm{~K}$ are not fully equilibrated, then it is difficult to understand why the deposition rate has such a small impact on glass properties. One scenario that could reconcile a weak deposition rate dependence with a non-equilibrium structure is the following. Recent work has shown that stable glasses prepared by vapor deposition can be anisotropic [10, 137]. This suggests that surface mobility can lead to partially equilibrated structures that are influenced by the presence of the nearby interface (and thus are not completely representative of equilibrated structures in the bulk). For toluene and ethylbenzene, it could be that such surface equilibrated structures are readily obtained near $105 \mathrm{~K}$, thus accounting for the weak deposition rate dependence. Using the energy landscape picture: some molecules (or 
clusters of molecules) are trapped in a number of neighboring metastable states close to equilibrium. Even if the deposition rate is lowered further some of them still cannot get into the equilibrium state due to barrier separation.

\subsection{Transformation time vs. structural relaxation time}

As described previously, when thin films of stable glasses are heated above $T_{g}$ they transform into supercooled liquids via a surface-initiated growth front $[135,140]$.

Molecules in the center of a thin film are so tightly packed that they become "unjammed" by waiting for mobility to arrive from a distance. The growth front starts at the free surface since the mobile surface layer is already in the supercooled liquid [49]. The growth front has been observed to propagate into the stable glass at constant velocity. The linear transformation behavior shown in Figure 58 is consistent with a constant velocity growth front.

Figure 59 shows remarkable similarity in the transformation properties of stable glasses of toluene, ethylbenzene and IMC when the annealing temperature is replaced by the structural relaxation time $\tau_{\alpha}$ of the supercooled liquid at that temperature. All measurements shown in Figure 59 correspond to a film thickness of about $400 \mathrm{~nm}$. Consistent with a recent analysis by Wolynes [135], the trend curve of Figure 59 indicates that the mobility of the supercooled liquid controls the rate at which a growth front can move into the stable glass.

As shown in Figure 59, the transformation time $t_{\text {transform }}$ has a weaker dependence on temperature than the structural relaxation time $\tau_{\alpha}$ does. This is also the case for three isomers of tris-naphthylbenzene (TNB) [137]. A change in the structural relaxation time by 2 orders of magnitude causes a change in the ratio $\frac{t_{\text {transform }}}{\tau_{\alpha}}$ by 0.5 orders of magnitude. Wolynes has suggested that the different temperature dependences of $t_{\text {transform }}$ and $\tau_{\alpha}$ might be related to spatially heterogeneous dynamics in the supercooled liquid [135]. He draws the picture of a glass consisting of a "fluctuating mosaic of structures that locally are chosen from a diverse set of minimum-energy patterns that reconfigure through activated events." During transformation of the stable glass to the SCL activated transitions (that originate mobility) of small regions in the stable glass are "driven by the extensive configurational entropy of local structural patterns. Such transitions (sometimes called "entropic droplets") are retarded by the stability of the local pattern, which varies spatially, giving a distribution of relaxation times." However, reconfigurational activation free energy is reduced at the free surface by a factor of 2 and thus mobility is generated much more rapidly at the free surface. Anisotropy in the bulk and spatial variation of local patterns in the mobile surface layer cause spatial distributions of mobility generation in such a 
heterogeneous rejuvenation. On average though the rejuvenation proceeds by front propagation with constant velocity.

Besides the temperature dependence, the transformation time was investigated regarding its dependence on film thickness. As can be seen from Figure 60 as long as the investigated film thickness is below $(1600 \pm 80) \mathrm{nm}$ the transformation time is thickness dependent, above it becomes thickness independent. Such a characteristic thickness dependence was also found by Kearns et al. [51] for IMC by AC-nanocalorimetry and quasi-isothermal TMDSC where the regime of constant velocity growth front applied to films thinner than $1000 \mathrm{~nm}$. This behavior is consistent with the picture of two different competitive heterogeneous transformation mechanisms, a surface-initiated and a bulk transformation mechanism. For thin films, the heterogeneous mechanism described by a surface initiated growth front is the major mechanism of transformation. Stevenson and Wolynes [141] have shown that random first order theory implies a reduction of the reconfigurational activation free energy by a factor of 2 at a free mobile surface in an equilibrated liquid. Thus mobility will proceed much faster from the mobile layer into the bulk by front propagation.

Bulk transformation originates at activated reconfiguration events that start to increase the mobility in neighboring regions. It is assumed that structural defects in the bulk can initiate growth fronts into the surrounding of the stable glass. The mobility increase is "autocatalytic and therefore a front of higher fictive temperature should emanate radially from each initially rejuvenated center. These growing zones of influence will quickly overlap and the glass, as a whole, will rapidly be equilibrated" [135].

Surface-initiated transformation proceeds with constant velocity, bulk transformation proceeds accelerated (but at constant growth front velocity), if not slowed down when growing regions of the SCL impinge. From that it can easily be realized that after a certain crossover transformation time (depending on transformation temperature) bulk transformation takes over the major role in transformation. If the film thickness is small enough to obtain transformation times below the crossover transformation time surface-initiated transformation is primary.

\subsection{Influence of deposition temperature on the liquid state}

It is known that dynamics in SCLs are spatially heterogeneous. "In a supercooled liquid, spontaneous fluctuations cause different regions of the sample to transiently experience different relaxation times; regions only a few nanometers apart can relax at rates that differ by more than a factor of 100" [49] (see further [142, 143]). However, Singh et al. [144] did simulations that show 
stable glasses have a much more homogeneous structure than ordinary glasses since the average cluster size is smaller. The question is if this higher homogeneity would have an influence on the SCL state obtained from transforming the stable glass.

Recently, Ishii et al. [145] investigated vapor-deposited alkylbenzenes by light scattering [146] and found high density glasses (HDG) for deposition at about $0.9 T_{g}$ and low density glasses (LDG) for deposition much lower than $T_{g}$. Further heating of the glasses yielded high density (HDL) and low density liquids (LDL), respectively. Moreover, Ishii et al. found the LDL to transform into the HDL at elevated temperatures. This indicates that a stable glass may transform into a different liquid than a slowly cooled glass and thus is an example of polyliquidism. While polyamorphism has been reported for several materials [147-152] there are only a few examples for polyliquidism (e.g. [153]). Further, the observed relaxation of the liquid formed from a stable glass towards the ordinary SCL can be regarded as a liquid-liquid transition.

AC calorimetry allows a measurement of molecular mobility. By using a thermal frequency of $20 \mathrm{~Hz}$ the molecules of the SCL at $T_{g, d y n}(20 \mathrm{~Hz})$ rearrange on the timescale of the oscillation at about $1 /(2 \pi f)=8 \cdot 10^{-3} \mathrm{~s}$. If a SCL is unusually mobile, as it is in the observations of Ishii, the same rearrangement occurs at a lower temperature and thus $T_{g, d y n}$ is reduced.

AC nanocalorimetry on vapor-deposited glasses of toluene and ethylbenzene leads to no detectable change in the glass transition temperature if deposited in the vicinity of $0.85 T_{g}$. However, we see a shift in the glass transition for ethylbenzene to higher temperatures if deposited at and below $0.78 T_{g}$, i.e. glasses of low kinetic stability. This feature is not an artifact of the measurement since it is not seen for toluene deposited and measured under exactly the same conditions. Moreover, the new state of SCL seems to transform to the ordinary SCL and $T_{g, d y n}$ retains the normal value. Thus we see evidence of different states of SCLs for ethylbenzene. The mobility of the resulting liquid from a vapor-deposited glass at and below $0.78 T_{g}$ is lower as compared to an ordinary SCL which is in agreement with observation by Capponi et al. for supercooled polyols grown by vapor-deposition.

We have varied the applied frequency to vary $T_{g, d y n}$ between $T_{g}+5 \mathrm{~K}$ to $T_{g}+12 \mathrm{~K}$ to gain information about the stability of the new liquid state. Within this temperature range the structural relaxation time of ethylbenzene varies by about 5 orders of magnitude. Figure 62 shows that the transformation speeds up with increasing temperature but transformation time remains much longer than the structural relaxation time which is on the order of milliseconds at $T_{g}+12 \mathrm{~K}$. Why ethylbenzene deposited at relatively low temperature yields highly stable liquid states may be connected with the structure formed during deposition. Singh et al. [144] found the configurational entropy of vapor-deposited glasses to increase below the optimal deposition 
temperatures. In this respect, with lower deposition temperature the structure of the vapordeposited glasses develops into more and more inhomogeneous structures. In this respect we speculate low temperature deposited glasses to show extensive cluster formation. Resulting spatial inhomogeneities in the liquid finally raise the average relaxation time.

On the other hand toluene shows neither enhanced mobility nor reduced mobility in the liquid state even if vapor-deposited in a large range of deposition temperatures. This indicates that the formation of new liquids by transformation of vapor-deposited glasses depends on the material (or at least a certain material property) or that the stability of a possible less mobile liquid causes the liquid-liquid transformation to already be finished at the onset of the $20 \mathrm{~Hz}$ glass transition.

It would be interesting to investigate the transformation between two different liquid states at quasi-isothermal conditions by $\mathrm{AC}$ nanocalorimetry. Equivalent to the investigations regarding the stable glass-to-SCL transformation, these transformation times can also be related to the structural relaxation time. Unfortunately the current setup of the He-cooled device does not allow for reliable measurements on this issue but the experimental setup will enable this kind of measurement soon. 


\section{Conclusion}

The differential AC chip calorimeter in combination with a physical vapor deposition setup was successfully applied for deposition and in-situ heat capacity measurements of thin films of highly volatile materials. The expected features of as-deposited stable glasses can be measured with high accuracy, e.g., the onset temperature of the stable glass to supercooled liquid transition on heating or the ratio of the heat capacity for the as-deposited and the ordinary glasses. The main advantage of the AC calorimeter compared to scanning calorimetry is the capability to perform quasi-isothermal measurements and to follow the stable glass to supercooled liquid transformation process with time.

A finite element model is established that quantitatively describes the chip calorimeter used here and allows the calculation of the film thickness if specific heat capacity is known for at least one temperature. The FEM analysis provides detailed insight into the complex relationship between heat capacity (or film thickness) and the measured differential temperature amplitude. The measured differential temperature amplitude, while not strictly proportional to the sample heat capacity, provides a useful estimate of sample heat capacity as a function of temperature.

To test the sensitivity of the constructed device we have lowered the film thickness to the limit. Heat capacity measurements were able to be performed for thin films of toluene down to $2 \mathrm{~nm}$. Different from other observations $[61,134]$ on membrane suspended thin films we see no $T_{g}$ dependence on film thickness. Nevertheless this is consistent with earlier thin film measurements on PS and PMMA with former versions of the chip-nanocalorimeters used in this work [89].

We have investigated the properties of vapor-deposited glasses of toluene and ethylbenzene, varying both the substrate temperature and deposition rate across a significant range. Consistent with previous work, we observed that glasses deposited near $0.85 T_{g}$ have lower heat capacity and high kinetic stability. During isothermal transformation into the supercooled liquid, such glasses exhibited linear kinetics consistent with a surface-initiated, constant velocity growth front. For films of toluene thicker than $1600 \mathrm{~nm}$ a nonlinear transformation incurs the major role of transformation. The transformation times for toluene and ethylbenzene stable glasses, relative to the structural relaxation times of the supercooled liquids, are similar to those obtained previously for indomethacin, indicating that glasses of comparable kinetic stability are being formed for all three systems. If the substrate temperature is held at temperatures in the vicinity of 
$T_{g}$, vapor-deposited glasses of toluene and ethylbenzene are nearly indistinguishable from liquidcooled glasses.

The effect of the deposition rate on the properties of vapor-deposited toluene and ethylbenzene glasses, across a range of more than 3 orders of magnitude, was surprisingly modest. Previous work on IMC and TNB (higher $T_{g}$ systems) reported significantly larger effects of deposition rate on kinetic stability. As discussed above, it is possible that this difference is due to the much thicker films used in the IMC and TNB experiments and the role of bulk "nucleation" of the supercooled liquid in thick films. In future work, it would be useful to compare the impact of deposition rate at a given temperature in the thin and thick film regimes.

The present work provided the first opportunity to examine the correlation between a significant heat capacity decrease and high kinetic stability. For substrate temperatures above $0.8 T_{g}$, these quantities show a reasonable correlation whether the substrate temperature or the deposition rate are varied. At lower substrate temperatures, kinetic stability is lost while the heat capacity remains lower than in the ordinary glass. This intriguing observation deserves further attention.

Low temperature deposition of ethylbenzene yielded unexpected low mobility of the resulting supercooled liquid. While transformed stable glasses deposited above $0.78 T_{g}$ show usual dynamic glass transition temperatures, SCLs obtained from vapor-deposition below $0.78 T_{g}$ show a remarkably higher $T_{g, d y n}$. Interestingly this feature is not observed for toluene. The stability of such less mobile SCL was qualitatively investigated by temperature ramping and yielded that the SCL still exhibits lower mobility even if the temperature was ramped up to $T_{g}+12 \mathrm{~K}$. 


\section{Outlook}

Some aspects regarding film thickness and heat capacity determination are still under development. Film thickness is determined by FEM so far, but some other approaches to obtain the film thickness independently from a FEM are desirable since changes of the sensor by the manufacturer necessitates new modeling of the membrane each time. Heat capacity cannot be accessed even with the help of the FEM since we cannot precisely estimate the radius or amount of the material contributing to the differential temperature amplitude. Using masks to deposit samples with defined dimensions would be useful. Unfortunately the sensors used here do not allow masking.

The results of the performed series of experiments revealed some deficiencies regarding the chosen experimental parameters, leaving room for improvements. The deposition rate dependence, for example, yielded only a very weak effect on stability even though from other works a larger effect was expected. This may be a result of the relatively high deposition temperature at the upper bound of the stability plateau, yielding nearly equilibrated vapordeposited glasses. It would be interesting to see if lower deposition temperatures give a larger rate dependence.

Very recently we have observed indications of a different state of SCL of ethylbenzene. This feature is worth investigating more extensively regarding its formation process and transformation kinetics towards the ordinary SCL. Moreover further investigation of a possible mobile/immobile SCL formation for toluene is needed to clarify whether toluene also forms an extraordinary SCL or not. The He-cooled device will be equipped to enable such investigations. The planned combination of the device with fast scanning calorimetry is a useful approach to investigate less stable extraordinary SCLs.

An issue still to solve is the impact of stress and strain on the measured quantities. We know that the heater resistance reacts very sensitively to stress on the membrane. However, appropriate handling of the heater resistance data allows the exclusion of stress affected data. This is necessary for reliable temperature determination with the LN2-cooled device. Another quantity we have to take care of regarding stress and strain is the heat capacity and thus the differential temperature amplitude. Lion et al. [131] found the measured heat capacity across the glass transition to be dependent on the differences in the thermal expansion coefficients of the substrate and the sample. The investigations of Lion et al. were geared towards fast scanning calorimetry and the heating rates we used are far below what was considered. Also, Lion et al. investigated 
liquid-cooled glasses that have smaller moduli as compared to the stable vapor-deposited glasses, which exhibit moduli about 15\% higher than their corresponding ordinary glasses [55]. Moreover, as the temperature difference between the dynamic glass transition temperature and the deposition temperature increases, the effect coming from the different thermal expansion coefficients of the sample and the membrane will not be negligible. This also influences the measured heat capacity. The lower the deposition temperature the bigger the effect will be. So far we do not see any indication of a stress effect in the differential temperature amplitude at the dynamic glass transition, neither for the most stable vapor-deposited glasses nor for glasses deposited at very low temperatures. 
[1] M. D. Ediger and P. Harrowell, Perspective: Supercooled Liquids and Glasses. J. Chem. Phys., 2012. 137(8): p. 080901.

[2] C. A. Angell, K. L. Ngai, et al., Relaxation in Glassforming Liquids and Amorphous Solids. J. Appl. Phys., 2000. 88(6): p. 3113-3157.

[3] W. Klement, R. H. Willens, and P. Duwez, Non-Crystalline Structure in Solidified Gold-Silicon Alloys. Nature, 1960. 187(4740): p. 869-870.

[4] G. Tammann, Der Glaszustand. 1933, Leipzig: Leopold Voss.

[5] K. L. Kearns, S. F. Swallen, et al., Influence of Substrate Temperature on the Stability of Glasses Prepared by Vapor Deposition. J. Chem. Phys., 2007. 127(15): p. 154702.

[6] K. Ishii, H. Nakayama, et al., Anomalously high-density glass of ethylbenzene prepared by vapor deposition at temperatures close to the glass-transition temperature. Chem. Phys. Lett., 2008. 459(1-6): p. 109-112.

[7] S. L. L. M. Ramos, M. Oguni, et al., Character of Devitrification, Viewed from Enthalpic Paths, of the Vapor-Deposited Ethylbenzene Glasses. J. Phys. Chem. B, 2011. 115(49): p. 1432714332.

[8] S. F. Swallen, K. L. Kearns, et al., Organic Glasses with Exceptional Thermodynamic and Kinetic Stability. Science, 2007. 315(5810): p. 353-356.

[9] E. Leon-Gutierrez, A. Sepúlveda, et al., Stability of thin film glasses of toluene and ethylbenzene formed by vapor deposition: An in situ nanocalorimetric study. Phys. Chem. Chem. Phys., 2010. 12: p. 14693-14698.

[10] S. S. Dalal, A. Sepúlveda, et al., Density and Birefringence of a Highly Stable alpha, alpha, beta-Trisnaphthylbenzene Glass. J. Chem. Phys., 2012. 136(20): p. 204501.

[11] H. Huth, A. Minakov, and C. Schick, High Sensitive Differential AC-Chip Calorimeter for Nanogram Samples. Netsu Sokutei, 2005. 32(2): p. 70-76.

[12] H. Huth, A. A. Minakov, and C. Schick, Differential AC-Chip Calorimeter for Glass Transition Measurements in Ultrathin Films. J. Polym. Sci. B Polym. Phys., 2006. 44(20): p. 2996-3005.

[13] M. Ahrenberg, E. Shoifet, et al., Differential Alternating Current Chip Calorimeter for In-situ Investigation of Vapor-deposited Thin Films. Rev. Sci. Instrum., 2012. 83(3): p. 033902.

[14] H. Vogel, Das Temperaturabhängigkeitsgesetz der Viskosität. Phys. Z., 1921. 22: p. 645-646.

[15] G. S. Fulcher, Analysis of recent Measurements of the Viscosity of Glasses. J. Am. Ceram. Soc., 1925. 8(6): p. 339-355.

[16] P. G. Debenedetti and F. H. Stillinger, Supercooled Liquids and the Glass Transition. Nature, 2001. 410(6825): p. 259-267.

[17] H. R. Lillie and H. N. Ritland, Fine Annealing of Optical Glass. J. Am. Ceram. Soc., 1954. 37(10): p. 466-473.

[18] C. T. Moynihan, A. J. Easteal, and J. Wilder, Dependence of the Glass Transition Temperature on Heating and Cooling Rate. J. Phys. Chem., 1974. 78(26): p. 2673-2677.

[19] I. M. Hodge, Enthalpy Relaxation and Recovery in Amorphous Materials [Review]. J. NonCryst. Solids, 1994. 169(3): p. 211-266.

[20] A. Q. Tool, Relation between Inelastic Deformability and Thermal Expansion of Glass in its Annealing Range. J. Am. Ceram. Soc., 1946. 29(9): p. 240-253.

[21] A. Q. Tool and C. G. Eichlin, Variations Caused in the Heating Curves of Glass by Heat Treatment. J. Am. Ceramic Soc., 1931. 14(4): p. 276-308.

[22] P. Lunkenheimer and A. Loidl, Dielectric Spectroscopy of Glass-Forming Materials: alphaRelaxation and Excess Wing. Chem. Phys., 2002. 284(1-2): p. 205-219.

[23] S. Weyer, M. Merzlyakov, and C. Schick, Application of an Extended Tool-NarayanaswamyMoynihan Model Part 1. Description of Vitrification and Complex Heat Capacity measured by Temperature-Modulated DSC. Thermochim. Acta, 2001. 377(1-2): p. 85-96. 
[24] S. Weyer, Beschreibung des Einfrierens und der komplexen Wärmekapazität am Glasübergang mit einem erweiterten TNM-Modell. 2002: Rostock. p. 1-167.

[25] C. A. Angell, Strong and Fragile Liquids. in: Relaxation in complex systems ed. by K. L. Ngai and G. B. Wright (NTIS, Springfield, Va.), 1984: p. 3-11.

[26] R. Bohmer, K. L. Ngai, et al., Nonexponential Relaxations in Strong and Fragile Glass Formers. J. Chem. Phys. , 1993. 99(5): p. 4201-4209.

[27] A. Loidl, H. S. L., et al., Relaxational Dynamics at the Glass Transition in Orientational and Canonical Glasses Investigated by Dielectric Spectroscopy. J. Mol. Liq., 1991. 49(2): p. 133139.

[28] S. L. Simon, D. J. Plazek, et al., Physical Aging of a Polyetherimide - Volume Recovery and Its Comparison to Creep and Enthalpy Measurements. J. Polym. Sci., Part B: Polym. Phys., 1997. 35(6): p. 929-936.

[29] P. W. Anderson, B. I. Halperin, and C. M. Varma, Anomalous low-temperature thermal properties of glasses and spin glasses. Philos. Mag., 1972. 25(1): p. 1-9.

[30] J. D. Stevenson and P. G. Wolynes, Thermodynamic - Kinetic correlations in supercooled liquids: A critical survey of experimental data and predictions of the random first-order transition theory of glasses. J. Phys. Chem. B, 2005. 109(31): p. 15093-15097.

[31] L. M. Wang, C. A. Angell, and R. Richert, Fragility and Thermodynamics in Nonpolymeric Glass-Forming Liquids. J. Chem. Phys., 2006. 125(7): p. 074505.

[32] W. Kauzmann, The Nature of the Glassy State and the Behavior of Liquids at Low Temperatures. Chem. Rev., 1948. 43(2): p. 219-256.

[33] M. Wolfgardt, J. Baschnagel, et al., Entropy of glassy polymer melts: Comparison between Gibbs-DiMarzio theory and simulation. Phys. Rev. E, 1996. 54(2): p. 1535-1543.

[34] K. L. Kearns, S. F. Swallen, et al., Hiking Down the Energy Landscape: Progress Towards the Kauzmann Temperature via Vapor Deposition. J. Phys. Chem. B, 2008. 112(16): p. 4934-4942.

[35] F. H. Stillinger and T. A. Weber, Hidden structure in liquids. Physical Review A, 1982. 25(2): p. 978-989.

[36] A. Heuer, Exploring the Potential Energy Landscape of Glass-Forming Systems: from Inherent Structures via Metabasins to Macroscopic Transport. J. Phys.-Condens. Mat., 2008. 20(37): p. 373101.

[37] M. Goldstein, Viscous Liquids and the Glass Transition: A Potential Energy Barrier Picture. J. Chem. Phys. , 1969. 51(9): p. 3728-3739.

[38] C. Schick, Chapter 16 Temperature modulated differential scanning calorimetry (TMDSC)basics and applications to polymers, in Handbook of Thermal Analysis and Calorimetry, S. Z. D. Cheng, Editor. 2002, Elsevier Science B.V. p. 713-810.

[39] M. Sugisaki, H. Suga, and S. Seki, Calorimetric Study of the Glassy State. 3. Novel Type Calorimeter for Study of Glassy State and Heat Capacity of Glassy Methanol. B. Chem. Soc. Jpn., 1968. 41(11): p. 2586-2591.

[40] O. Haida, H. Suga, and S. Seki, Realization of the Glassy State of some simple Liquds by the Vapor Condensation Method. Thermochim. Acta, 1972. 3(3): p. 177-180.

[41] H. Hikawa, M. Oguni, and H. Suga, Construction of an Adiabatic Calorimeter for a VaporDeposited Sample and Thermal Characterization of Amorphous Butyronitrile. J. Non-Cryst. Solids, 1988. 101(1): p. 90-100.

[42] K. Takeda, O. Yamamuro, and H. Suga, Calorimetric Study on Structural Relaxation of 1Pentene in Vapor-Deposited and Liquid-Quenched Glassy States. J. Phys. Chem., 1995. 99(5): p. 1602-1607.

[43] M. K. Mapes, S. F. Swallen, et al., Isothermal Desorption Measurements of Self-Diffusion in Supercooled o-Terphenyl. J. Chem. Phys., 2006. 124(5): p. 054710.

[44] K. J. Dawson, K. L. Kearns, et al., Highly Stable Indomethacin Glasses Resist Uptake of Water Vapor. J. Phys. Chem. B, 2009. 113(8): p. 2422-2427.

[45] K. L. Kearns, S. F. Swallen, et al., Calorimetric Evidence for two Distinct Molecular Packing Arrangements in Stable Glasses of Indomethacin. J. Phys. Chem., 2009. 113(6): p. 1579-1586. 
[46] E. Leon-Gutierrez, A. Sepúlveda, et al., Stability of Thin Film Glasses of Toluene and Ethylbenzene Formed by Vapor Deposition: An In situ Nanocalorimetric Study. Phys. Chem. Chem. Phys., 2010. 12(44): p. 14693-14698.

[47] K. Ishii, H. Nakayama, and R. Moriyama, Nonequilibrium and Relaxation in Deeply Supercooled Liquid of Isopropylbenzene Obtained through Glass Transition from VaporDeposited Glass. J. Phys. Chem. B, 2012. 116(3): p. 935.

[48] L. Zhu and L. Yu, Generality of Forming Stable Organic Glasses by Vapor Deposition. Chem. Phys. Lett., 2010. 499(1-3): p. 62-65.

[49] S. F. Swallen, K. Traynor, et al., Stable Glass Transformation to Supercooled Liquid via Surfaceinitiated Growth Front. Phys. Rev. Lett., 2009. 102(6): p. 065503.

[50] E. León-Gutierrez, G. Garcia, et al., Glass Transition in Vapor Deposited Thin Films of Toluene. Thermochim. Acta, 2009. 492(1-2): p. 51-54.

[51] K. L. Kearns, K. R. Whitaker, et al., Observation of low Heat Capacities for Vapor-Deposited Glasses of Indomethacin as determined by AC Nanocalorimetry. J. Chem. Phys., 2010. 133(1): p. 014702.

[52] E. Leon-Gutierrez, G. Garcia, et al., Size Effects and Extraordinary Stability of Ultrathin Vapor Deposited Glassy Films of Toluene. J. Phys. Chem. Lett., 2010. 1(1): p. 341-345.

[53] K. R. Whitaker, Ahrenberg, M., Schick, C., Ediger, M.D., Vapor-deposited alpha, alpha, betaTris-Naphthylbenzene Glasses with Low Heat Capacity and High Kinetic Stability. J. Chem. Phys., 2012. 137(15): p. 154502.

[54] Z. Fakhraai, T. Still, et al., Structural Variations of an Organic Glassformer Vapor-Deposited onto a Temperature Gradient Stage. J. Phys. Chem. Lett., 2011. 2(5): p. 423-427.

[55] K. L. Kearns, T. Still, et al., High-Modulus Organic Glasses Prepared by Physical Vapor Deposition. Adv. Mater., 2010. 22(1): p. 39-42.

[56] K. J. Dawson, L. Zhu, et al., Anisotropic Structure and Transformation Kinetics of VaporDeposited Indomethacin Glasses. J. Phys. Chem. B, 2011. 115(3): p. 455-463.

[57] R. C. Bell, H. F. Wang, et al., Nanometer-resolved Interfacial Fluidity. J. Am. Chem. Soc., 2003. 125(17): p. 5176-5185.

[58] C. J. Ellison and J. M. Torkelson, The Distribution of Glass-Transition Temperatures in Nanoscopically Confined Glass Formers. Nat. Mater., 2003. 2(10): p. 695-700.

[59] S. Kawana and R. A. L. Jones, Character of the Glass Transition in Thin Supported Polymer Films. Phys. Rev. E, 2001. 63(2): p. 021501.

[60] J. L. Keddie, R. A. L. Jones, and R. A. Cory, Interface and Surface Effects on the GlassTransition Temperature in Thin Polymer-Films. Faraday Discuss., 1994. 98: p. 219-230.

[61] J. A. Forrest, K. Dalnoki-Veress, et al., Effect of Free Surfaces on the Glass Transition Temperature of Thin Polymer Films. Phys. Rev. Lett., 1996. 77(10): p. 2002-2005.

[62] S. Kim and J. M. Torkelson, Distribution of Glass Transition Temperatures in Free-Standing, Nanoconfined Polystyrene Films: A Test of de Gennes' Sliding Motion Mechanism. Macromolecules, 2011. 44(11): p. 4546-4553.

[63] T. Kerle, Z. Lin, et al., Mobility of Polymers at the Air/Polymer Interface. Macromolecules, 2001. 34(10): p. 3484-3492.

[64] P. Gasemjit and D. Johannsmann, Thickness of the Soft Layer on Glassy Polystyrene Surfaces. J. Polym. Sci. Pol. Phys., 2006. 44(20): p. 3031-3036.

[65] Z. Fakhraai and J. A. Forrest, Measuring the Surface Dynamics of Glassy Polymers. Science, 2008. 319(5863): p. 600-604.

[66] K. Paeng, H.-N. Lee, et al., Temperature-Ramping Measurement of Dye Reorientation to probe Molecular Motion in Polymer Glasses. J. Chem. Phys., 2011. 134(2): p. 024901.

[67] K. Paeng, R. Richert, and M. D. Ediger, Molecular Mobility in Supported Thin Films of Polystyrene, Poly(methyl methacrylate), and Poly(2-vinyl pyridine) probed by Dye Reorientation. Soft Matter, 2012. 8(3): p. 819-826. 
[68] S. F. Swallen, K. Windsor, et al., Transformation of Stable Glasses into Supercooled Liquids: Growth Fronts and Anomalously Fast Liquid Diffusion. J. Phys. Chem. B, 2010. 114(8): p. 2635-2643.

[69] Y. J. Jung, J. P. Garrahan, and D. Chandler, Excitation Lines and the Breakdown of StokesEinstein Relations in Supercooled Liquids. Phys. Rev. E, 2004. 69(6): p. 061205.

[70] G. H. Fredrickson and H. C. Andersen, Kinetic Ising-Model of the Glass-Transition. Phys. Rev. Lett., 1984. 53(13): p. 1244-1247.

[71] S. Butler and P. Harrowell, The Origin of Glassy Dynamics in the 2D Facilitated Kinetic IsingModel. J. Chem. Phys., 1991. 95(6): p. 4454-4465.

[72] M. Y. Efremov, S. S. Soofi, et al., Vacuum ellipsometry as a method for probing glass transition in thin polymer films. Review of Scientific Instruments, 2008. 79(4): p. 043903.

[73] O. M. Corbino, Thermische Oszillationen wechselstromdurchflossener Lampen mit dünnem Faden und daraus sich ergebende Anwesenheit geradzahliger Oberschwingungen. Physik. Zeitschr., 1910. 11: p. 413-417.

[74] Y. Kraftmakher, Modulation Calorimetry and Related Techniques. Phys. Rep., 2002. 356(1-2): p. 1-117.

[75] Y. A. Kraftmakher, Modulation method of heat capacity measurement. Zhurnal prikladnoj mechaniki i techniyeceskoj fiziki, 1962. 5: p. 176-180.

[76] P. Sullivan and G. Seidel, An AC Temperature Technique for measuring Heat Capacities. Ann. Acad. Sci. Fennicae, 1966. A(210): p. 58-62.

[77] P. F. Sullivan and G. Seidel, Steady-State, AC-Temperature Calorimetry. Phys. Rev., 1968. 173(3): p. 679-685.

[78] I. V. Velichkov, On the Problem of Thermal Link Resistances in A.C. Calorimetry. Cryogenics, 1992. 32(3): p. 285-290.

[79] R. L. Greene, C. N. King, et al., Specific Heat of Granular Aluminum Films. Phys. Rev. B, 1972. 6(9): p. 3297-3305.

[80] O. Riou, J. F. Durastanti, and Y. Sfaxi, Determination of Proper Frequency Range for Accurate Heat Capacity Measurement by AC Microcalorimeter. Superlat. Microstruc., 2004. 35(3-6): p. 353-361.

[81] R. Bachmann, F. J. DiSalvo, et al., Heat capacity measurements on small samples at low temperatures. Rev. Sci. Instrum., 1972. 43(2): p. 205-214.

[82] F. Fominaya, T. Fournier, et al., Nanocalorimeter for High-Resolution Measurements of LowTemperature Heat- Capacities of Thin-Films and Single-Crystals. Rev. Sci. Instrum., 1997. 68(11): p. 4191-4195.

[83] O. Riou, P. Gandit, et al., Very Sensitive Microcalorimetry Technique for Measuring SpecificHeat of mu g Single-Crystals. Rev. Sci. Instrum., 1997. 68(3): p. 1501-1509.

[84] S. Tagliati, A. Rydh, and R. Xie, Membrane-Based Calorimetry for Studies of Sub-Microgram Samples. J. Phys. Conf. Ser., 2009. 150(5): p. 052256.

[85] A. A. Minakov, Y. V. Bugoslavsky, and C. Schick, Improvement of AC Calorimetry for Simultaneous Measurements of Heat Capacity and Thermal Conductivity of Polymers. Thermochim. Acta, 1998. 317(2): p. 117-131.

[86] A. A. Minakov, S. B. Roy, et al., Thin-film Alternating Current Nanocalorimeter for Low Temperatures and High Magnetic Fields. Rev. Sci. Instrum., 2005. 76(4): p. 043906.

[87] M. Merzlyakov, Integrated Circuit Thermopile as a New Type of Temperature Modulated Calorimeter. Thermochim. Acta, 2003. 403(1): p. 65-81.

[88] G. S. Dixon, S. G. Black, et al., A Differential AC Calorimeter for Biophysical Studies. Anal. Biochem., 1982. 121(1): p. 55-61.

[89] H. Huth, A. A. Minakov, et al., Differential AC-Chip Calorimeter for Glass Transition Measurements in Ultra Thin Polymeric Films. Eur. Phys. J.-Spec. Top., 2007. 141: p. 153-160.

[90] D. Zhou, H. Huth, et al., Calorimetric Glass Transition of Poly(2,6-dimethyl-1,5-phenylene oxide) Thin Films. Macromolecules 2008. 41(20): p. 7662-7666. 
[91] W. Jiang, M. Du, et al., Calorimetric Study of Blend Miscibility of Polymers Confined in UltraThin Films. Eur. Phys. J.-Spec. Top., 2010. 189: p. 187-195.

[92] T. W. Kenny and P. L. Richards, AC Calorimeter for Measurements of Adsorbed Gases on Metal Films at 4He Temperatures. Rev. Sci. Instrum., 1990. 61(2): p. 822-829.

[93] D. W. Denlinger, E. N. Abarra, et al., Thin-Film Microcalorimeter for Heat Capacity Measurements from $1.5 \mathrm{~K}$ to $800 \mathrm{~K}$. Rev. Sci. Instrum., 1994. 65(4): p. 946-958.

[94] S. L. Lai, G. Ramanath, et al., High-Speed (10(4)-Degrees-C/S) Scanning Microcalorimetry with Monolayer Sensitivity (J/M(2)). Appl. Phys. Lett., 1995. 67(9): p. 1229-1231.

[95] D. R. Queen and F. Hellman, Thin Film Nanocalorimeter for Heat Capacity Measurements of $30 \mathrm{~nm}$ Films. Rev. Sci. Instrum., 2009. 80(6): p. 063901.

[96] S. A. Adamovsky, A. A. Minakov, and C. Schick, Scanning Microcalorimetry at High Cooling Rate. Thermochim. Acta, 2003. 403(1): p. 55-63.

[97] A. F. Lopeandía, E. Leon-Gutierrez, et al., Nanocalorimetric high-temperature characterization of ultrathin films of a-Ge. Materials Science in Semiconductor Processing, 2006. 9: p. 806-811.

[98] E. Zhuravlev and C. Schick, Fast scanning power compensated differential scanning nanocalorimeter: 1. The device. Thermochimica Acta, 2010. 505(1-2): p. 1-13.

[99] A. W. van Herwaarden, Overview of Calorimeter Chips for Various Applications. Thermochim. Acta, 2005. 432(2): p. 192-201.

[100] A. Minakov, J. Morikawa, et al., Temperature Distribution in a Thin-Film Chip utilized for Advanced Nanocalorimetry. Meas. Sci. Technol., 2006. 17(1): p. 199-207.

[101] COMSOL, COMSOL Multiphysics AB, www.comsol.com. COMSOL Multiphysics AB (website: www.comsol.com).

[102] XENSOR, Gas nano calorimeter by Xensor Integration bv, Delft, The Netherlands. Gas nano calorimeter by Xensor Integration bv, Delft, The Netherlands (website: www.xensor.nl).

[103] P. M. Sarro, A. W. Van Herwaarden, and W. Van der VlistT, A Silicon-Silicon Nitride Membrane Fabrication Process for smart Thermal Sensors. Sens. Actuators, A, 1994. 42(1-3): p. 666-671.

[104] B. Revaz, B. L. Zink, and F. Hellman, Si-N membrane-based microcalorimetry: Heat capacity and thermal conductivity of thin films. Thermochim. Acta, 2005. 432(2): p. 158-168.

[105] C. H. Mastrangelo, T. Yu-Chong, and R. S. Muller, Thermophysical Properties of Low-residual Stress, Silicon-rich, LPCVD Silicon Nitride Films. Sens. Actuators: Phys. A, 1990. 23(1-3): p. 856-860.

[106] B. L. Zink and F. Hellman, Specific Heat and Thermal Conductivity of Low-Stress Amorphous SiN Membranes. Solid State Commun., 2004. 129(3): p. 199-204.

[107] G. C. M. Meijer and A. W. v. Herwaarden, Thermal Sensors. 1994, Bristol, U.K.: Institute of Physics Publishing. 304.

[108] R. W. Powell, C. Y. Ho, and P. E. Liley, Thermal Conductivity of Selected Materials. NIST, 1966.

[109] P. Richet, Y. Bottinga, et al., Thermodynamic properties of quartz, cristobalite and amorphous SiO2: drop calorimetry measurements between 1000 and $1800 \mathrm{~K}$ and a review from 0 to 2000 K. Geochimica et Cosmochimica Acta, 1982. 46(12): p. 2639-2658.

[110] M. L. V. Ramires, C. A. Nietro_de_C, et al., Reference Data for the Thermal Conductivity of Saturated Liquid Toluene Over a Wide Range of Temperature. JPCRD, 2000. 29(2): p. 133-139.

[111] O. Yamamuro, I. Tsukushi, et al., Calorimetric Study of Glassy and Liquid Toluene and Ethylbenzene - Thermodynamic Approach to Spatial Heterogeneity in Glass-Forming Molecular Liquids. J. Phys. Chem. B, 1998. 102(9): p. 1605-1609.

[112] M. O. McLinden, Liquid Density Standard Over Wide Ranges of Temperature and Pressure Based on Toluene: A Reprint from the Journal of Research of the NIST. 2009: DIANE Publishing Company.

[113] S. Weyer, A. Hensel, and C. Schick, Phase Angle Correction for TMDSC in the Glass-Transition Region. Thermochim. Acta, 1997. 305: p. 267-275. 
[114] Z. Chen and R. Richert, Dynamics of glass-forming liquids. XV. Dynamical features of molecular liquids that form ultra-stable glasses by vapor deposition. Journal of Chemical Physics, 2011. 135(12): p. 124515.

[115] M. Hatase, M. Hanaya, et al., Discovery of Homogeneous-Nucleation-Based Crystallization in simple Glass-Forming Liquid of Toluene below its Glass-Transition Temperature. J. Non-Cryst. Solids, 2002. 307: p. 257-263.

[116] A. Mandanici, W. Huang, et al., Dynamics of Glass-Forming Liquids. XII. Dielectric Study of Primary and Secondary Relaxations in Ethylcyclohexane. J. Chem. Phys., 2008. 128(12): p. 124505.

[117] A. Torrisi, C. K. Leech, et al., Solid Phases of Cyclopentane: Combined Experimental and Simulation Study. J. Phys. Chem. B, 2008. 112(12): p. 3746-3758.

[118] R. Jakobi, E. Gmelin, and K. Ripka, High-Precision Adiabatic Calorimetry and the Specific Heat of Cyclopentane at low Temperature. J. Therm. Anal., 1993. 40(3): p. 871-876.

[119] K. K. Kelley, The Heat Capacity of Toluene from 14 Degrees K. to 298 Degrees K. The Entropy and the Free Energy of Formation. J. Am. Chem. Soc., 1929. 51: p. 2738-2741.

[120] D. W. Scott, W. T. Berg, et al., Toluene - Thermodynamic Properties, Molecular Vibrations, and Internal Rotation. J. Phys. Chem., 1962. 66(5): p. 911-914.

[121] G. Hinze and H. Sillescu, H-2 Nuclear Magnetic Resonance Study of Supercooled Toluene: Slow and Fast Processes above and below the Glass Transition. J. Chem. Phys., 1996. 104(1): p. 314-319.

[122] A. Doss, G. Hinze, et al., Dielectric Relaxation in the Fragile Viscous Liquid State of Toluene. J. Chem. Phys. , 1997. 107(6): p. 1740-1743.

[123] A. Kudlik, C. Tschirwitz, et al., Slow Secondary Relaxation Process in Supercooled Liquids. Europhys. Lett., 1997. 40(6): p. 649-654.

[124] G. Floudas, G. Fytas, and W. Brown, Solvent Mobility in Poly(Methyl Methacrylate) Toluene Solutions by Depolarized and Polarized-Light Scattering. J. Chem. Phys., 1992. 96(3): p. 21642174.

[125] G. Floudas, W. Steffen, and E. W. Fischer, Solvent and Polymer Dynamics in Concentrated Polystyrene Toluene Solutions. J. Chem. Phys., 1993. 99(1): p. 695-703.

[126] E. Rossler and H. Sillescu, H2 NMR-Study of Supercooled Toluene. Chem. Phys. Lett., 1984. 112(1): p. 94-98.

[127] D. Morineau, Y. Xia, and C. Alba-Simionesco, Finite-Size and Surface Effects on the Glass Transition of Liquid Toluene confined in Cylindrical Mesopores. J. Chem. Phys. , 2002. 117(19): p. 8966-8972.

[128] M. Cutroni and A. Mandanici, Mechanical Response of Supercooled Ethylbenzene at Ultrasonic Frequencies. Philos. Mag., 2004. 84(13-16): p. 1499-1506.

[129] Z. Chen and R. Richert, Dynamics of Glass-forming Liquids. XV. Dynamical Features of Molecular Liquids that form Ultra-stable Glasses by Vapor Deposition. J. Chem. Phys., 2011. 135(12): p. 124515.

[130] B. Jakobsen, T. Hecksher, et al., Communication: Identical Temperature Dependence of the Time Scales of several Linear-Response Functions of two Glass-Forming Liquids. J. Chem. Phys., 2012. 136(8): p. 081102.

[131] A. Lion, M. Engelhard, and M. Johlitz, Thermomechanical and Calorimetric Behaviours of Supported Glass-Forming Films: A Study based on Thermodynamics with Internal Variables. Thin Solid Films, 2012. 522: p. 441-451.

[132] M. Tress, M. Erber, et al., Glassy Dynamics and Glass Transition in Nanometric Thin Layers of Polystyrene. Macromolecules, 2010. 43(23): p. 9937-9944.

[133] M. Erber, M. Tress, et al., Glassy Dynamics and Glass Transition in Thin Polymer Layers of PMMA Deposited on Different Substrates. Macromolecules, 2010. 43(18): p. 7729-7733.

[134] J. A. Forrest and K. Dalnoki-Veress, The Glass Transition in Thin Polymer Films. Adv. Colloid Interfac., 2001. 94(1-3): p. 167-196. 
[135] P. G. Wolynes, Spatiotemporal Structures in Aging and Rejuvenating Glasses. Proc. Natl. Acad. Sci. U.S.A., 2009. 106(5): p. 1353-1358.

[136] A. Sepulveda, S. F. Swallen, et al., Stable glasses of indomethacin and $a, a, b$-trisnaphthylbenzene transform into ordinary supercooled liquids. J. Chem. Phys., 2012: p. submitted.

[137] K. Dawson, L. A. Kopff, et al., Molecular Packing in Highly Stable Glasses of Vapor-Deposited tris-Naphthylbenzene Isomers. J. Chem. Phys., 2012. 136(9): p. 094505.

[138] K. L. Kearns, M. D. Ediger, et al., One Micrometer Length Scale Controls Kinetic Stability of Low-Energy Glasses. J. Phys. Chem. Lett., 2010. 1(1): p. 388-392.

[139] S. S. Chang and A. B. Bestul, Heat Capacity and Thermodynamic Properties of o-Terphenyl Crystal, Glass, and Liquid. J. Chem. Phys. , 1972. 56(1): p. 503-516.

[140] S. F. Swallen, K. L. Kearns, et al., Molecular View of the Isothermal Transformation of a Stable Glass to a Liquid. J. Chem. Phys., 2008. 128(21): p. 214514.

[141] J. D. Stevenson and P. G. Wolynes, On the Surface of Glasses. J. Chem. Phys., 2008. 129(23): p. 234514.

[142] M. D. Ediger, Spatially Deterogeneous Dynamics in Supercooled Liquids. Annu. Rev. Phys. Chem., 2000. 51: p. 99-128.

[143] R. Richert, Heterogeneous Dynamics in Liquids: Fluctuations in Space and Time. J. Phys.Condens. Mat., 2002. 14(23): p. R703-R738.

[144] S. Singh, M. D. Ediger, and J. J. de Pablo, Ultrastable Glasses from In Silico Vapour Deposition. Nat Mater, 2013. 12(2): p. 139-144.

[145] K. Ishii, H. Nakayama, et al., Behavior of Glass and Supercooled Liquid Alkylbenzenes Vapordeposited on cold Substrates: Toward the Understanding of the Curious Light Scattering observed in some Supercooled Liquid States. B. Chem. Soc. Jpn., 2009. 82(10): p. 1240-1247.

[146] K. Ishii, H. Nakayama, et al., Excess Volume of Vapor-Deposited Molecular Glass and its Change due to Structural Relaxation: Studies of Light Interference in Film Samples. J. Phys. Chem. B, 2003. 107(3): p. 876-881.

[147] I. Cohen, A. Ha, et al., A Low-Temperature Amorphous Phase in a Fragile Glass-Forming Substance. J. Phys. Chem., 1996. 100(20): p. 8518-8526.

[148] H. Tanaka, R. Kurita, and H. Mataki, Liquid-Liquid Transition in the Molecular Liquid Triphenyl Phosphite. Phys. Rev. Lett., 2004. 92(2): p. 025701.

[149] S. Aasland and P. F. McMillan, Density-driven Liquid-Liquid Phase-Separation in the System AL2O3-Y2O3. Nature, 1994. 369(6482): p. 633-636.

[150] G. N. Greaves, M. C. Wilding, et al., Detection of First-Order Liquid/Liquid Phase Transitions in Yttrium Oxide-Aluminum Oxide Melts. Science, 2008. 322(5901): p. 566-570.

[151] O. Mishima, L. D. Calvert, and E. Whalley, An Apparently 1st-Order Transition between 2 Amorphous Phases of Ice induced by Pressure. Nature, 1985. 314(6006): p. 76-78.

[152] B. Champagnon, C. Martinet, et al., Polyamorphism: Path to New High Density Glasses at Ambient Conditions. J. Non-Cryst. Solids, 2007. 353(44-46): p. 4208-4211.

[153] S. Capponi, S. Napolitano, and M. Wubbenhorst, Supercooled Liquids with Enhanced Orientational Order. Nat. Commun., 2012. 3: p. 1233. 


\section{List of Figures}

Figure 1: Schematic enthalpy over temperature plot for a glass forming material..... 4

Figure 2: Frequency dependence of the real and the imaginary parts of the dielectric permittivity in glycerol. 6

Figure 3: Total specific heat capacity $\left(c_{p, t o t a l}\right)$, real $\left(c_{p}\right)$ and imaginary part $\left(c_{p}{ }^{\prime \prime}\right)$ of the complex specific heat capacity of polystyrene ...............................................

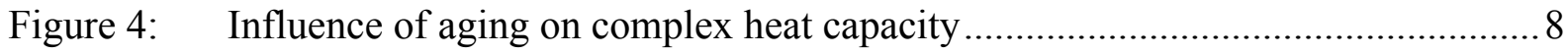

Figure 5: Angell plot for three different fragilities .....................................................

Figure 6: The configurational enthalpy of vapor-deposited and liquid quenched samples of butyronitrile 12

Figure 7: Enthalpy curves, $T_{f}$ and $T_{\text {on }}$ for samples of IMC deposited at various $T_{\text {substrate } \ldots} 15$ Figure 8: $\quad$ Enthalpy curves, $T_{f}, T_{\text {on }}$ for samples of IMC deposited at various deposition rates

Figure 9: Inhomogeneous evolution of $\mathrm{d}-\mathrm{TNB} / \mathrm{h}-\mathrm{TNB}$ concentration profiles for a stable glass and transformation of stable glasses of IMC for different film thicknesses.

Figure 10: Photograph of the peripheral chamber setup ................................................... 19

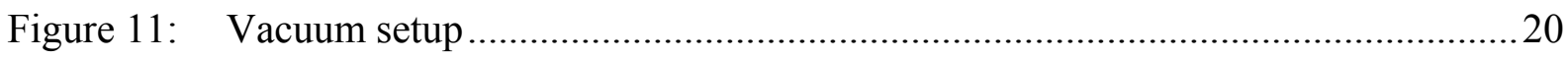

Figure 12: CAD drawing of the copper parts of the sample holder..................................2

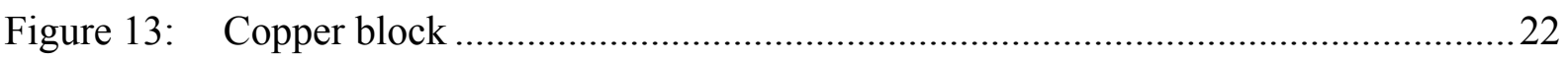

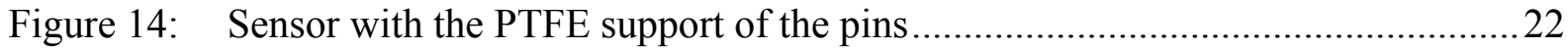

Figure 15: Schematic sketch of the setup investigated by Sullivan and Seidel ...................25

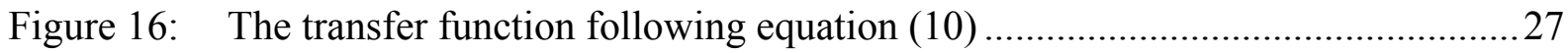

Figure 17: Schematic sketch of the setup as investigated by Velichkov ............................22

Figure 18: Three different solutions for the transfer function as calculated from the model proposed by Velichkov.

Figure 19: The transfer function of a membrane based calorimeter celltaking into account the thermal diffusivity of the substrate and the effective thermal link to the sample.

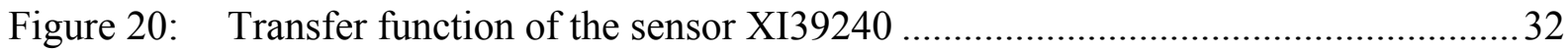

Figure 21: Single sensor temperature amplitude as a function of thermal frequency and

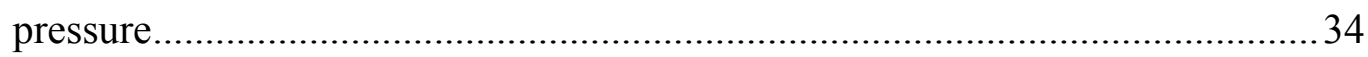

Figure 22: Photographs of the calorimetric chip sensor XI39390 …................................. 35

Figure 23: Scheme of the electronic setup of the differential AC calorimeter..................... 36 
Figure 24: Differential temperature amplitude of the differential setup in vacuum and at $T_{\text {substrate }}=105 \mathrm{~K}$ as a function of frequency

Figure 25: Complex temperature amplitude of the reference and sample sensors visualized in the complex plane

Figure 26: Evolution of the differential temperature amplitude during deposition of a thin toluene film 39

Figure 27: Splitting of the membrane 40

Figure 28: Schematic picture of the semi-analytical model 40

Figure 30: Scheme of the central part of the membrane 43

Figure 31: Steady state comparison between the experimental data and the model data .....45

Figure 32: Comparison of simulated and experimental data points for an empty sensor and a sensor loaded with a $390 \mathrm{~nm}$ toluene sample

Figure 33: Differential temperature amplitude and phase angle between temperature and power for the differential signal for a $390 \mathrm{~nm}$ toluene film

Figure 34: Experimental and modeled data for three films of slowly cooled toluene glasses of different thickness

Figure 35: measured differential temperature amplitude and modeled data with different thicknesses.

Figure 36: DC voltage and differential temperature amplitude over heater resistance during heating of a thin toluene sample starting above the glass transition 50

Figure 37: Measured differential temperature amplitude and phase for heating and cooling of toluene over Pt100 temperature

Figure 38: Differential temperature amplitude and phase over heater resistance for a thin toluene sample

Figure 39: 3D and 2D drawing of cyclopentane

Figure 40: Phase angle of the complex reversing heat capacity of cyclopentane over heater resistance 57

Figure 41: Structure of the toluene molecule. 58

Figure 42: Plot of VFTs and data of the structural relaxation time of toluene found in the literature 59

Figure 43: Structure of the ethylbenzene molecule. 60

Figure 44: Plot of the VTF functions for ethylbenzene found in the literature 61

Figure 45: Determined calibration points for the three cyclopentane transitions together with a second-order polynominal fit function. 
Figure 46: Heater resistance and DC voltage over time for heating a $400 \mathrm{~nm}$ film of toluene from the glass into the melt 64

Figure 47: The determination of the sensitivity of the thermopiles of the used sensors ..... 66

Figure 49: Temperature program for the dynamic glass transition scans of toluene. .68

Figure 50: The glass transition of a $2 \mathrm{~nm}$ thin film of toluene 69

Figure 51: (a) $\Delta C_{p} / C_{p}$ (glass) over film thickness for different depositions of thin films of ordinary glasses of toluene. (b) Thickness dependence of the step height at the glass transition. 70

Figure 52: Thickness dependence of the dynamic glass transition temperature at $20 \mathrm{~Hz} \ldots . .71$

Figure 54: Heat capacity of the first heating curves of as-deposited stable glasses of toluene deposited at substrate temperatures ranging from $71 \mathrm{~K}$ to $109 \mathrm{~K}$ and ethylbenzene deposited from $65 \mathrm{~K}$ to $100 \mathrm{~K}$ measured with the He-cooled device. 75

Figure 55: Onset temperature $\left(T_{o n}\right)$ and fractional $C_{p}$ decrease of as-deposited stable glasses of (a) toluene and (b) ethylbenzene for different substrate temperatures..... .76

Figure 56: Heat capacity of as-deposited stable glasses of (a) toluene and (b) ethylbenzene deposited at deposition rates spanning more than three orders of magnitude.....78

Figure 57: The onset temperature $\left(T_{o n}\right)$ and fractional $C_{p}$ decrease of as-deposited stable glasses of (a) toluene and (b) ethylbenzene for different deposition rates 78

Figure 58: Isothermal transformation kinetics of stable glasses of (a) toluene and (b) ethylbenzene to the SCL for different transformation temperatures 80

Figure 59: The logarithm of the isothermal transformation time $t_{\text {transform }}$ to the structural relaxation time $\tau_{\alpha}$, as a function of the negative of $\log \left(\tau_{\alpha}\right)$ for three different substances.....

Figure 60: $\log \left(t_{\text {transform }} / \tau_{\alpha}\right)$ over (a) $-\log \left(\tau_{\alpha}\right)$ and (b) film thickness for stable glasses of toluene

Figure 61: Difference between $T_{g, d y n}$ of toluene and ethylbenzene determined from the asdeposited stable glass and from the ordinary glass as a function of deposition

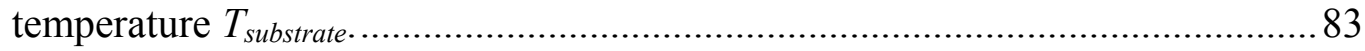

Figure 62: (a) low and (b) high frequency measurement of a $75 \mathrm{~K}$ deposition of ethylbenzene. 84

Figure 63: Kinetic stability as a function of fractional $C_{p}$ decrease 86

Figure 65: Electrical setup to measure the DC thermopile voltage..... 114 
Figure 66: Amplification circuit of the 4 to $20 \mathrm{~mA}$ output $(11-12)$ of the OMRON E5CK temperature MOSPEC TIP 121 silicon transistor. .......................................... 115

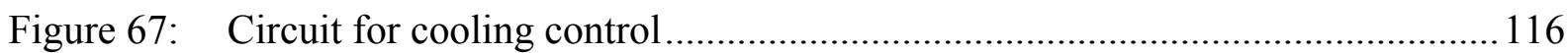

Figure 68: Electrical scheme for switching the electro-pneumatic angle valve for deposition

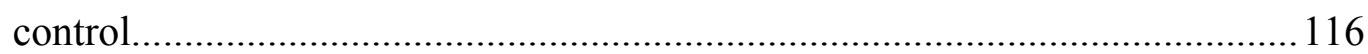

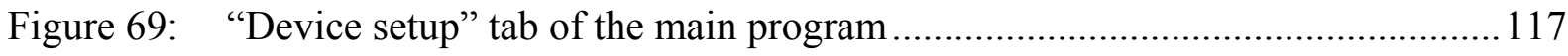

Figure 70: Sub-program for displaying the amplified sinusoidal voltages........................ 118

Figure 71: "Measurement setup" tab of the main program .............................................. 119

Figure 72: Sub-program for temperature program setup ............................................. 120

Figure 73: Pop-up in the sub-program if a temperature scan was chosen ......................... 120

Figure 74: Pop-up window to set the parameters for the lock-in amplifier....................... 121

Figure 75: Example of a cooling-heating temperature profile ........................................ 121

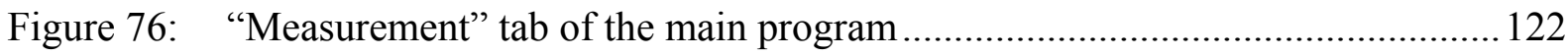

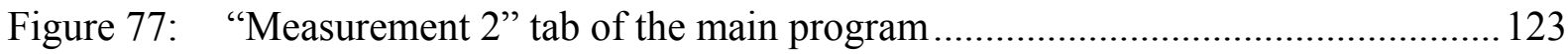




\section{Table of Abbreviations}

\begin{tabular}{|c|c|}
\hline$A C$ & alternating current \\
\hline$A D C$ & analogue-to-digital converter \\
\hline$B$ & VFT-parameter \\
\hline$C_{0, e f f}$ & effective apparent addenda heat capacity \\
\hline$C_{a p}$ & apparent heat capacity \\
\hline$c_{g a s}$ & heat capacity of the gas \\
\hline$C_{h}$ & heat capacity of the heater \\
\hline$C_{o}$ & apparent addenda heat capacity \\
\hline$C_{p}$ & heat capacity at constant pressure \\
\hline$c_{p}$ & specific heat capacity at constant pressure \\
\hline$c_{p}^{\prime}$ & real part of the complex heat capacity \\
\hline$c_{p}^{\prime \prime}$ & imaginary part of the complex heat capacity \\
\hline$C_{s}$ & sample heat capacity \\
\hline$C_{t}$ & heat capacity of the thermometer \\
\hline$C V D$ & chemical vapor deposition \\
\hline$D$ & VFT-parameter \\
\hline$D C$ & direct current \\
\hline$D M M$ & digital multimeter \\
\hline$D R S$ & depolarized Rayleigh scattering \\
\hline$d_{s}$ & sample thickness \\
\hline$D S$ & dielectric susceptibility \\
\hline$D S C$ & differential scanning calorimetry \\
\hline$E$ & energy \\
\hline$f$ & frequency \\
\hline$F(x)$ & functional \\
\hline FEM & finite elements simulation \\
\hline$f_{t h}$ & thermal frequency \\
\hline$G$ & heat exchange coefficient \\
\hline$H$ & enthalpy \\
\hline
\end{tabular}




$\begin{array}{ll}H D G & \text { high density glass } \\ H D L & \text { high density liquid } \\ I M C & \text { indomethacin } \\ k_{B} & \text { Boltzmann constant } \\ K_{b} & \text { thermal link from bath-to-sample } \\ K_{b, e f f} & \text { effective thermal link from substrate to bath } \\ k_{g a s} & \text { wave number } \\ K_{h} & \text { thermal link from heater-to-sample } \\ K_{s} & \text { thermal link from sample to substrate } \\ K_{t} & \text { thermal link from thermometer-to-sample } \\ L D G & \text { low density glass } \\ L D L & \text { low density liquid } \\ L N 2 & \text { liquid nitrogen } \\ m & \text { fragility } \\ N M R & \text { nuclear magnetic resonance } \\ \hat{p}_{0} & \text { ordinary glass } \\ p_{0} & \text { power corresponding to heat loss of sample } \\ & \text { heat transfer coefficient } \\ & \text { power amplitude } \\ & \end{array}$

PMMA polymethylmethacrylate

PS polystyrene

PTFE Teflon

PVD physical vapor deposition

Q heat flux

QCM quartz crystal microbalance

$R_{h} \quad$ resistance of the heater

$R_{i} \quad$ resistance of pre-resistor

RTD resistive temperature device

$R_{T g} \quad$ heater resistance at the glass transition temperature 
S

$S C L$

$S G$

SIMS

$T$

$t$

$T_{0}$

$T_{a}$

$T_{\text {avg }}$

$T_{b}$

$T_{f}$

$T_{g}$

$T_{g, d y n}$

$T_{\text {gas }}(t, r)$

$T_{h}$

$T_{k}$

$T_{m}$

TMDSC

$T N B$

$T_{\text {on }}$

$T_{s}$

$T_{\text {substrate }}$

$T_{t}$

$t_{\text {transform }}$

$T_{\text {transform }}$

$U_{h}$

$U H V$

$U_{i}$

V

VFT entropy

supercooled liquid

stable glass

secondary ion mass spectroscopy

temperature

time

Vogel-temperature

annealing temperature

mean sample temperature

bath temperature

fictive temperature

glass transition temperature

dynamic glass transition temperature

thermal wave function through gas

temperature of the heater

Kauzmann temperature

melting temperature

temperature modulated differential scanning calorimetry

tris-naphthylbenzene

onset temperature

sample temperature

substrate temperature

temperature of the thermometer

transformation time

transformation temperature

voltage drop over heater

ultra high vacuum

voltage drop over pre-resistance

volume

Vogel-Fulcher-Tamman 


\begin{tabular}{|c|c|}
\hline$V Q$ & vapor quenched \\
\hline$W A X S$ & wide angle $\mathrm{x}$-ray scattering \\
\hline$\alpha$ & expansivity \\
\hline$\alpha_{s}$ & effective thermal thickness \\
\hline$\beta$ & heating rate \\
\hline$\beta_{K W W}$ & Kohlrausch-Williams-Watt-exponent \\
\hline$\Delta C_{p}$ & heat capacity step at the glass transition \\
\hline$\Delta \theta_{0}$ & complex differential temperature of the loaded system \\
\hline$\Delta \theta_{00}$ & complex differential temperature of the empty system \\
\hline$\theta$ & modulated temperature \\
\hline$\theta_{0}$ & amplitude of the temperature oscillation \\
\hline$\kappa$ & compressibility \\
\hline$\lambda_{\text {gas }}$ & thermal conductivity of the gas \\
\hline$\rho$ & density \\
\hline$\rho_{\text {gas }}$ & density of the gas \\
\hline$\tau$ & relaxation time of the sample \\
\hline$\tau_{b}$ & substrate-to-heater relaxation time \\
\hline$\tau_{s}$ & sample-to-bath relaxation time \\
\hline$\tau_{t}$ & substrate-to-thermometer relaxation time \\
\hline$\tau_{\alpha}$ & structural relaxation time \\
\hline$\Phi$ & phase angle \\
\hline$\Phi_{S G}$ & fraction of OG-to-SG-transformation \\
\hline$\omega$ & angular frequency \\
\hline
\end{tabular}




\section{Appendix}

\section{Appendix A: Detailed Experimental setup}

\section{Appendix A.1: Copper block}

The copper block (see Figure 12) has a height of $45 \mathrm{~mm}$ and a diameter of $71 \mathrm{~mm}$ with 4 passing holes where sensors can be placed and a radial notch with a depth of $11 \mathrm{~mm}$ for some copper rings to simplify the installation of the sensors. For the same purpose, 10-pin sockets are placed, soldered to the electrical wires and fixed inside the copper block using a copper ring (again with 4 passing holes) placed in the notch that clamps the sockets. Another copper ring (again with 4 passing holes) is mounted on top of the inner ring to complete the notch. This ring is used to clamp the sensors to another ring to protect the sensors from destruction during installation. A final copper ring that has the same outer diameter as the copper block, an inner diameter of $40.4 \mathrm{~mm}$ and a height of $16 \mathrm{~mm}$ is used to shield the reference sensor from deposition. This ring also brings the sensors into the right position using notches where the pointing nose on the edge of the housing perfectly fits and to mount an electronically driven shutter that can be used to close the hole to the sample sensor and protect it from uncontrolled deposition. This last and biggest copper ring is constructed for only two sensors, a reference sensor and a sample sensor, since three sample sensors (as planned previously) are challenging regarding the installation of shutters combined with the space available. The shutter itself consists of a common electromotor from an old CD-ROM drive and a PTFE branch to cover the hole to the sample sensor (Figure 13). 


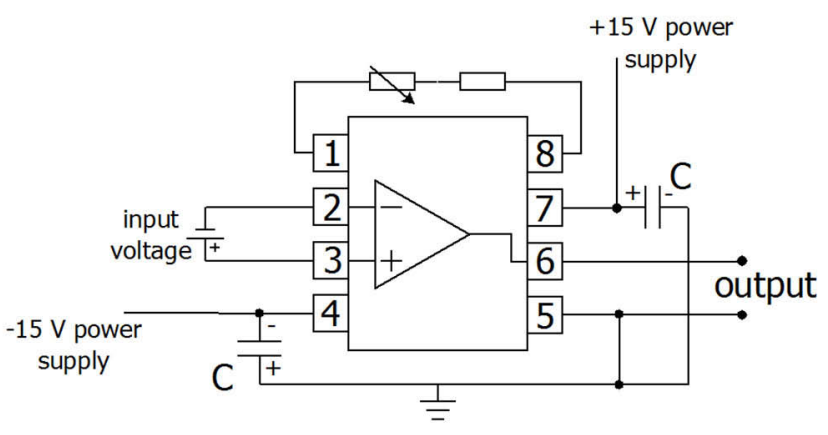

Figure 64: Sketch of the amplification circuit to amplify the voltage drops at the heater resistance $R_{h}$ and at the known resistance $R_{i}$. The used AD620 instrumentation amplifier is set to an amplification of factor 2 . The capacitances $C=0.1 \mu \mathrm{F}$ are for stabilization purposes.

\section{Appendix A.3: Measurement of DC thermopile voltage}

The thermopile voltage is the superposition of the oscillating temperature amplitude and the DC overheating of the membrane coming from the non-oscillating difference between the hot and cold junctions of the thermopiles. The DC thermopile voltage is taken from the signal monitor (SIG MON) output of the lock-in amplifier. Since this voltage still includes the oscillating part, a low pass is hereafter installed and is dimensioned to blind out the thermal frequency that is used (Figure 65). The RC filter consists of $\mathrm{R}=3900 \Omega$ and $C=470 \mu \mathrm{F}$ which leads to a cutoff frequency of $f_{t}=0.09 \mathrm{~Hz}$. Even the lowest frequency used in this work $(0.1 \mathrm{~Hz}$ oscillator frequency, or $0.2 \mathrm{~Hz}$ thermal frequency) is reasonably suppressed. The DC thermopile voltage is then measured by an Agilent 34410A digital multimeter (DMM).

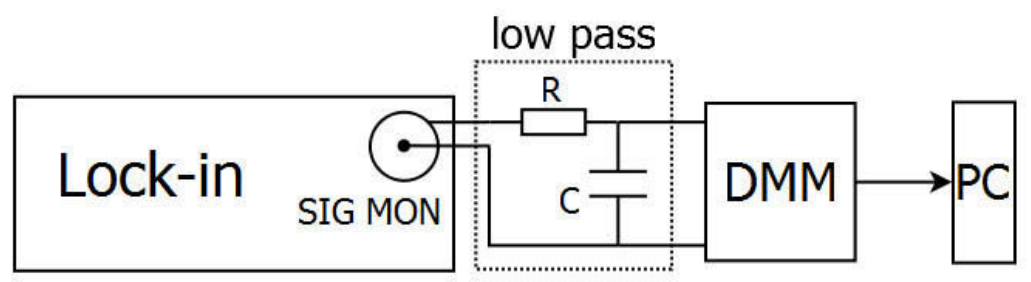

Figure 65: Electrical setup to measure the DC thermopile voltage. The low pass components are $R=3900 \Omega$ and $C=470 \mu \mathrm{F}$. 


\section{Appendix A.4: Temperature control setup}

The power inputs of the controller are connectors 4 and 5 as shown in Figure 66. A Pt100 resistive thermometer is screwed in the copper block to measure the block temperature. This thermometer is connected to inputs 6,7 and 8. Depending on some of the controller parameters, the 4 to $20 \mathrm{~mA}$ output (11 and 12) of the controller provides a certain current to the amplification circuit as shown in Figure 66. This current is amplified using a MOSPEC TIP 121 silicon transistor and a $48 \mathrm{~V}$ power supply to heat the cartridge heaters with maximum $40 \mathrm{~W}$.

The temperature controller runs cooling and heating separately. If heating is on cooling is off and vice versa. The set PID parameters are valid for both heating and cooling. To cool the copper block a mechanical relay, pins 9 and 10 of the controller, is switching a CRYDOM TD 2410 solid state relay (SSR) by disrupting the circuit for the SSR 5 VDC power supply. The SSR itself switches the 230 VAC input voltage of a 12 VDC power supply which again switches the electro-magnetic valve at the liquid nitrogen dewar (see Figure 67). The auxiliary output, pins 2 and 3 , is not needed and thus vacant.

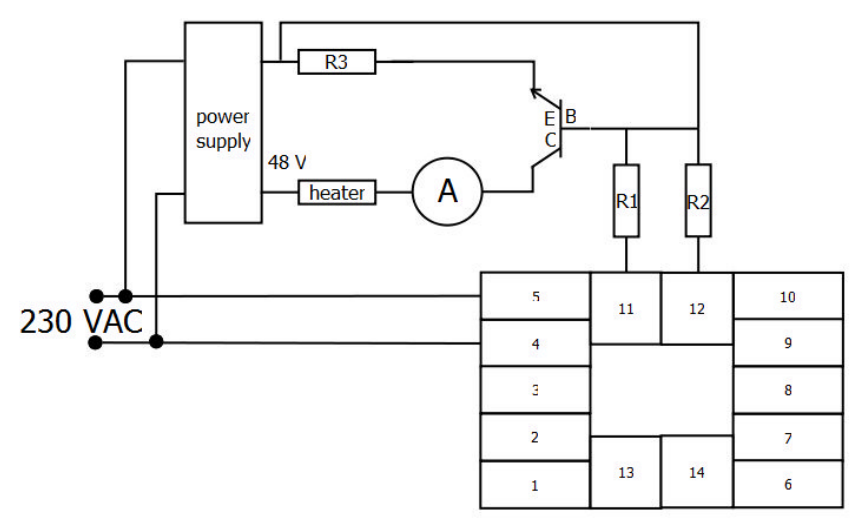

Figure 66: Amplification circuit of the 4 to $20 \mathrm{~mA}$ output $(11-12)$ of the OMRON E5CK temperature controller used for providing power to the cartridge heaters. The resistances are as follows: $R 1=330 \Omega, R 2$ (base resistance) $=180 \Omega, R 3$ (emitter resistance) $=2.2 \Omega$ and the cartridge heater resistance is about $40 \Omega$. Thus the current is limited to about $1 \mathrm{~A}$. An ampere meter for visualization of the current is connected in series. This circuit is using an MOSPEC TIP 121 silicon transistor. 


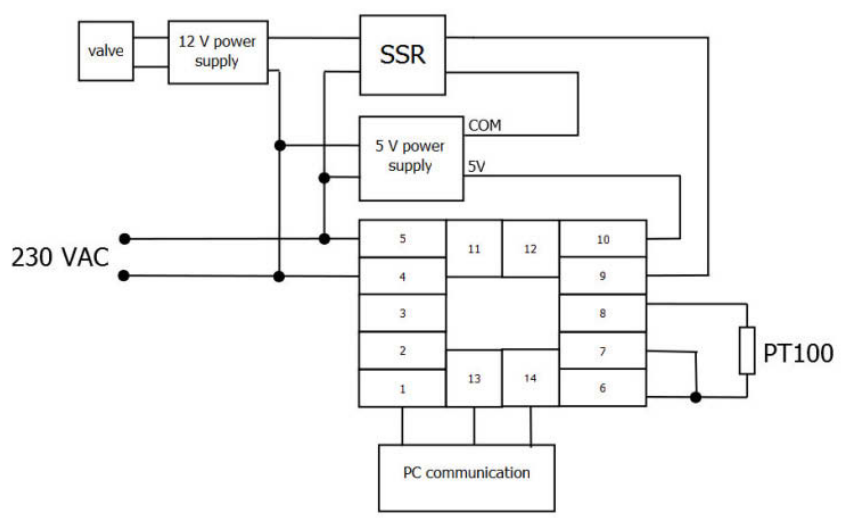

Figure 67: Circuit for cooling control. If the controller relay (9 and 10) is closed the SSR is switching on a power supply which again provides $12 \mathrm{~V}$ for opening the valve at the dewar.

\section{Appendix A.5: Deposition control setup}

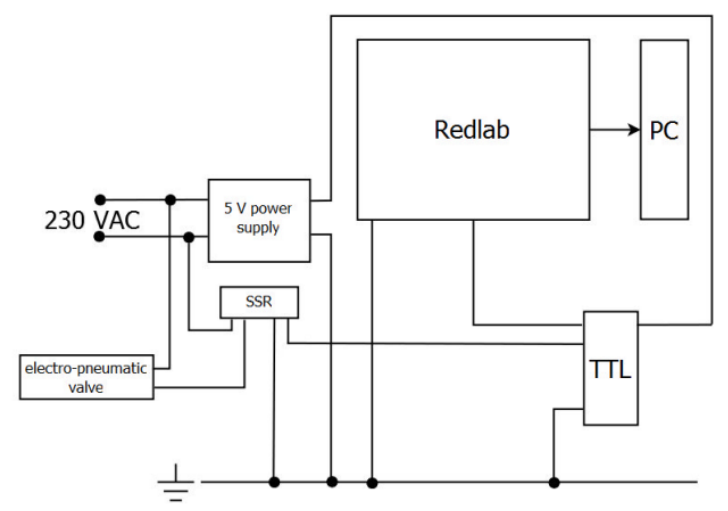

Figure 68: Electrical scheme for switching the electro-pneumatic angle valve for deposition control. The output voltage at the digital-to-analogue converter (Redlab, pin 30) can be switched to control the power supply of the Sharp S202S01 SSR which again is used to interrupt or establish the 230 VAC power supply to the electro-pneumatic valve. The voltages are converted using a SN74LS06N TTL-to-CMOS converter (TTL). 


\section{Appendix B: Software implementation}

A LabView program for recording the needed datasets, setting the computer-connected devices and for remote control was originally written by Heiko Huth and migrated to run with the AC calorimeter setup used for this work. The program was extended to measure additional quantities like DC voltage or heater resistance.

The main program is divided into 4 tabs. The first tab is for setting the devices lock-in amplifier, temperature controller, analog-to-digital converter and DMM (Figure 69).

The temperature control panel provides useful experimental parameters. The error and the waiting time are needed for step-like or isothermal measurements where the temperature has to stabilize. In the case shown, temperature is assumed to be stabilized and the measurement starts if the actual temperature fluctuates around the temperature set-point by less than $0.2 \mathrm{~K}$ for at least $200 \mathrm{~s}$. This feature can be disabled if needed. The Tset value gives the set-point temperature whereas the rate gives the actual heating/cooling rate in $\mathrm{K} \mathrm{h}^{-1}$.

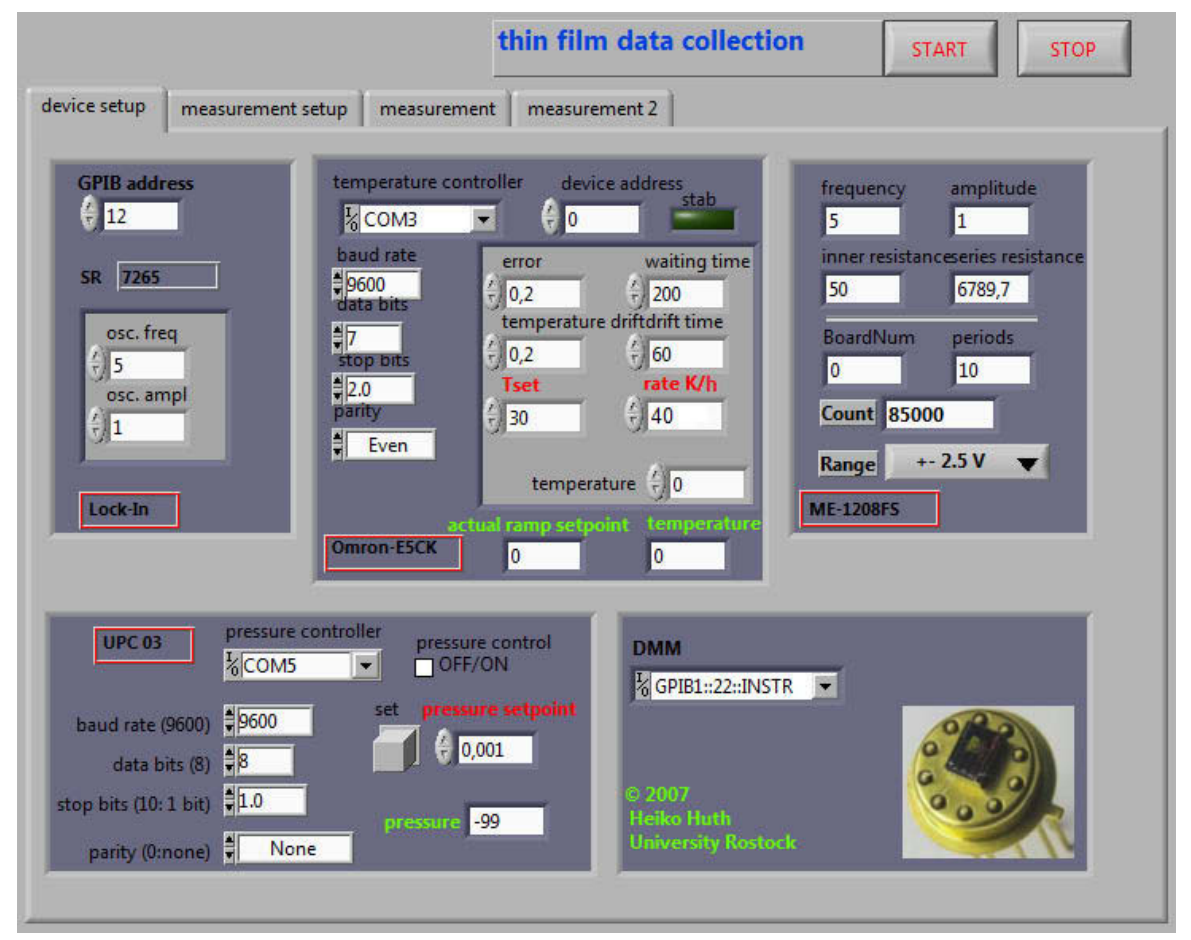

Figure 69: "Device setup" tab of the main program. This tab is for setting initial parameters for device communication, some parameters of the lock-in amplifier and for temperature control even during a measurement.

The analog-to-digital converter is used to sample the sinusoidal voltages to be measured coming from the known resistance $R_{i}$ and from the heater resistance $R_{h}$ (Figure 70) giving $U_{i}$ and $U_{h}$. From the measured data points the LabView program calculates the rms values. To get 
reliable data for the rms voltages it is necessary to average over some periods, in Figure 69 this is ten periods.

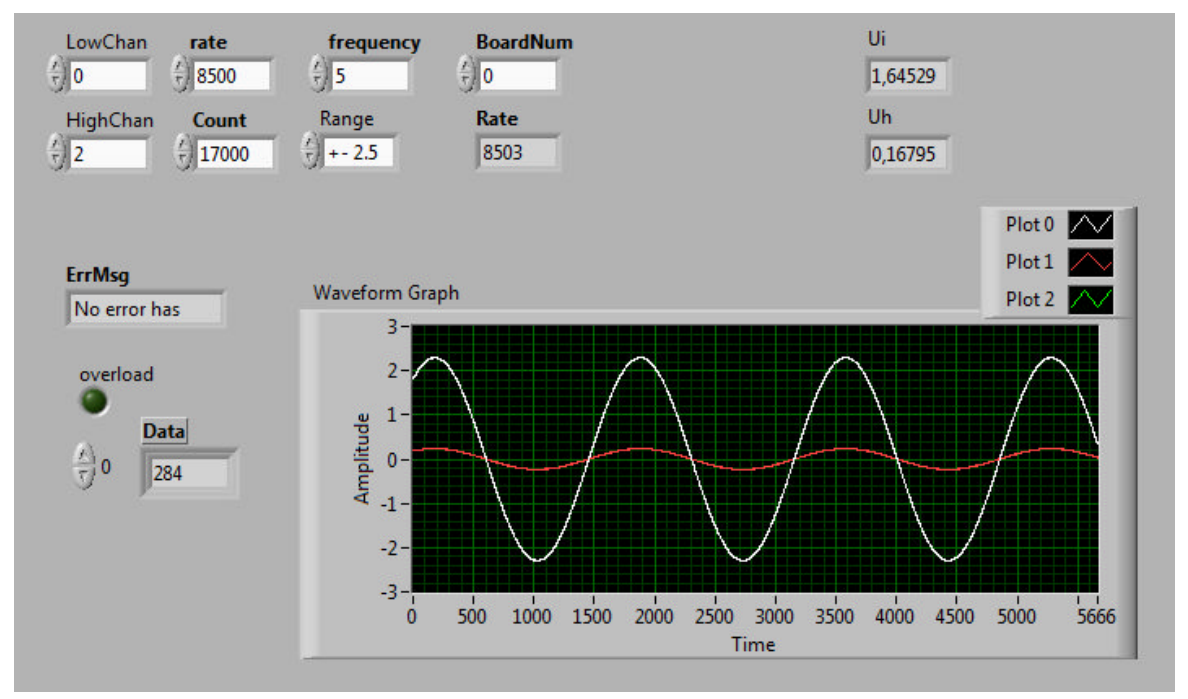

Figure 70: Sub-program for displaying the amplified sinusoidal voltages $U_{i}$ and $U_{h}$ as measured by the analog-to-digital converter. The rms voltages are calculated and displayed too.

Calculated heater resistance and power can be seen as numbers in the measurement setup tab of the main program together with the amplified voltages (Figure 71) to check if the heater resistance determination works properly. This tab is mainly for setting the temperature program by calling a sub-program. Moreover the measurement can be started and canceled in this tab as well as the data sampling can be set. 


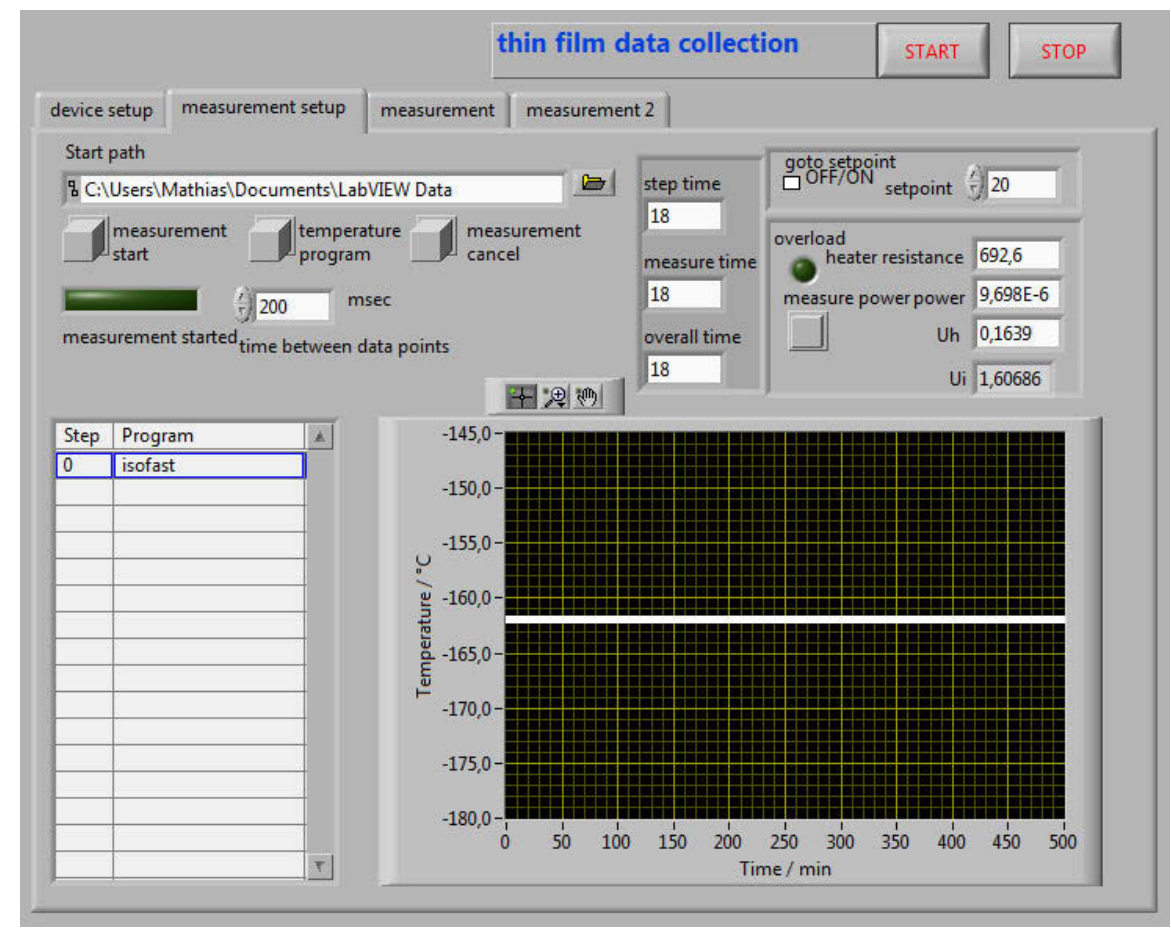

Figure 71: "Measurement setup" tab of the main program. This tab is for starting, aborting, setting and displaying the temperature program of the experiment. Calculated heater resistance and power as well as the amplified rms voltages are shown. The data sampling of the program can be set here.

The "temperature program"-button calls a sub-program for temperature program setup which gives the opportunity to select several different types of temperature program modules (Figure 72): "Temperaturescan", "goto temperature", "isotherm", "isofast" and "StepIso". The term "Temperaturescan" is self-explanatory. The "isotherm" is an isothermal program, as is also the case for the "isofast" option. "goto temperature" is again self-explanatory. The "StepIso" option is an isothermal step scan method. 


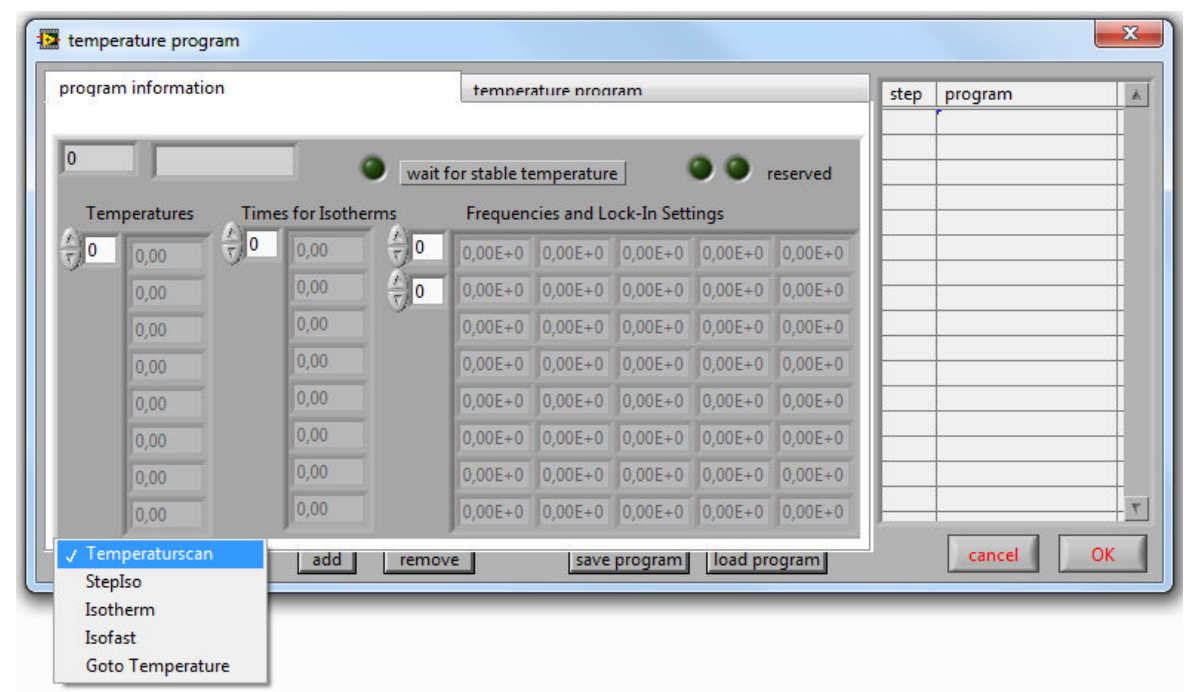

Figure 72: Sub-program for temperature program setup which pops up if the "temperature program" button of the "measurement setup" tab of the main program is pressed. The user can choose from five different modules to create a temperature profile as needed for the experiment.

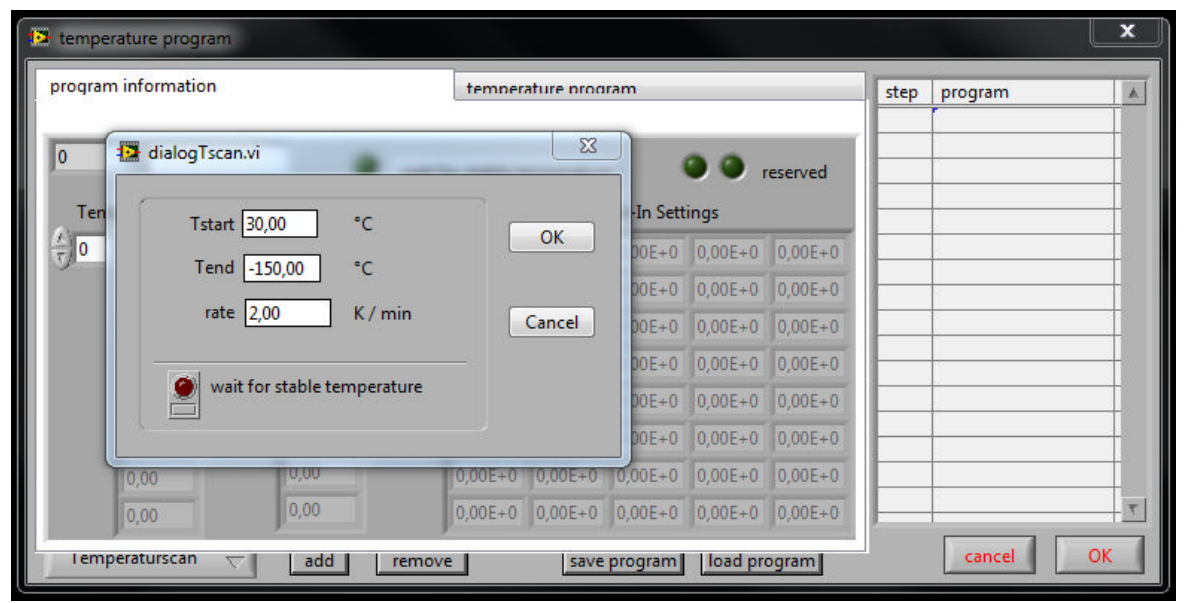

Figure 73: Pop-up in the sub-program if a temperature scan was chosen and added to the temperature profile. These parameters will be transmitted to the temperature controller.

Once a module is added to the temperature program another window opens to define the needed parameters. For example this is done for a cooling scan (Figure 73). Hereafter the needed parameters for the lock-in initialization have to be set in another window (Figure 74). This can be done now for many steps and with any of the available types of temperature program modules to 
create the temperature program as needed for a certain experiment. A cooling-heating cycle with isothermals in between looks then as is shown in Figure 75.

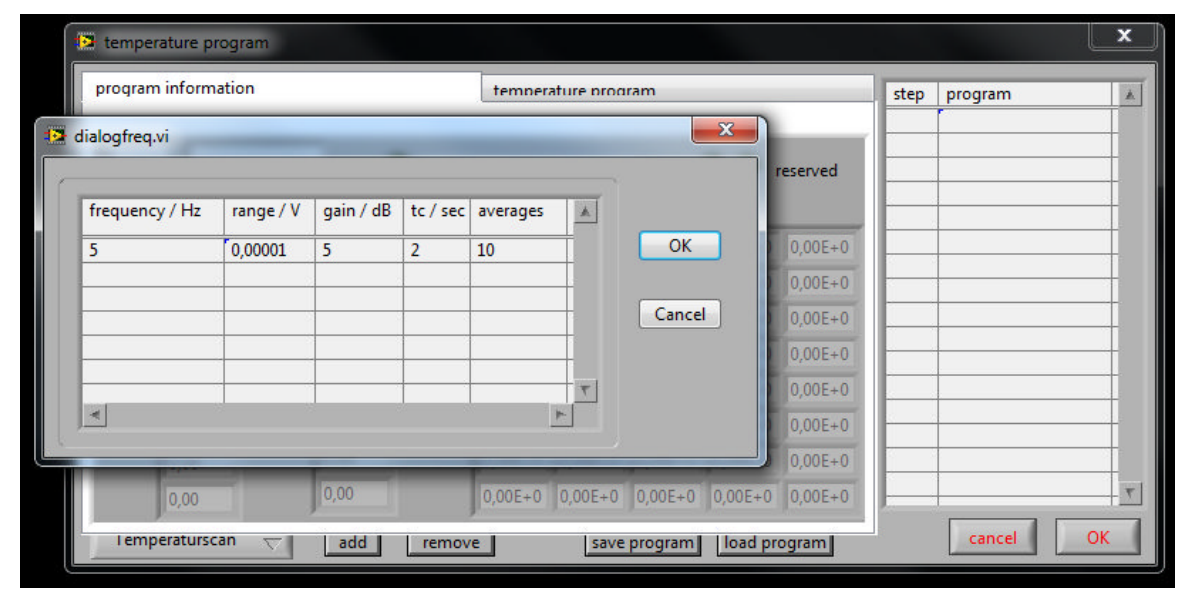

Figure 74: Pop-up window that appears to set the needed parameters for the lock-in amplifier. The amplifier will be initialized with these values at startup of each module of the final temperature program.

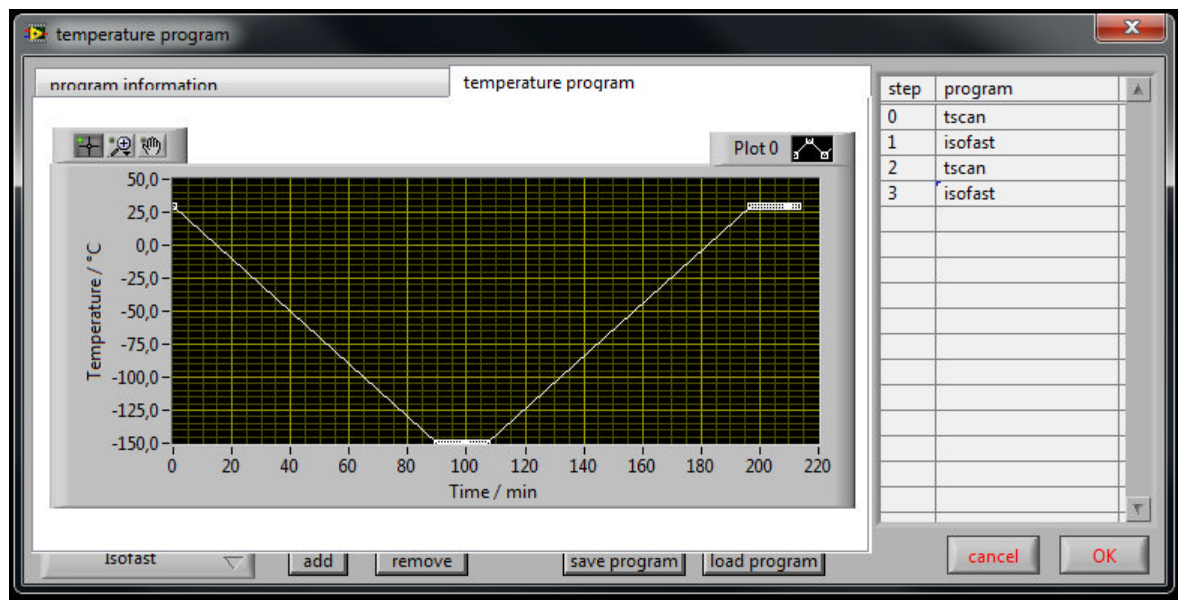

Figure 75: Example of a cooling-heating temperature profile, with each ramp followed by a $1000 \mathrm{~s}$ isotherm.

If the measurement is started the "measurement" tab of the main program displays the measured values: thermopile magnitude and phase (Figure 76). Different x-axes can be chosen such as time, temperature, frequency or heater resistance. The "measurement 2 " tab gives access to parameters needed for deposition control such as material parameters and actions like opening/closing the bi-stable shutter valve (Figure 77). The control "Thickness setpoint" defines the thickness of the film to deposit onto the sensor in terms of thermopile amplitude. When this thickness is reached the shutter valve closes automatically. 
In all, 16 measured and calculated quantities are logged by the software to an ASCII-file every data sampling period. The 16 quantities in the order they appear in the header of the file are: measured Pt100 temperature, temperature set-point, oscillator frequency, thermopile magnitude, phase, power, heater resistance, pressure (fragment of older version of the software), measure time, step time, DC voltage, shutter valve parameter (" 0 " for closed, "1" for open), thickness as measured by the QCM, deposition rate as measured by the QCM, $U_{i}$ and $U_{h}$. Together with the data file a temperature program file is saved containing the used temperature profile. Moreover another file containing some settings as done in the "device setup" tab of the main program is saved in the measurement folder

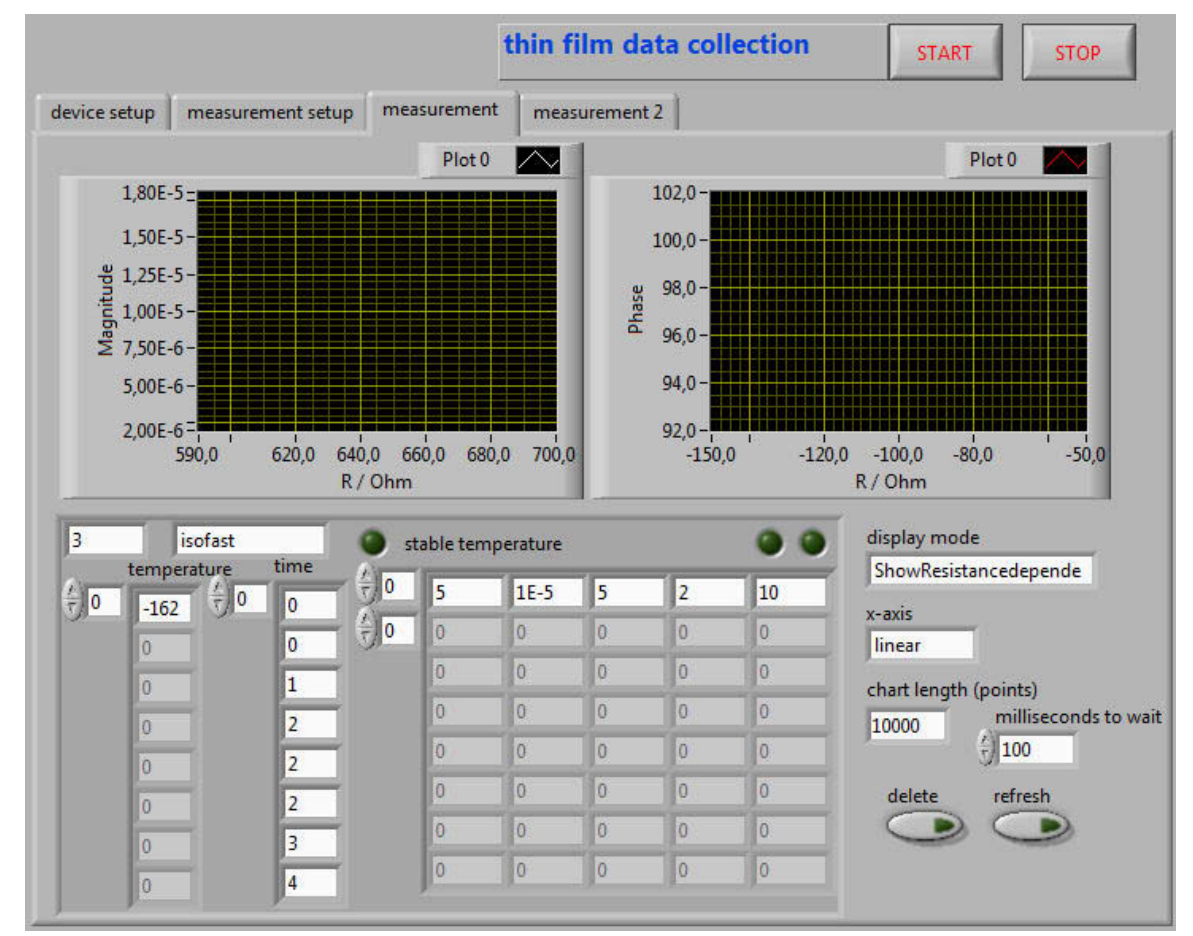

Figure 76: "Measurement" tab of the main program for displaying the measured parameters: thermopile magnitude and phase. The x-axis can be chosen to be Pt100 temperature, time, heater resistance or oscillator frequency. 


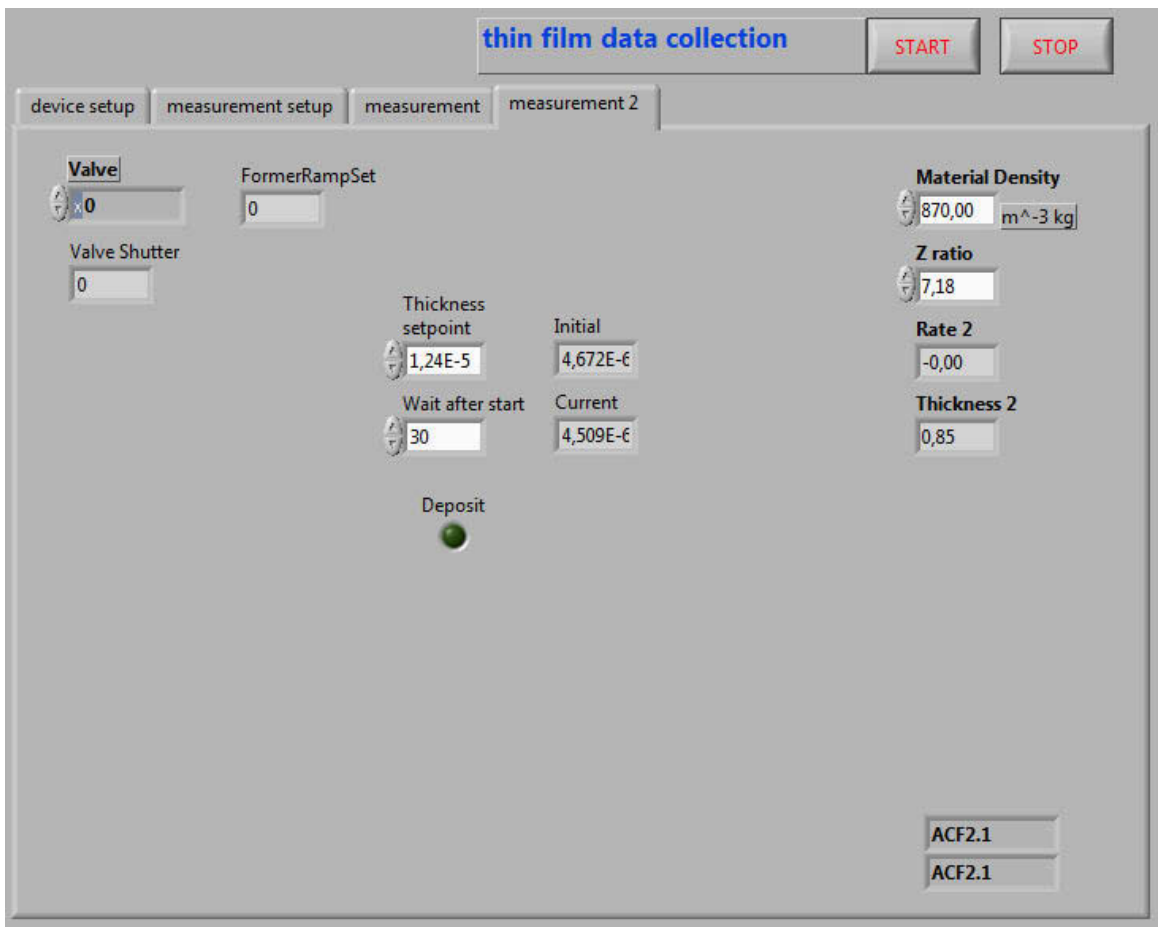

Figure 77: "Measurement 2" tab of the main program for setting some of the parameters for deposition. 


\section{Danksagung}

Während meiner Arbeit standen mir viele Freunde und Kollegen mit Rat und Tat zu Seite deren Unterstützung ich zu schätzen weiß und bei denen ich mich hiermit aufrichtig bedanken möchte. Allem voran bedanke ich mich bei Herrn Dr. H. Huth für die hilfreiche Unterstützung beim experimentellen Aufbau und bei Problemen mit dem Messsystem sowie für die immer bereitwillige Beantwortung meiner Fragen.

Diese Arbeit wurde im Rahmen eines Gemeinschaftsprojekts mit der Arbeitsgruppe um Professor Mark D. Ediger von der University of Madison WI angefertigt. Für die früchtetragenden Zusammenarbeit bedanke ich mich insbesondere bei zwei seiner Mitarbeiter, bei Ken L. Kearns und Katie R. Whitaker. Ganz besonderen Dank gilt Katie für das Korrekturlesen dieser Arbeit.

Ich danke Yeong Zen Chua für die Durchführung einiger Messungen, die diese Arbeit bereichert haben.

Ein nicht unwesentlicher Teil dieser Arbeit entstand in enger Zusammenarbeit mit Evgeni Shoifet, der das Finite Elemente Modell ausgearbeitet hat. Ohne seine Hilfe wären viele Fragen unbeantwortet geblieben.

Weiterer Dank gilt Ranko Richert und Zhen Chen, die mir Daten zur frequenzabhängigen Dynamik von Ethylbenzene geliefert haben.

Ich danke meinem Betreuer Professor Christoph Schick, der mir immer hilfreich bei den verschiedensten Fragestellungen Antwort gab, sowie Professor Mark D. Ediger, der in der Vielzahl von Telefonkonferenzen maßgeblich zum Ertrag dieses Projektes beigetragen hat.

Nicht zuletzt bedanke ich mich bei meinen Freunden und meiner Familie, die meinem Leben neben der Arbeit Erfüllung gegeben haben. Dabei gilt ganz besonderer Dank meiner Verlobten Jana, die mit ihrer positiven Einstellung und Liebe zu mir mein Leben unermesslich bereichert hat.

Mathias Ahrenberg 


\section{Acknowledgement}

During my work a lot of friends and colleagues supported me with help and advice and I appreciate their support. All of them I want to thank hereby sincerely. First of all I would like to thank Dr. H. Huth for his helpful assistance in experimental setup and in case of problems with the measurement system. He was always willing to answer my questions.

This work was done as part of a joint project with the research group led by Professor Mark D. Ediger of the University of Madison WI. For fruitful collaboration, I would like to thank especially two of his staff, namely Ken L. Kearns and Katie R. Whitaker. Very special thanks go to Katie for the proof reading.

I also thank Yeong Zen Chua for performing some measurements that have enriched this work.

A considerable part of this work was done in close cooperation with Evgeni Shoifet who has worked out the finite element model. Without his help, many questions were left unanswered.

Another thank goes to Ranko Richert and Zhen Chen, who have provided me data of the frequency dependent dynamics of ethylbenzene.

I thank my supervisor Professor Christoph Schick, who was always helpful to me to a variety of questions, and Professor Mark D. Ediger, who contributed significantly to the income of the project in numerous teleconferences.

Last but not least, I would like to thank my friends and family who have fulfilled my life besides work. Very special thanks go to my fiancée Jana, who has enriched my life immeasurably with her positive attitude and love.

Mathias Ahrenberg 


\section{Selbständigkeitserklärung}

Hiermit versichere ich, dass ich die vorliegende Arbeit selbständig und ohne fremde Hilfe verfasst habe. Andere als die von mir angegebenen Hilfsmittel und Quellen wurden nicht verwendet. Die den benutzten Werken wörtlich oder inhaltlich entnommenen Stellen sind als solche kenntlich gemacht.

Mathias Ahrenberg 